\title{
Unraveling Halogen Effects in Supramolecular Polymerization
}

\section{Jonas Matern, Nils Bäumer, Gustavo Fernández*}

*Organisch-Chemisches Institut, Universitat Münster, Corrensstraße 36, 48149 Münster, Germany

Table of Contents

Denaturation Model for cooperative supramolecular Polymerizations .................................................................2

Thermodynamic Parameters .......................................................................................................................................................

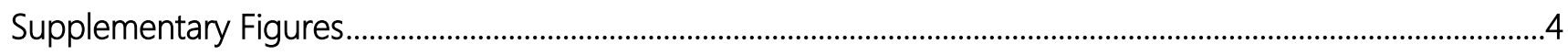

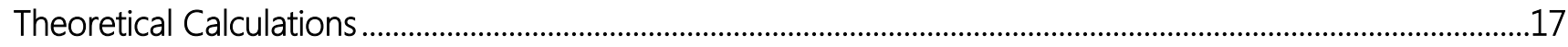

Experimental Section …………………………………………………………………………………………....

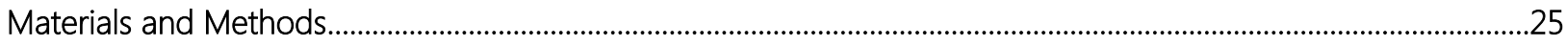

Synthesis and Characterization ...................................................................................................................................26

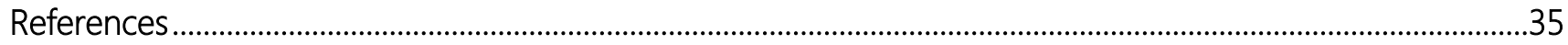




\section{Denaturation Model for cooperative supramolecular Polymerizations}

The denaturation model ${ }^{1}$ is based on the concentration-dependent supramolecular polymerization equilibrium model by Goldstein \& Stryer where the polymerization is described as a sequence of monomer addition equilibria. ${ }^{2}$

$$
\begin{aligned}
& {\left[P_{n}\right]=K_{n}\left[P_{n-1}\right][X]} \\
& {\left[P_{n+1}\right]=K_{e}\left[P_{n}\right][X]} \\
& {\left[P_{i}\right]=K_{e}\left[P_{i-1}\right][X]}
\end{aligned}
$$

For the cooperative model $\mathrm{K}_{\mathrm{n}}<\mathrm{K}_{\mathrm{e}}$ and for isodesmic process $\mathrm{K}_{\mathrm{n}}=\mathrm{K}_{\mathrm{e}}$. The concentration for each species $P_{\mathrm{i}}$ is given by $\left[P_{i}\right]=$ $K_{n}^{i-1}[X]^{i}$ for $i \leq n$ and $\left[P_{i}\right]=K_{e}^{i-n} K_{n}^{n-1}[X]^{i}$ for $i>n$.

The dimensionless mass balance is obtained by inserting the dimensionless concentration $p_{i}=K_{e}\left[P_{i}\right]$, the monomer concentration $x=K_{e}[X]$ and the concentration of each species $P_{i}($ for $i \leq n): p_{i}=\sigma^{i-1} x^{i}$ and for $\left.i>n: p_{i}=\sigma^{n-1} x^{i}\right)$ :

$$
x_{\text {tot }}=\sigma^{-1} \sum_{i=1}^{n} i(\sigma x)^{i}+\sigma^{n-i} \sum_{i=n+1}^{\infty} i x^{i}
$$

Both sums are evaluated by using standard expressions for converging series:

$$
x_{t o t}=\left(\frac{(\sigma x)^{n+1}(n \sigma x-n-1)}{(\sigma x-1)^{2}}+\frac{\sigma x}{(\sigma x-1)^{2}}\right)-\sigma^{n-1}\left(\frac{x^{n+1}(n x-n-1)}{(x-1)^{2}}\right)
$$

With $x_{\text {tot }}=c_{\text {tot }} K_{e}$ and $\mathrm{c}_{\mathrm{tot}}$ : total monomer concentration.

The sum solved by standard numerical methods (Matlabfzerosolver) yields the dimensionless monomer concentration $x$. Considering that every species with $i>1$ is defined as aggregate, the degree of aggregation results in:

$$
\alpha A g g=\frac{x_{t o t}-x}{x_{t o t}}
$$

Via $K_{e}=\exp \left(-\frac{\Delta G^{0}}{R T}\right)$, the denaturation curves can be obtained with $f$ defined as volume fraction of good solvent:

$$
\Delta G^{0,}=\Delta G^{0}+m f
$$

It is assumed that the cooperativity factor $\sigma$ is independent on the volume fraction and the $m$ value for the elongation regime equals the $m$ value for nucleation.

The denaturation data needs to be transformed into the normalized degree of aggregation, if fitted to the supramolecular polymerization equilibrium model:

$$
(f)=\frac{A(f)-A(f=0)}{A(f=1)-A(f=0)}
$$


The optimization of the four needed parameters $\left(\Delta G_{0}, m, \sigma\right.$ and $\left.p\right)$ to fit the equilibrium model to the experimental data (normalized degree vs. $f$ ) is done by the non-linear least-squares analysis using Matlab (lsqnonlinsolver). The data is then fitted with the non-linear least squared regression (Levenberg-Marquardt algorithm). 
Table S1. Summary of the thermodynamic parameters of the different aggregates of complexes 1 - $\mathbf{3}$.

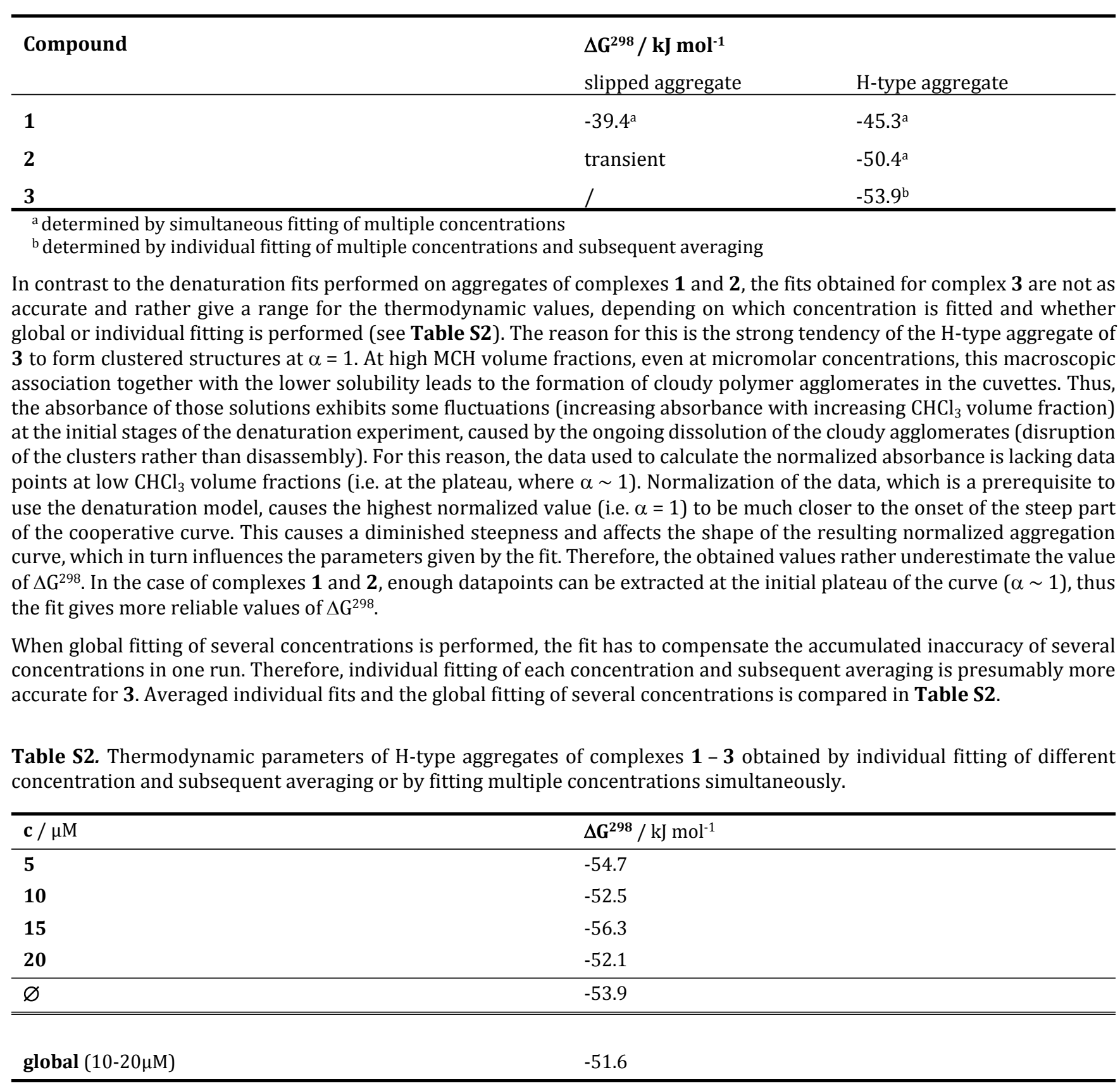

The correctness of the postulated trend in stability of the H-type assemblies $(\mathbf{3}>\mathbf{2}>\mathbf{1})$, could furthermore be confirmed by arbitrarily fixing the value of the m-parameter, which affects the steepness and onset of the curve, before fitting the data for the H-type aggregates of all three complexes. In any case, the obtained Gibb's free energies of $\mathbf{3}$ were lower than the ones of $\mathbf{1}$ and $\mathbf{2}$, in accordance with the postulated seesaw-like energy landscape. 


\section{Supplementary Figures}
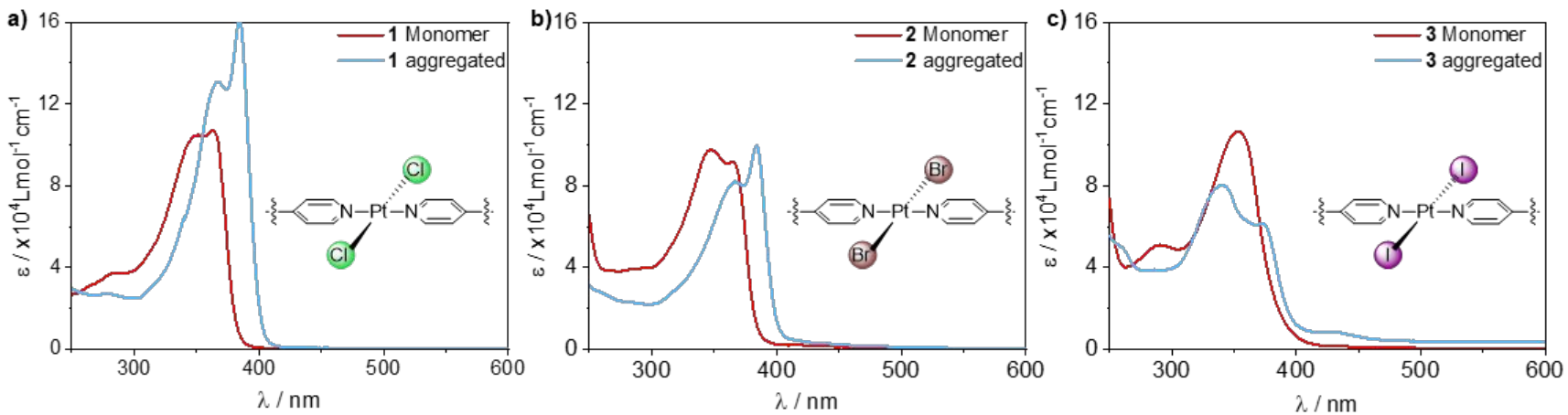

Figure S1. UV/Vis absorption spectra of complexes of $\mathbf{1}(\mathrm{a}), \mathbf{2}(\mathrm{b})$ and $\mathbf{3}$ (c) in a good solvent $\left(\mathrm{red}, \mathrm{CHCl}_{3}\right)$ and an aggregation-inducing "bad" solvent (blue, $\mathrm{MCH}$ for $\mathbf{1}+2,9-1 \mathrm{MCH}-\mathrm{CHCl}_{3}$ for 3; c $=10 \mu \mathrm{M}, \mathrm{T}=298 \mathrm{~K}$ ).
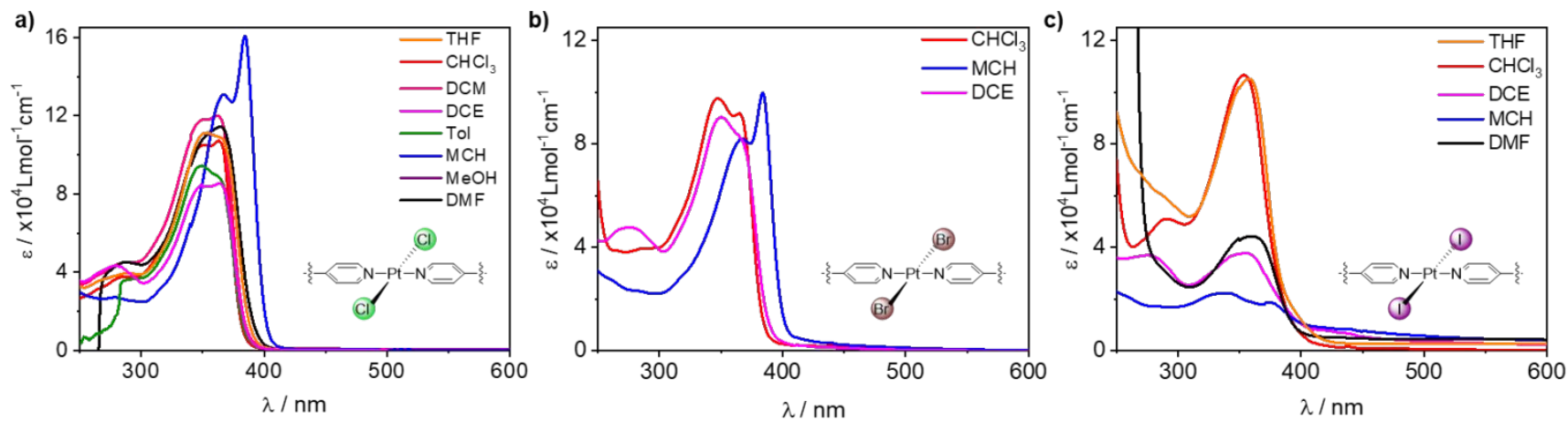

Figure S2. UV/Vis spectra of $\mathbf{1}$ (a), 2 (b) and 3 (c) in different solvents (c = $10 \mu \mathrm{M}, T=298 \mathrm{~K}$ ). All three compounds are molecularly dissolved in good solvents such as DCM, $\mathrm{CHCl}_{3}$ or THF, where the main absorption band is centered around $350 \mathrm{~nm}$ Notably, $\mathbf{1}$ and 2 both exhibit a splitting of the monomer absorption band with maxima at $\lambda=348 \mathrm{~nm}$ and $364 \mathrm{~nm}$ whereas the absorption of 3 is broader, with a single maximum at $\lambda=354 \mathrm{~nm}$. Although this broadening and loss of fine structure could be an indicator of the onset of self-assembly, the presence of monomers could be corroborated by means of FT-IR and NMR spectroscopy (vide infra, Figure S3).
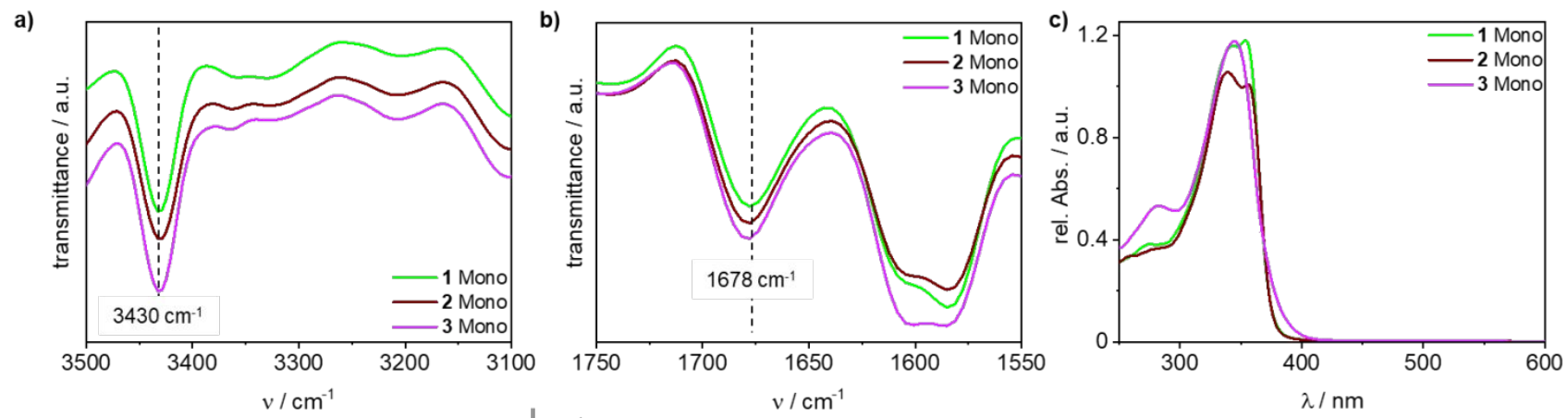

d)

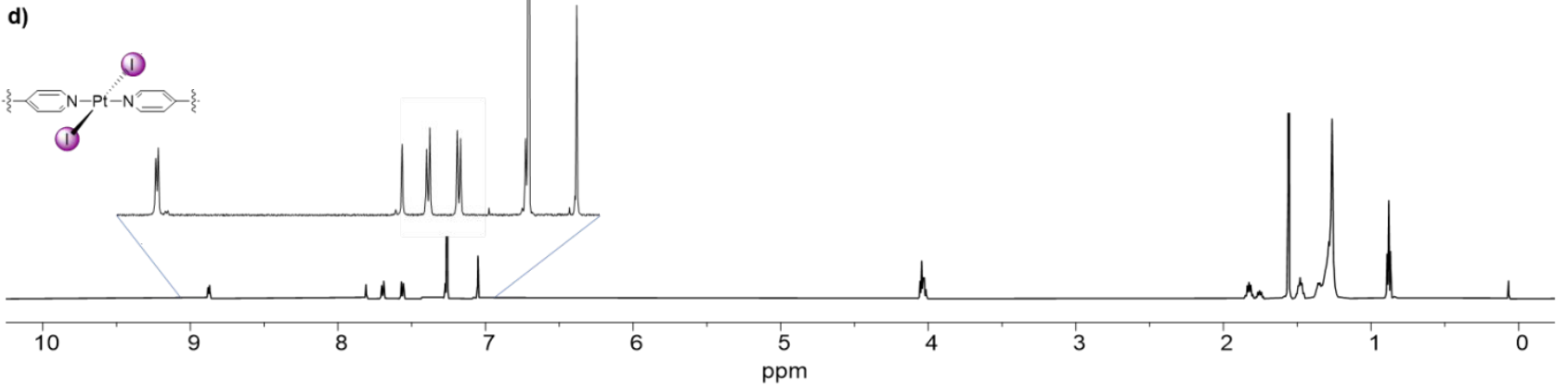

Figure S3. FT-IR- (a,b) and corresponding UV/Vis spectra (c) of solutions of $\mathbf{1}, \mathbf{2}$ and $\mathbf{3}$ in $\mathrm{CHCl}_{3}(\mathrm{c}=1 \mathrm{mM}, T=298 \mathrm{~K})$. The wavenumbers observed for the amide $\mathrm{N}-\mathrm{H}$ stretching $\left(3430 \mathrm{~cm}^{-1}\right)$ and carbonyl $\mathrm{C}=0$ stretching $\left(1678 \mathrm{~cm}^{-1}\right)$ are typical for free, non-hydrogen bonded amide groups, confirming the observation of the monomer state in the UV/Vis spectra. d) ${ }^{1} \mathrm{H}-\mathrm{NMR}$ spectrum of 3 in $\mathrm{CDCl}_{3}(\mathrm{c}=1 \mathrm{mM}, T=298 \mathrm{~K})$, showing wellresolved signals without any broadening effects that would indicate polymerization. 

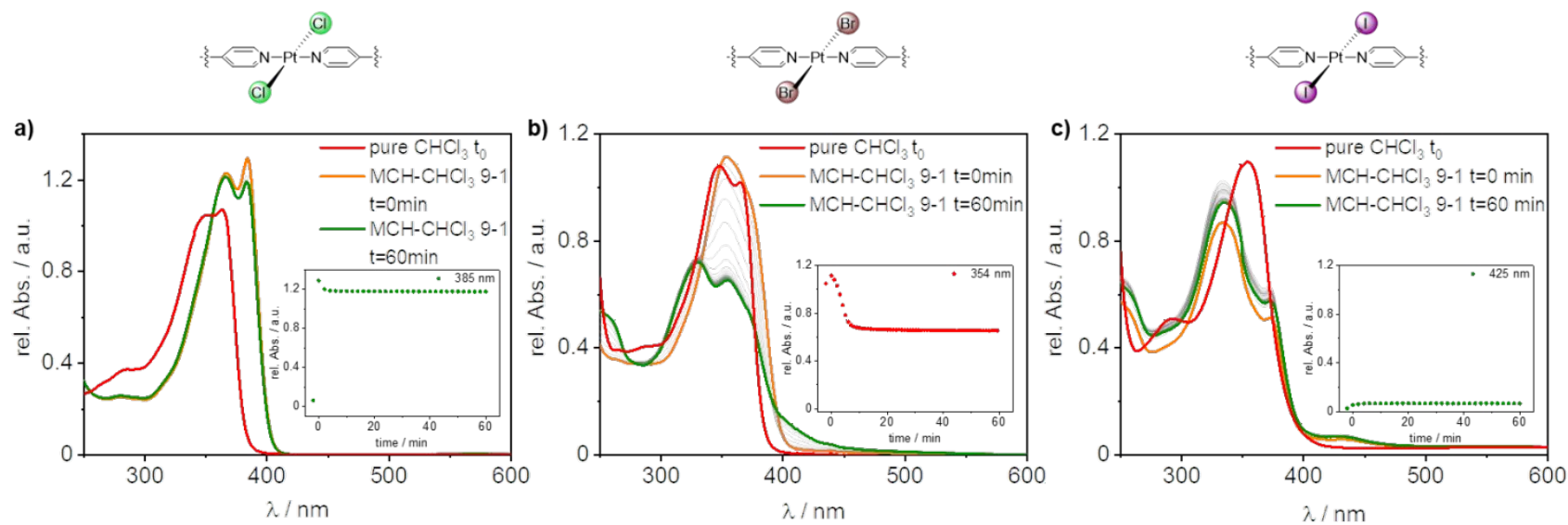

Figure S4. Time-dependent evolution of solutions of $\mathbf{1}$ (a), $\mathbf{2}$ (b) and $\mathbf{3}$ (c) after addition of nine aliquots of MCH to a solution of the respective complex in one aliquot of $\mathrm{CHCl}_{3}$ (final composition $\mathrm{MCH}-\mathrm{CHCl}_{3} 9-1, \mathrm{c}=10 \mu \mathrm{M}$ ). 


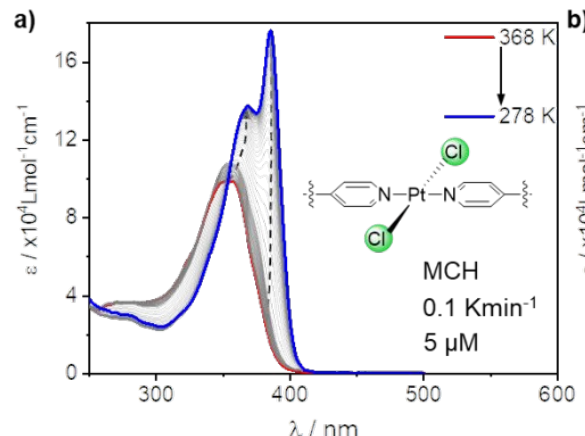

d)

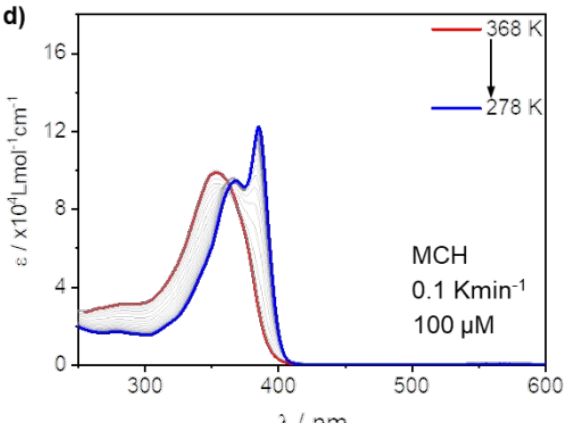

f)

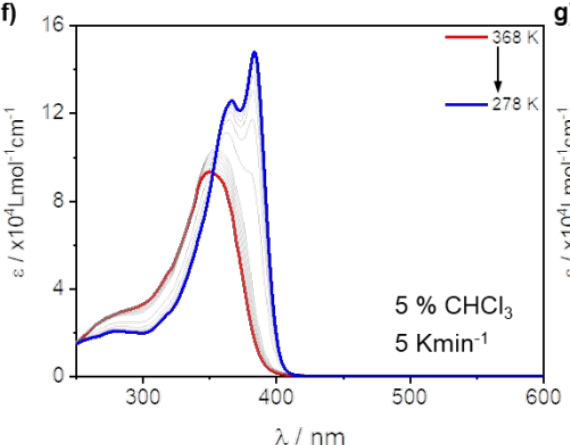

i)
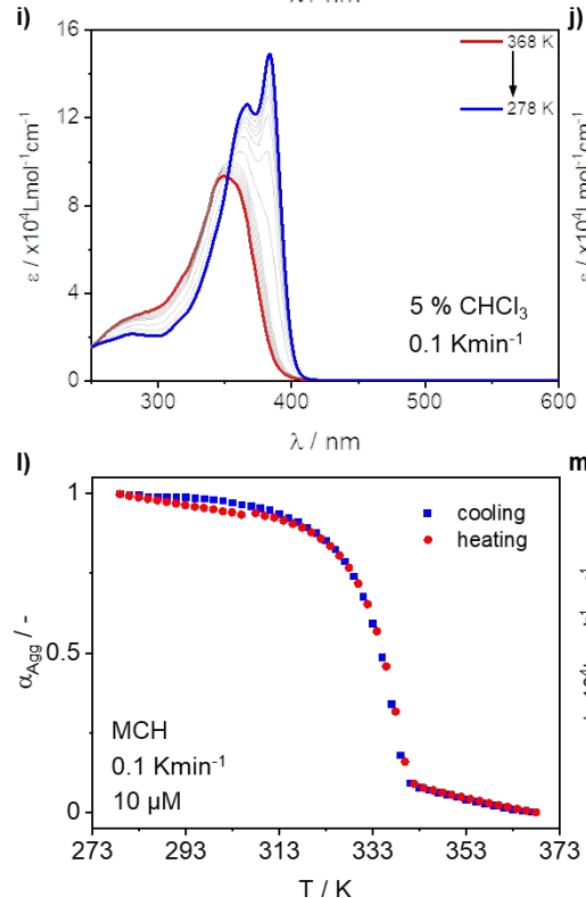
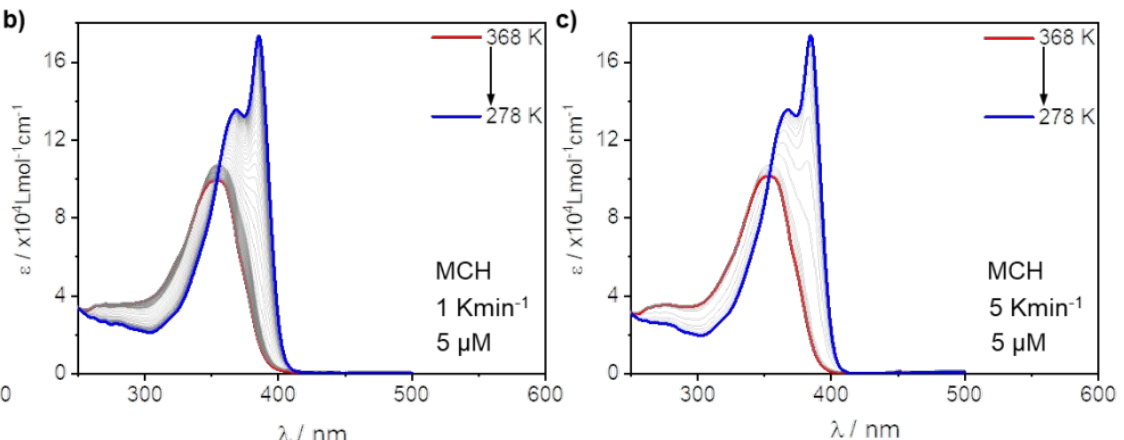

e)
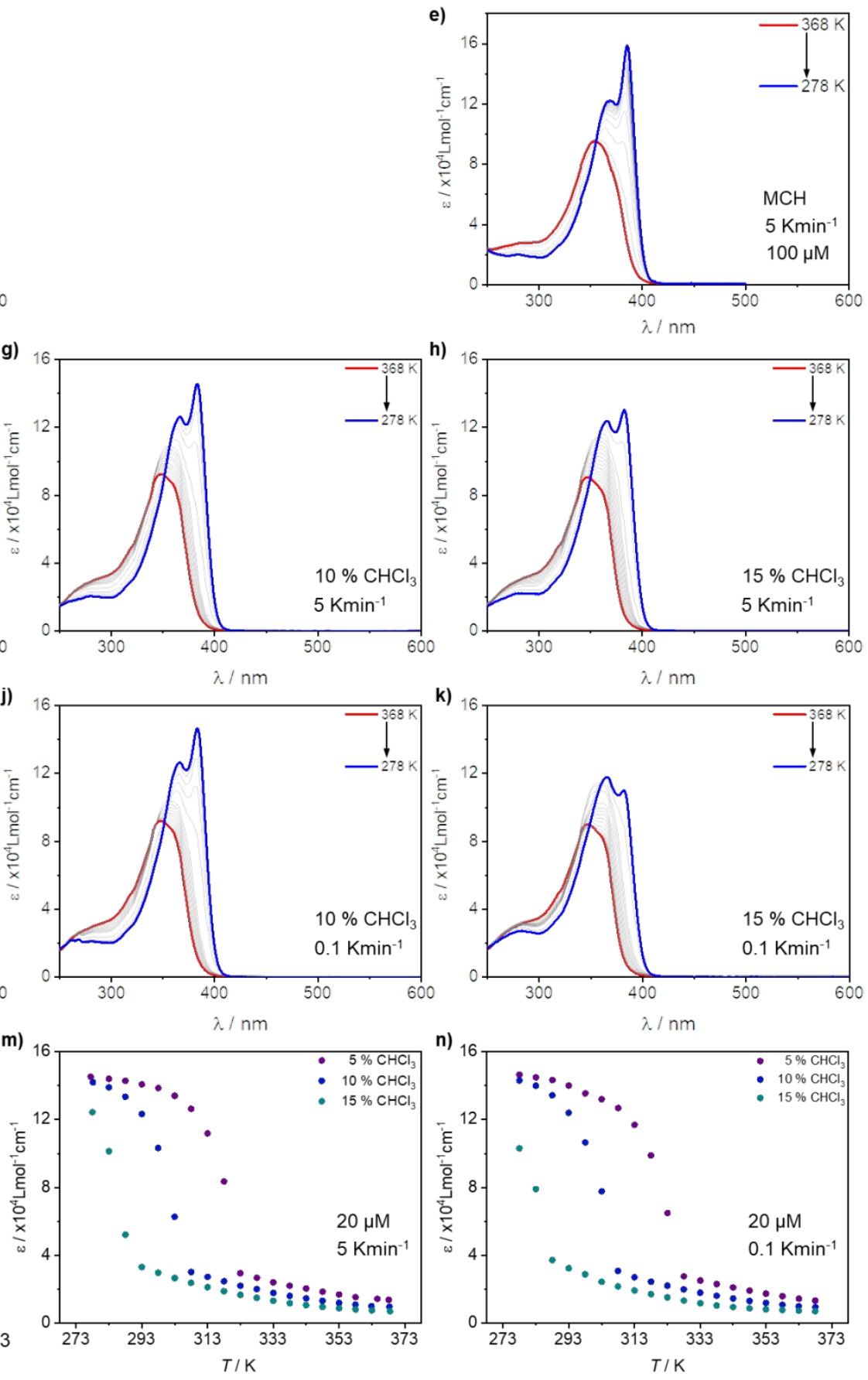

Figure S5. VT-UV/Vis studies of the supramolecular polymerization of 1 at different cooling rates, concentrations and solvent compositions: a-c) Spectra recorded at low concentration $(c=5 \mu \mathrm{M})$ and $d+e)$ at high concentration $(c=100 \mu \mathrm{M})$ at different rates. f-k) Effect of increasing volume fractions of good solvent in $\mathrm{MCH}\left(5-15 \% \mathrm{CHCl}_{3}, \mathrm{c}=20 \mu \mathrm{M}\right)$ : f-h) Spectra recorded at a rate of $5 \mathrm{Kmin}^{-1}$. i-k) Spectra recorded at a rate of $0.1 \mathrm{Kmin}^{-1}$. l) Superimposed aggregation curves obtained in a cooling (blue) and subsequent heating cycle (red) at a rate of $0.1 \mathrm{Kmin}^{-1}$, showing no hysteresis (MCH, $c=10 \mu \mathrm{M}, \lambda=385 \mathrm{~nm}$ ). $\mathrm{m}+\mathrm{n}$ ) Development of the extinction coefficient vs. temperature for different $\mathrm{CHCl}_{3}$ volume fractions derived from the experiments shown in $\mathrm{f}-\mathrm{h}\left(5 \mathrm{Kmin}^{-1}, \mathrm{~m}\right)$ and i-k $\left(0.1 \mathrm{Kmin}^{-1}, \mathrm{n}\right)$ at $385 \mathrm{~nm}$. 

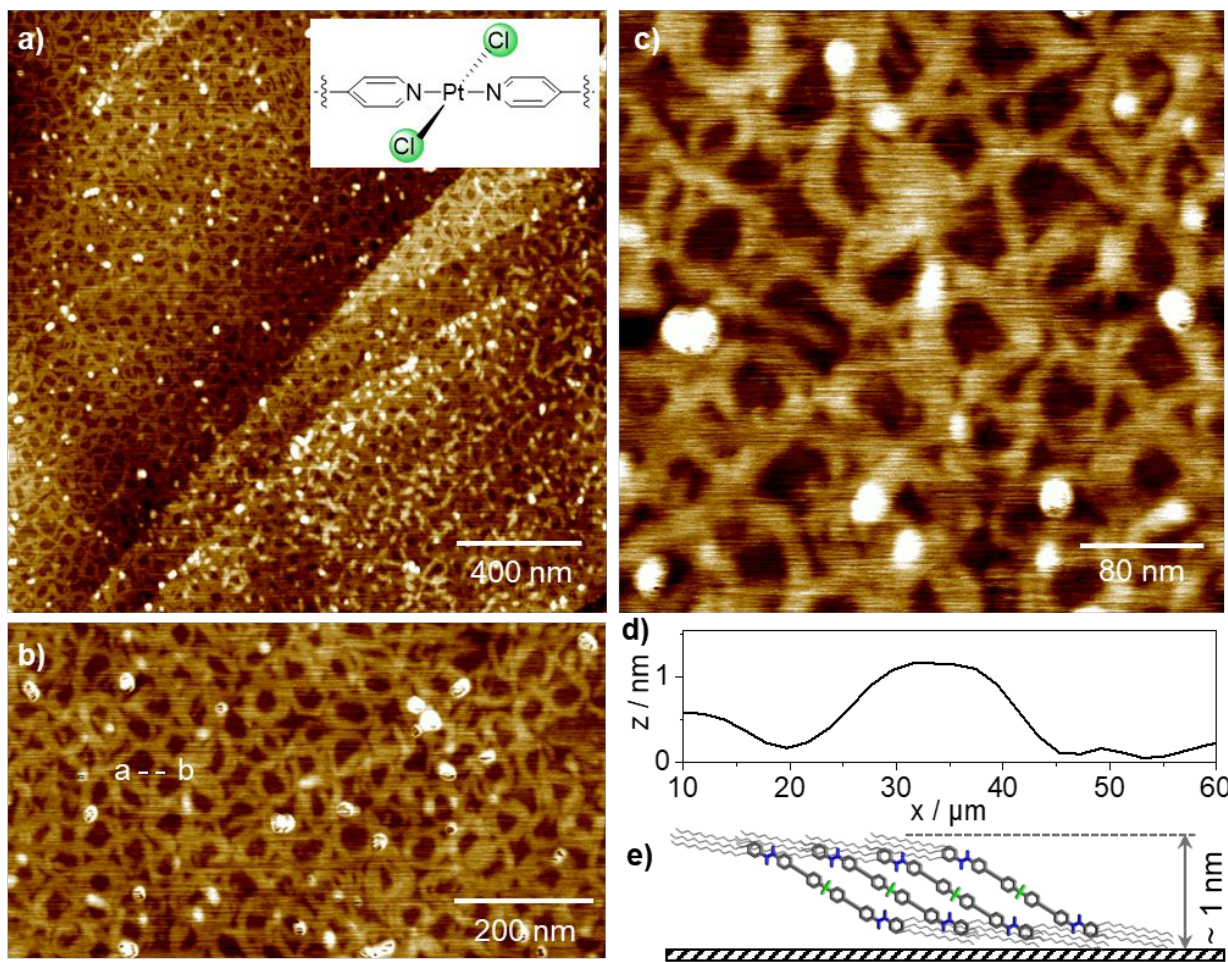

Figure S6. Morphology of slipped aggregates of 1. a-c) AFM images recorded at different levels of magnification (c $=10 \mu \mathrm{M}$, drop casted onto HOPG). d) Height profile along the a-b axis in image (b). The height of the ring structures is approximately $1 \mathrm{~nm}$. This height can be correlated to the arrangement of the polymer stacks depicted in (e), where the alkoxy-chains wrap around the exterior of the structures, parallel to the HOPG surface, maximizing vdW-interactions (e).
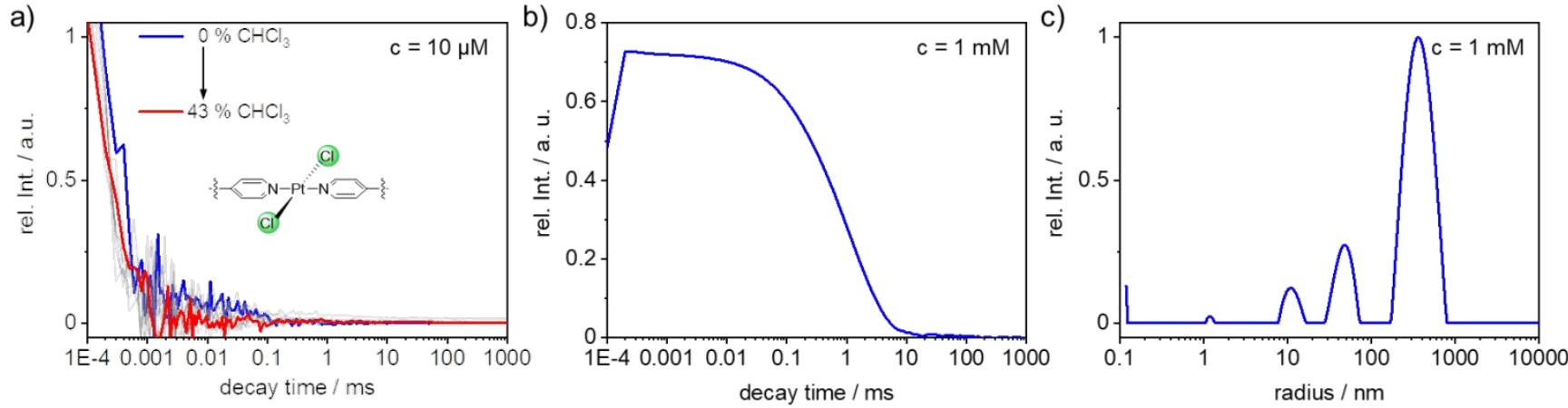

Figure S7. DLS experiments of slipped aggregate nanorings of 1. a) DLS correlation functions obtained in denaturation studies of slipped aggregates of $\mathbf{1}$ (sequential addition of aliquots of monomers dissolved in $\mathrm{CHCl}_{3}$ to the slipped aggregates in $\left.\mathrm{MCH}, \mathrm{c}=10 \mu \mathrm{M}\right)$. $\mathrm{b}+\mathrm{c}$ ) DLS correlation function (a) and size distribution (b) of nanorings of $\mathbf{1}$ obtained in $\mathrm{MCH}$ at higher concentration $(\mathrm{c}=1 \mathrm{mM})$. Considering the higher concentration at which secondary nucleation events, catenation etc. as well as the formation of larger rings is more probable, the size distributions capture the aggregate morphology in a qualitative way, although the main peak shows slightly larger size than those observed in AFM studies. 

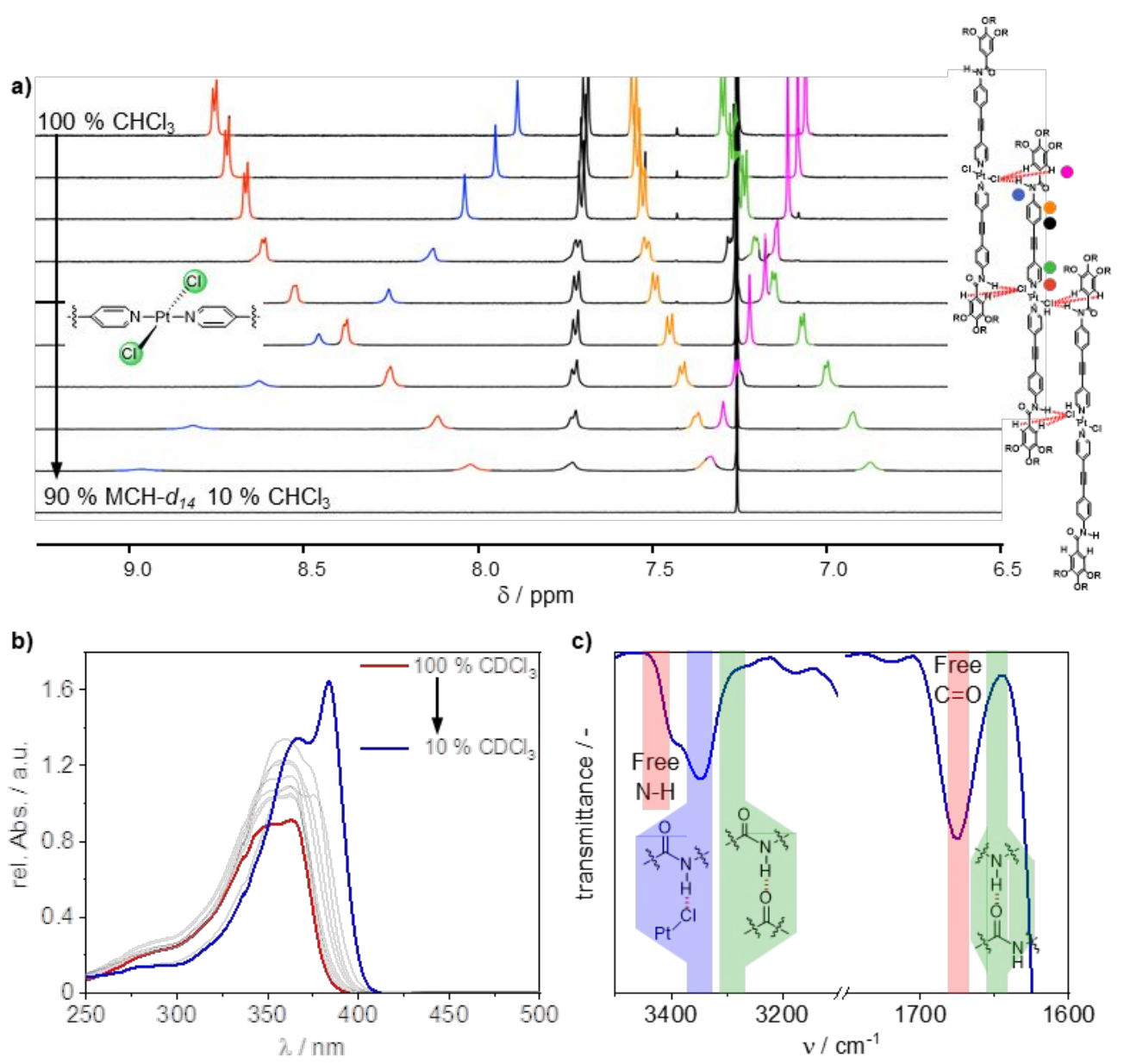

Figure S8. Packing mode studies of $\mathbf{1}$ : a) ${ }^{1} \mathrm{H}-\mathrm{NMR}$ spectra of $\mathbf{1}$ in the aromatic region, upon variation of the solvent composition (c $\left.=1 \mathrm{mM}, \mathrm{T}=298 \mathrm{~K}\right)$ and slipped molecular packing illustrating the proton assignments. b) Corresponding UV/Vis spectra showing the self-assembly into slipped aggregates. c) Thin-film FT-IR spectra of $\mathbf{1}$ in the $\mathrm{N}-\mathrm{H}-$ and $\mathrm{C}=\mathrm{O}$ stretching regions.

The self-assembly is associated with a loss of fine structure and the broadening of all shown resonances. The amide $\mathrm{N}-\mathrm{H}$ proton (blue) and the aromatic proton of the solubilizing moiety (pink) are both deshielded, implying their participation in hydrogen-bonding interactions. On the contrary, the majority of the other aromatic resonances is shifting to higher fields, diagnostic for aromatic interactions. These observations substantiate the formation of slipped stacks which are primarily stabilized by $\mathrm{N}-\mathrm{H} \cdots \mathrm{Cl}$ hydrogen bonds and aromatic interactions. The FT-IR spectra of the N-H- and carbonyl stretching region corroborate the proposed interaction pattern: The $\mathrm{N}-\mathrm{H}$ stretching frequency is shifted to slightly lower wavenumbers when compared with free amide groups. The value of $v_{\mathrm{N}-\mathrm{H}}=3348 \mathrm{~cm}^{-1}$, is characteristic for $\mathrm{N}-\mathrm{H} \cdots \mathrm{Cl}$ interactions. ${ }^{3,4} \mathrm{The} \mathrm{C}=\mathrm{O}$ stretching frequency, on the other hand, is almost unaffected $\left(v=1676 \mathrm{~cm}^{-1}\right)$.

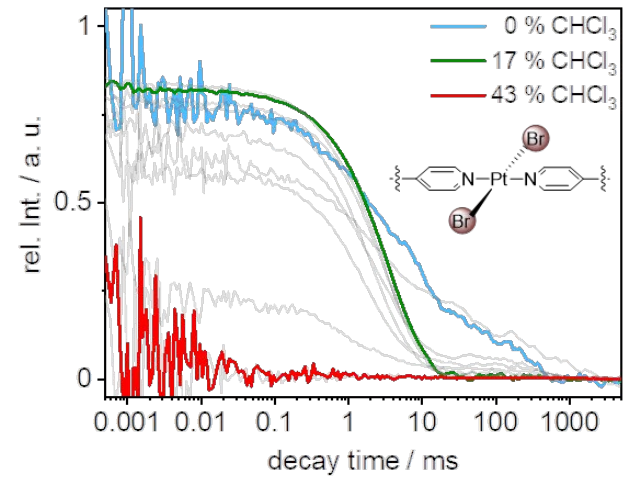

Figure S9. DLS correlation functions obtained in denaturation studies of Slipped aggregates of 2 (sequential addition of aliquots of monomers dissolved in $\mathrm{CHCl}_{3}$ to the slipped aggregate in $\mathrm{MCH}(\mathrm{c}=10 \mu \mathrm{M})$. The ill-defined correlation function in the DLS measurements of the initial solution of the denaturation studies (Figure S9), reflects the concomitant existence of a minor amount of bigger H-type SPs besides the dominant fraction of small slipped SPs. Upon increasing the $\mathrm{CHCl}_{3}$ volume fraction, the fraction of H-type SPs, which are well detected by DLS, increases, expressed through a sharpening of the correlation function. Exceeding the critical good-solvent volume fraction of $\sim 17$ vol\%, disassembly of those H-type SPs starts, resulting in more and more diffuse correlation functions. 

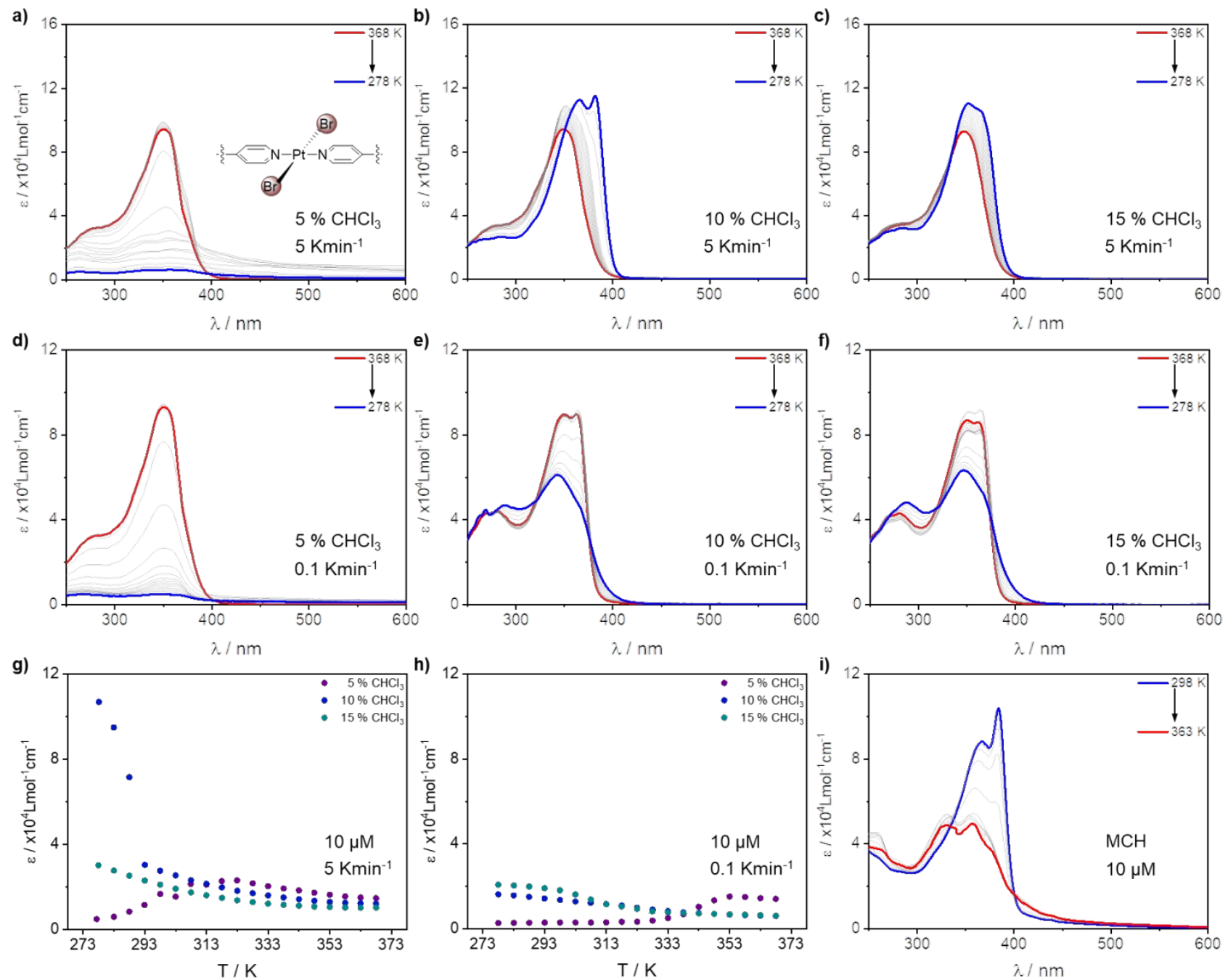

Figure S10. VT-UV/Vis studies of the supramolecular polymerization of 2 at different cooling rates and solvent compositions: a-f) Effect of increasing volume fractions of good solvent in $\mathrm{MCH}\left(5-15 \% \mathrm{CHCl}_{3}, \mathrm{c}=10 \mu \mathrm{M}\right)$ : a-c) Spectra recorded at a rate of $5 \mathrm{Kmin}^{-1}$. d-f) Spectra recorded at a rate of $0.1 \mathrm{Kmin}^{-1}$. g+h) Development of the extinction coefficient vs. temperature for different $\mathrm{CHCl}_{3}$ volume fractions derived from the experiments shown in a-c $\left(5 \mathrm{Kmin}^{-1}, \mathrm{~m}\right)$ and d-f $\left(0.1 \mathrm{Kmin}^{-1}, \mathrm{n}\right)$ at $385 \mathrm{~nm}$. i) Spectra recorded upon heating freshly prepared slipped aggregates of $2(\mathrm{c}=10 \mu \mathrm{M}, \mathrm{MCH}$, $5 \mathrm{Kmin}^{-1}$ ). Instead of a disassembly into to the monomer, the second aggregate species is formed at high temperatures, which precipitates subsequently, indicating a high thermal stability of this species in pure $\mathrm{MCH}$.

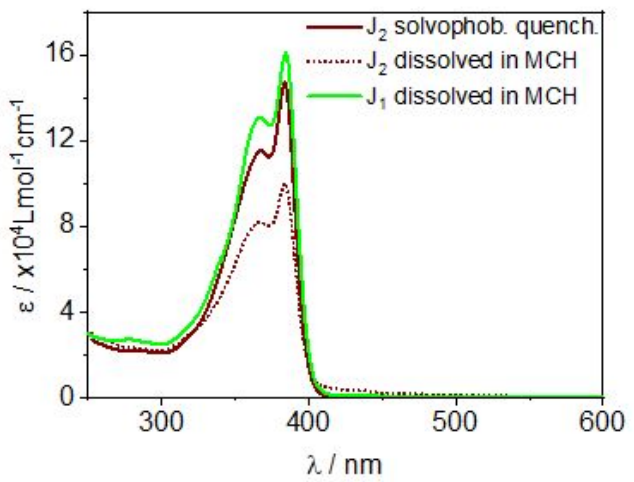

Figure S11. Comparison of the absorbance spectra of solvophobically quenched $\mathbf{2}$ (brown solid line) and the solution of $\mathbf{2}$ upon freshly dissolving the complex in MCH. The green spectrum corresponds to the slipped aggregates of $\mathbf{1}$ as a reference. It can be observed that the extinction coefficient of the slipped aggregate obtained through the quenching approach is significantly higher than the one obtained by simply dissolving solid $\mathbf{2}$ in MCH. Furthermore, the slight tailing between $400-450 \mathrm{~nm}$ in the case of non-quenched $\mathbf{2}$ (contribution of minor fraction of H-type aggregates) vanishes upon quenching. The small discrepancy to the extinction coefficients of the slipped aggregates of $\mathbf{1}$ can be explained in terms of slight deviations in the concentrations of the two solutions. 

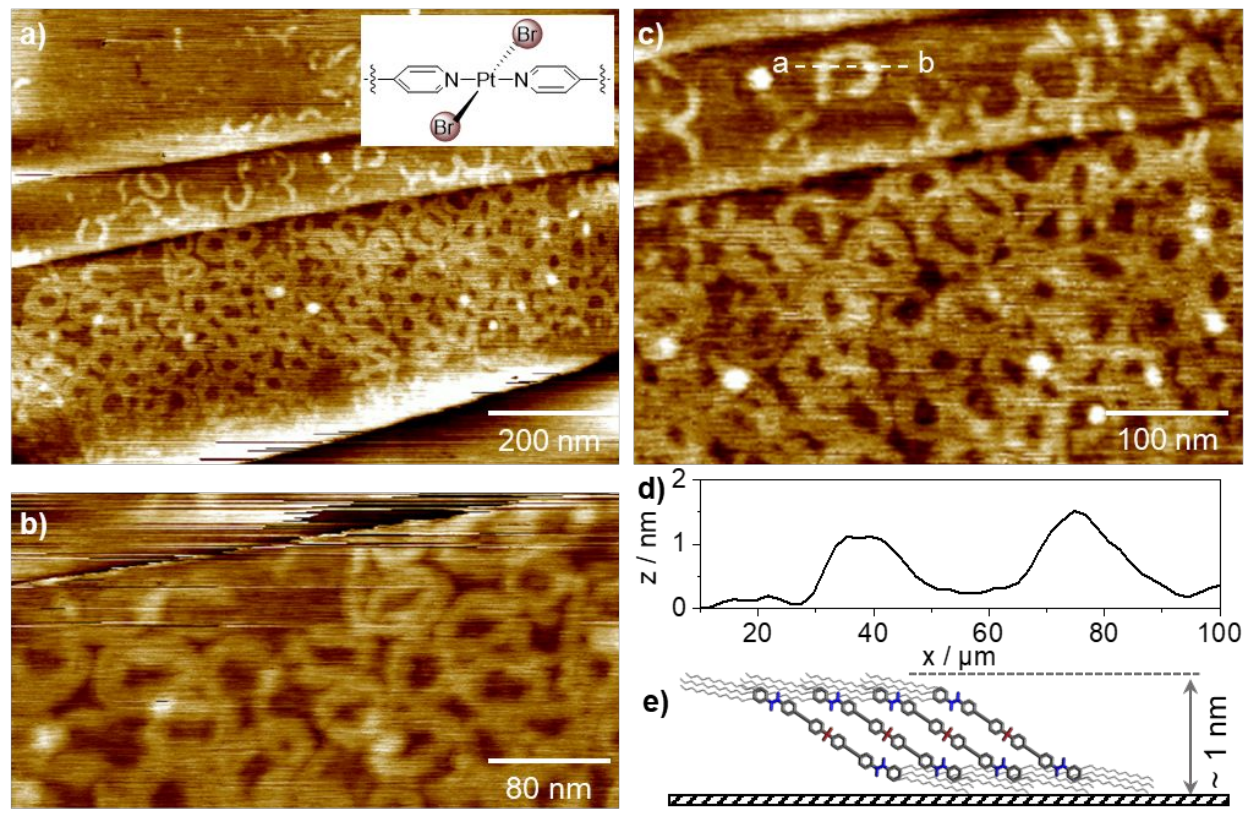

Figure S12. Morphology of slipped aggregates of 2. a-c) AFM images recorded at different levels of magnification (freshly quenched, $c=10 \mu M$, drop casted onto HOPG). d) Height profile along the a-b axis in image (c). The height of the ring structures is approximately $1 \mathrm{~nm}$. This height can be correlated to the arrangement of the polymer stacks depicted in (e), where the alkoxy-chains wrap around the exterior of the structures, parallel to the HOPG surface, maximizing vdW-interactions (e).
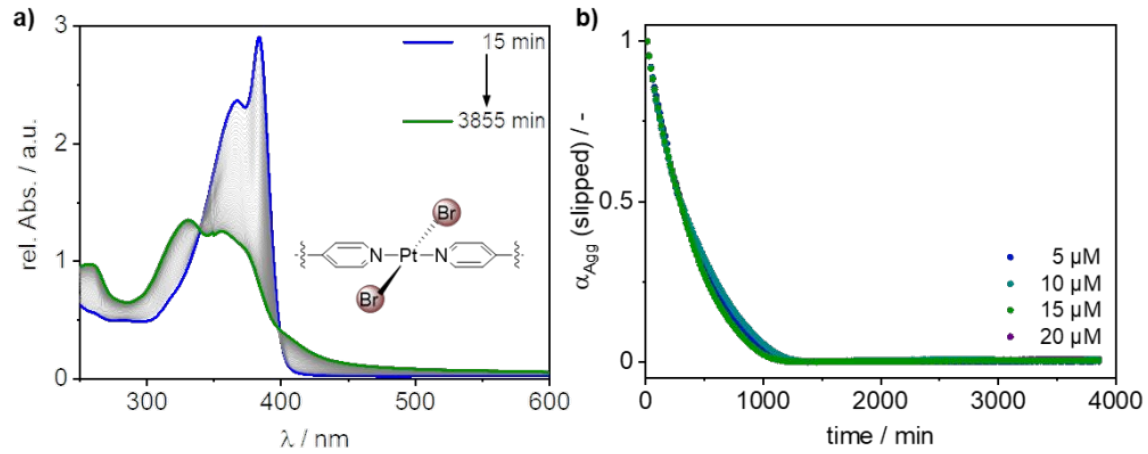

Figure S13. a) Time-resolved UV/Vis spectra showing slipped- to-H-aggregate transformation of 2 (c $=20 \mu \mathrm{M}, T=298 \mathrm{~K})$. b) Normalized absorbance at $385 \mathrm{~nm}$ plotted against time. The missing initial lag phase and the insignificant effect of concentration on the transformation speed are proof to the transient character of slipped aggregates of $\mathbf{2}$. 

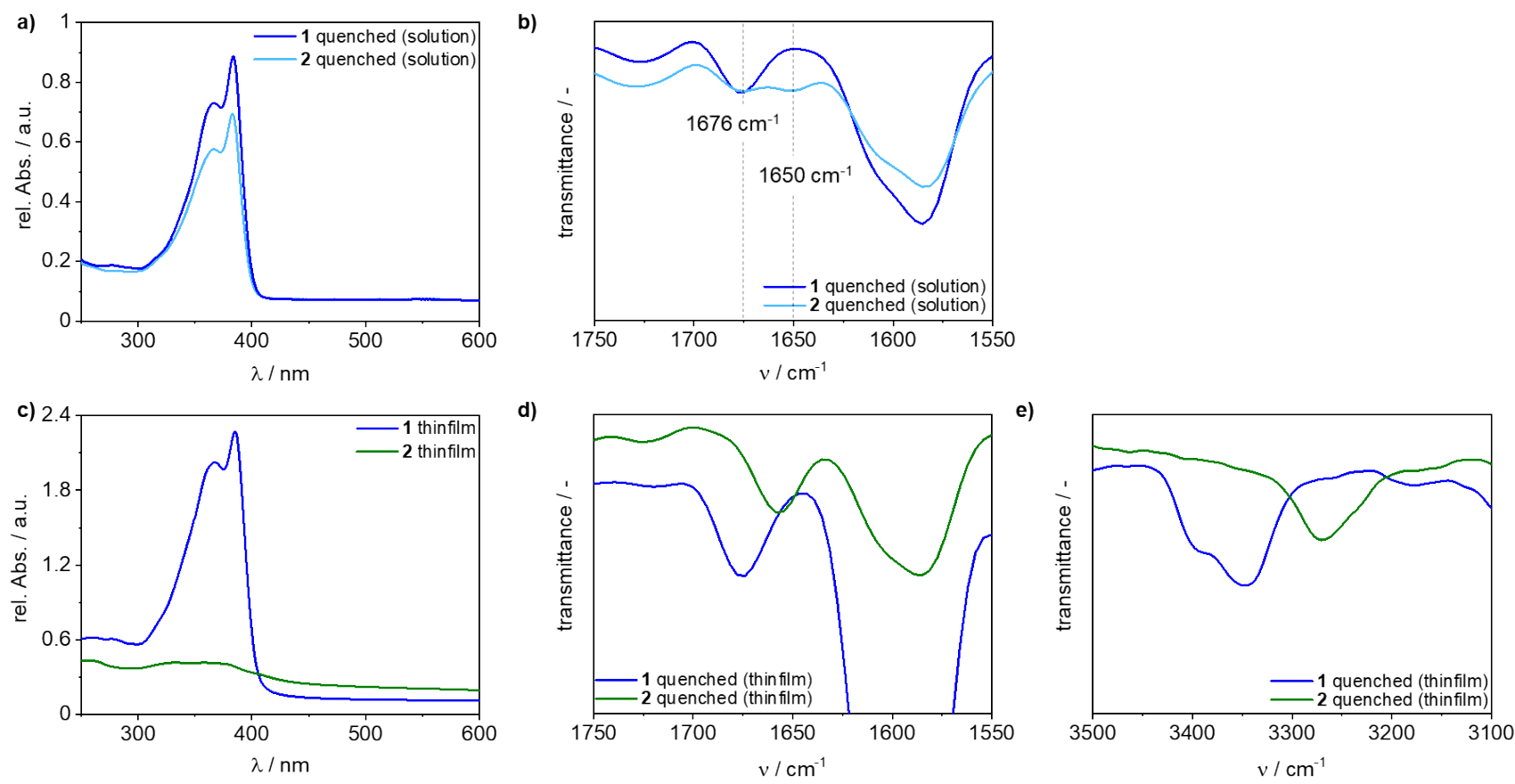

Figure S14. UV/Vis and FT-IR analysis of the packing mode/transient nature of slipped aggregates of $\mathbf{2}$ (cyan) in comparison to $\mathbf{1}$ (royal blue) in solution (top) and the solid state (bottom). The amide I carbonyl-stretching band of the slipped aggregate of $\mathbf{1}$ appears at $1675 \mathrm{~cm}^{-1}$ in solution as well as in the thin film. This unequivocally proves the presence of non-hydrogen bonded amide-carbonyl groups. In the case of 2 , two different bands are observed in solution due to the ongoing formation of $\mathrm{H}$-type assemblies: $v_{\mathrm{C}=0}=1676 \mathrm{~cm}^{-1}$ and $1650 \mathrm{~cm}^{-1}$. The former originates from free carbonyl groups as they are observed in the slipped assemblies, whereas the latter can be attributed to classical amide-to-amide H-bonds due to the ongoing formation of the $\mathrm{H}$-type assemblies. When the solution is evaporated, a significantly higher amount of $\mathrm{H}$-type aggregates (green) is formed, being the dominant species in the thin film (see also corresponding UV/Vis spectra). In the N-H stretching region, the quality of the spectra recorded in solution was not satisfying due to a higher degree of baseline noise, therefore only the spectra in the solid state are shown here. The slipped aggregates of $\mathbf{1}$, (which are maintained in the thin film) exhibit an N-H stretching frequency of $v_{\mathrm{N}-\mathrm{H}}=3348 \mathrm{~cm}^{-1}$, confirming the $\mathrm{N}-\mathrm{H} \cdots \mathrm{Cl}$ motif. In the case of 2 , the transformation to H-type aggregates upon preparation of the thin film, results in a significantly lower wavenumber of $v_{\mathrm{N}-\mathrm{H}}=3271 \mathrm{~cm}^{-1}$. This value is indicative of strong hydrogen bonding, as it would be the case for amide-to-amide hydrogen bonds.
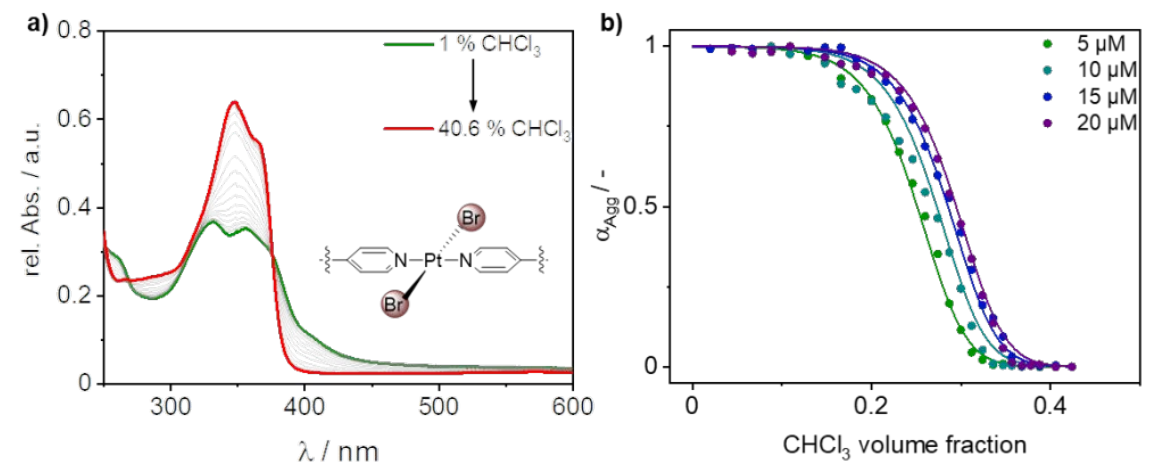

Figure S15. Denaturation studies of H-type aggregates of 2.a) UV/Vis spectra showing direct disassembly into the monomer (c $=5 \mu \mathrm{M}, \mathrm{T}=298 \mathrm{~K}$ ) $\mathrm{b}$ ) Aggregation curves derived from the apparent absorption at $400 \mathrm{~nm}$ at different concentrations and fit curves obtained with the cooperative denaturation model. 

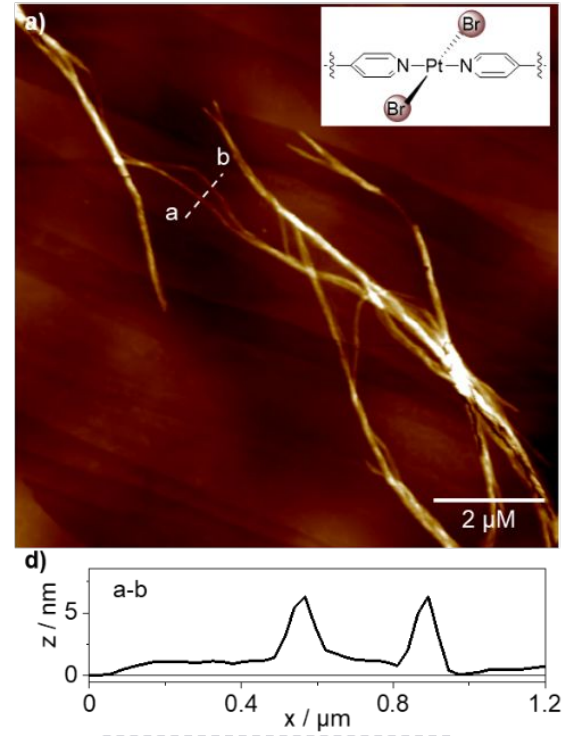

g)

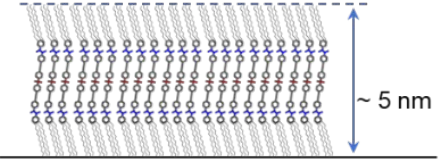

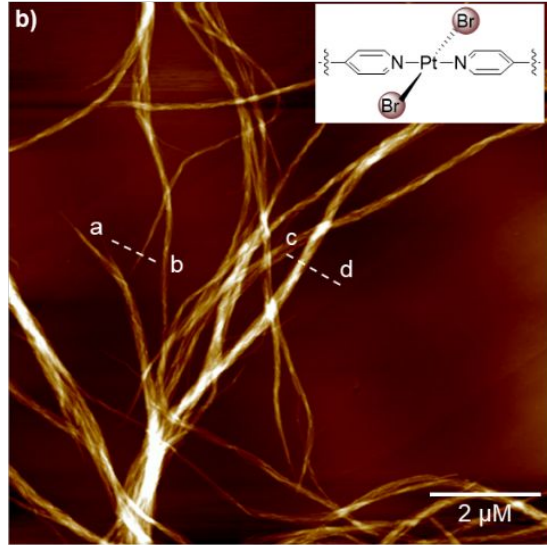
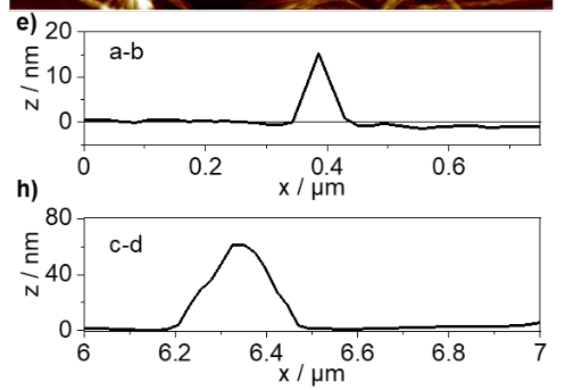
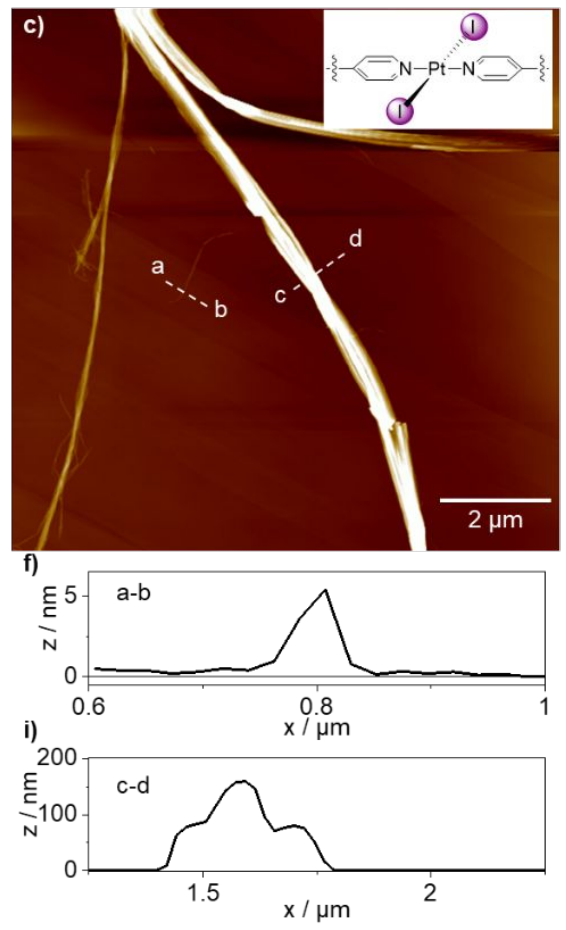

Figure S16. AFM analysis of H-type aggregates of $\mathbf{2}(\mathrm{a}, \mathrm{b})$ and $\mathbf{3}$ (c) with cross section analyses along a-b- and c-d axes. d) Height profile of two single $\mathrm{H}$-type aggregate strands of $\mathbf{2}$ with heights of $\sim 5 \mathrm{~nm}$. e) Height profile of a small stack of H-type aggregate strands of $\mathbf{2}$ with a height of $\sim 15 \mathrm{~nm}(\rightarrow$ three stacked strands). f) Height profile of one single H-type aggregate strand of $\mathbf{3}$ with a height of $\sim 5 \mathrm{~nm}$. g) Schematic representation of the orientation of the molecules within H-type assemblies on the HOPG surface, with the alkoxy chains pointing to the surface/the exterior. h) Height profile of a larger stack of $\mathrm{H}$-type aggregate strands of 2 with a height of $\sim 60 \mathrm{~nm}$. i) Height profile of a large stack of $\mathrm{H}$-type aggregate strands of 3 with a height of $\sim 160 \mathrm{~nm}$.

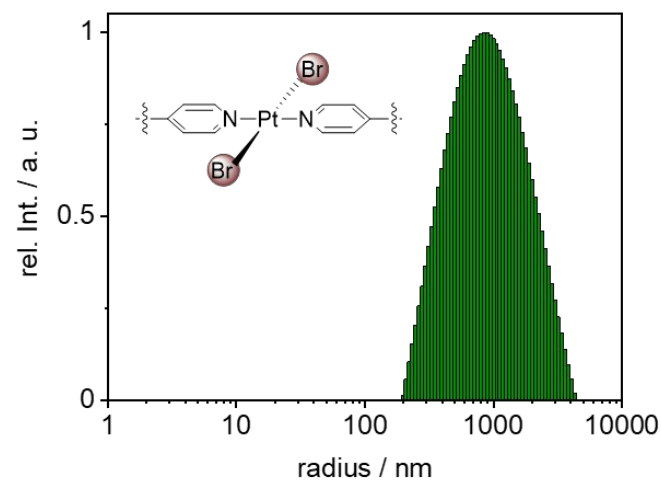

Figure S17. DLS size-distribution of solutions of H-type aggregates of $2(\mathrm{c}=10 \mu \mathrm{M}, T=293 \mathrm{~K})$.
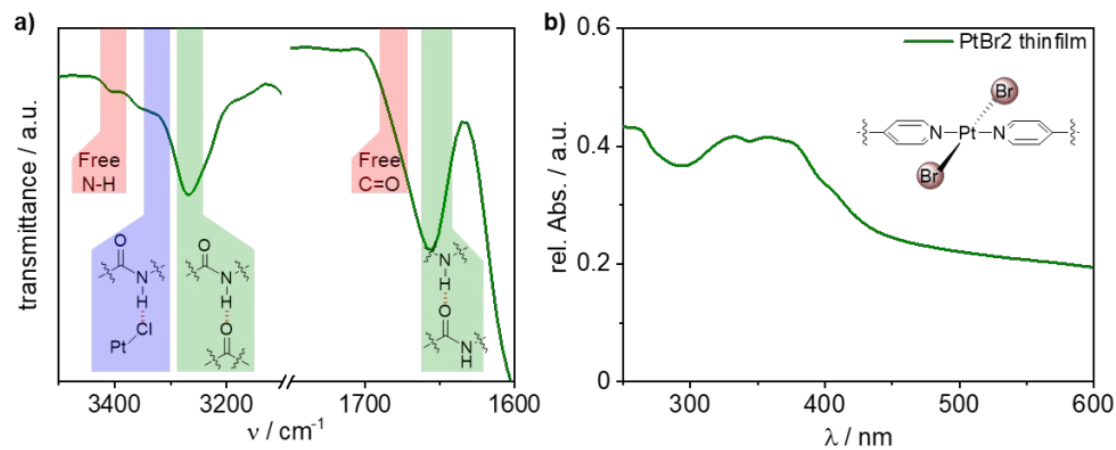

Figure S18. Thin-film FT-IR spectrum (a) of H-type aggregates of 2 in the N-H- and C=O stretching regions and corresponding UV/Vis spectrum (b). 

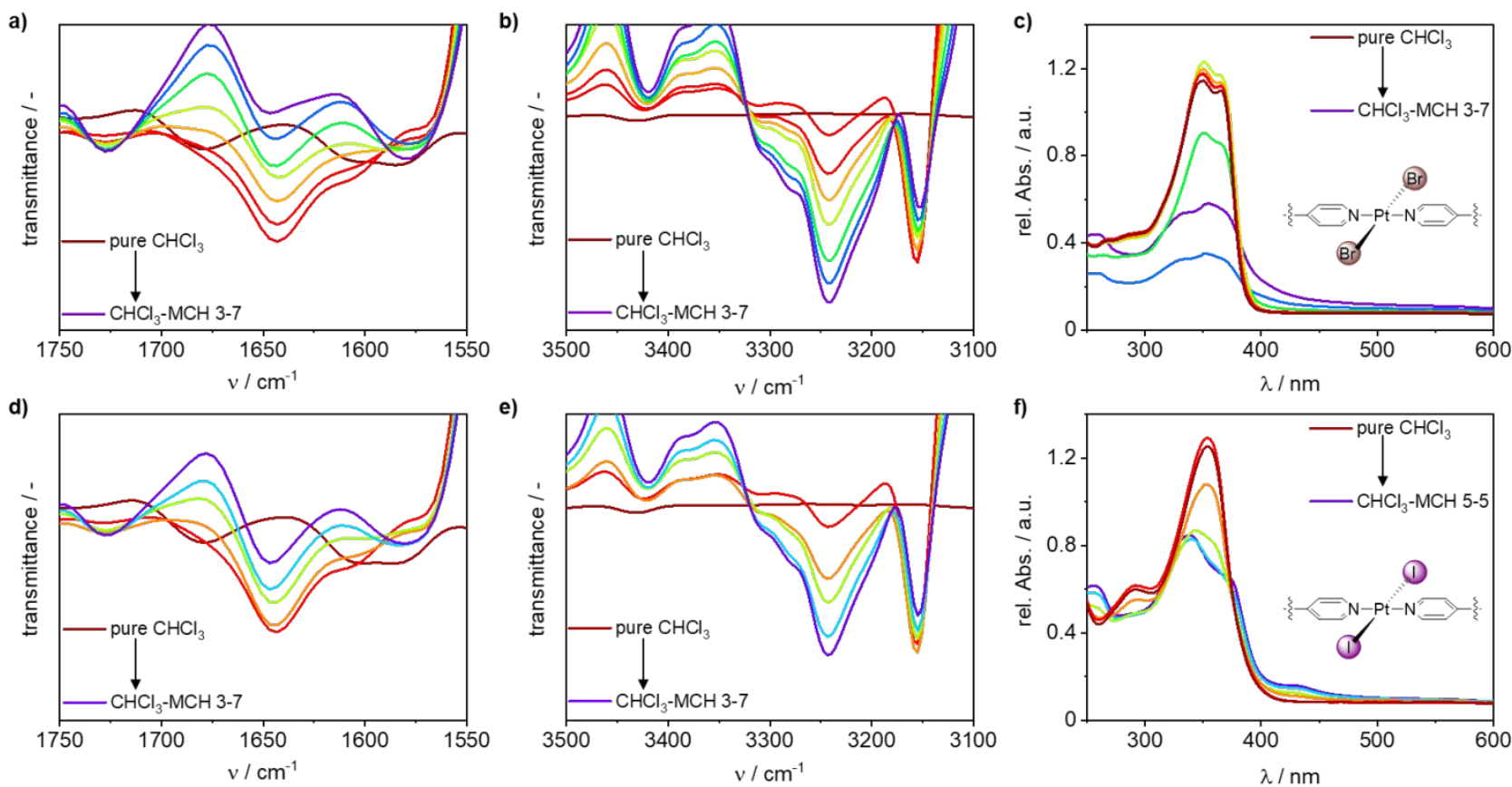

Figure S19. FT-IR and UV/Vis spectra following the polymerization of $\mathbf{1}$ (a-c) and $\mathbf{2}$ (d-f) upon increasing the $\mathrm{MCH} / \mathrm{CHCl}_{3}$ ratio $(\mathrm{c}=1 \mathrm{mM}$ ). The amide I stretching frequency immediately shifts from $1678 \mathrm{~cm}^{-1}$ to $1643 \mathrm{~cm}^{-1}$ for both 2 and 3 upon increasing the MCH content by $10 \mathrm{vol} \%$. Thereafter, the wavenumber does not change and only the intensities are affected by further decreasing the apolar fraction of the solvating medium. A similar trend is observed for the $\mathrm{N}-\mathrm{H}$ stretching frequency which is shifted from $3430 \mathrm{~cm}^{-1}$ to $3244 \mathrm{~cm}^{-1}$. The observation of similar changes in wavenumbers in the solid state support the correct assignment of a parallel-packing featuring amide-to-amide H-bonds.
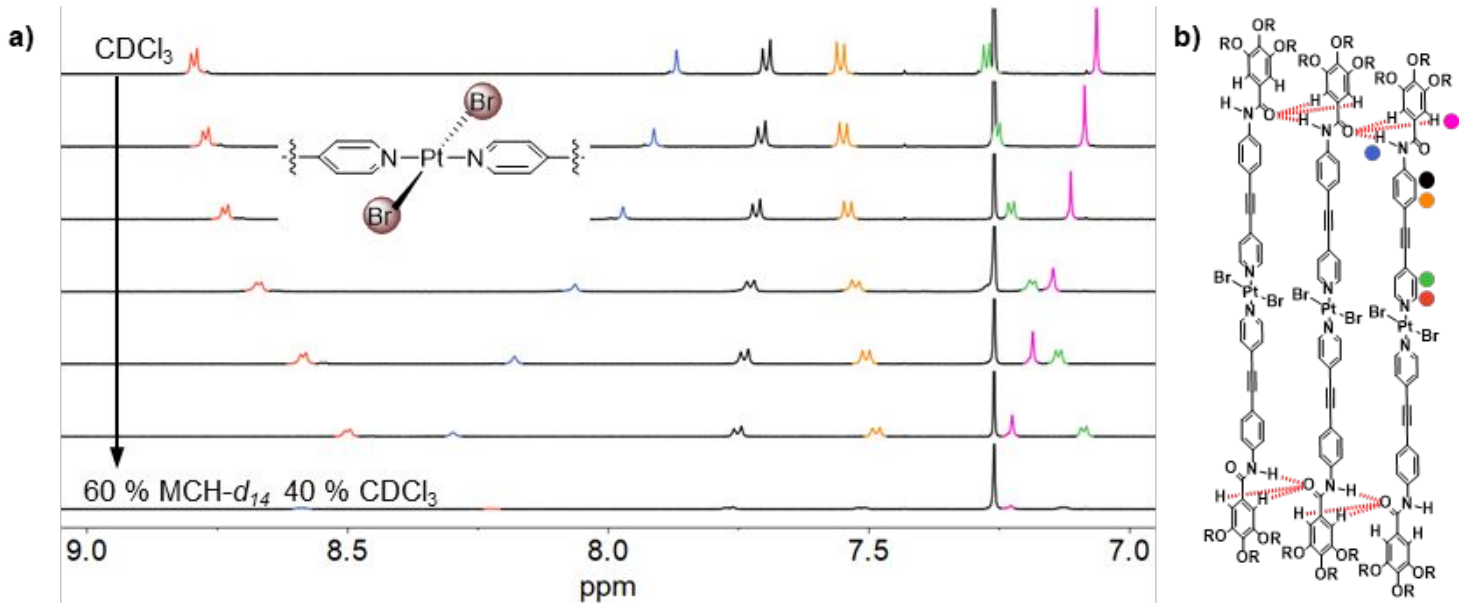

Figure S20. a) Changes of the ${ }^{1} \mathrm{H}$ NMR aromatic resonances of 2 upon formation of H-type aggregates by variation of the solvent composition (c = $1 \mathrm{mM}, T=298 \mathrm{~K}$ ). h) Parallel molecular structure illustrating the proton assignment and intermolecular interactions.

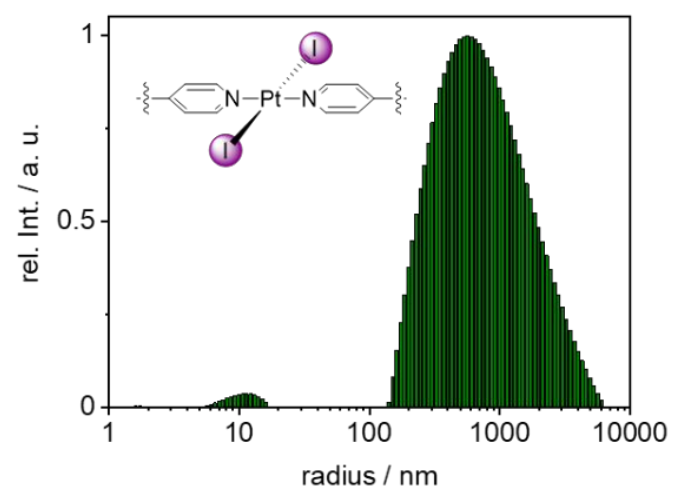

Figure S21. DLS size distribution of solutions of H-type aggregates of $3(c=10 \mu \mathrm{M}, T=293 \mathrm{~K})$. 

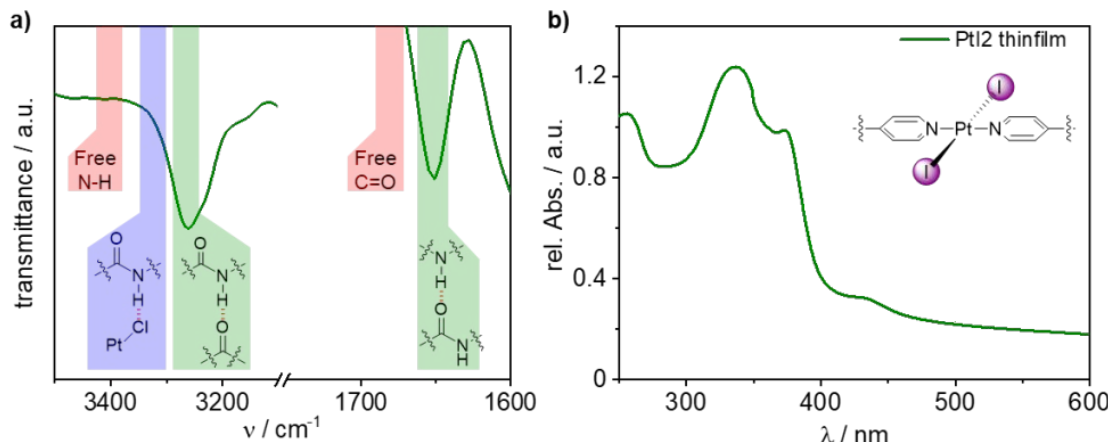

Figure S22. Thin-film FT-IR spectrum (a) of H-type aggregates of $\mathbf{3}$ in the N-H- and C=O stretching regions and corresponding UV/Vis spectrum (b).
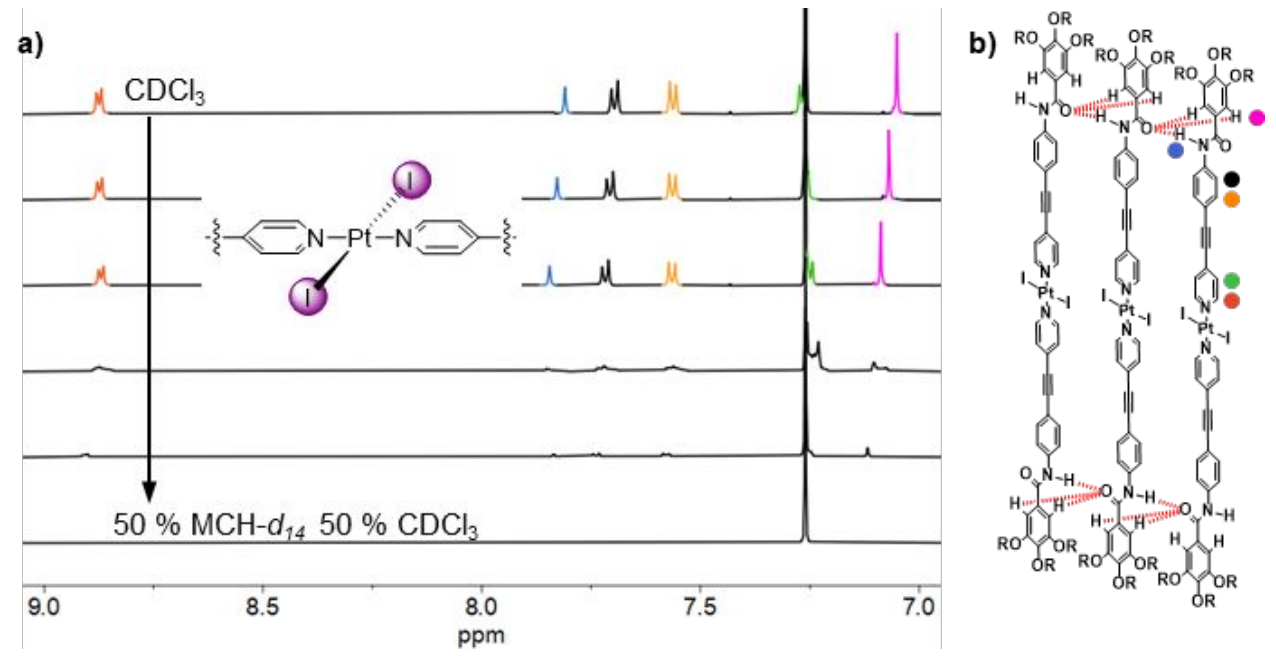

Figure S23. ${ }^{1} \mathrm{H}-\mathrm{NMR}$ resonances of $\mathbf{3}$ in the aromatic region following the polymerization of $\mathbf{3}$ upon increasing the $\mathrm{MCH}-\mathrm{d}_{14} / \mathrm{CDCl} \mathrm{l}_{3}$ ratio $(\mathrm{c}=1 \mathrm{mM}$, $\mathrm{T}=298 \mathrm{~K}$ ). Due to the fact that precipitation of the supramolecular polymers occurs rapidly after the addition of the apolar solvent at such high concentrations, the resonances of the residual complex in solution are still well resolved with only minor changes. At higher MCH- $d_{14}$ fractions, the majority of the complex is precipitated in polymerized form, resulting in very weak to no signals. However, throughout the first three spectra from the top, the amide- and gallic protons are deshielding due to intermolecular H-bonding, as identically observed for the H-type aggregate of 2 . In contrast to 2 , the resonances of the $\alpha$-pyridine protons are unaffected in 3 . Due to the increased steric hindrance at the Pt-center and hence a larger distance between two neighboring molecules at the center of the polymer, the effect of the ring-current of neighboring molecules is decreased around the pyridine moiety.

a)
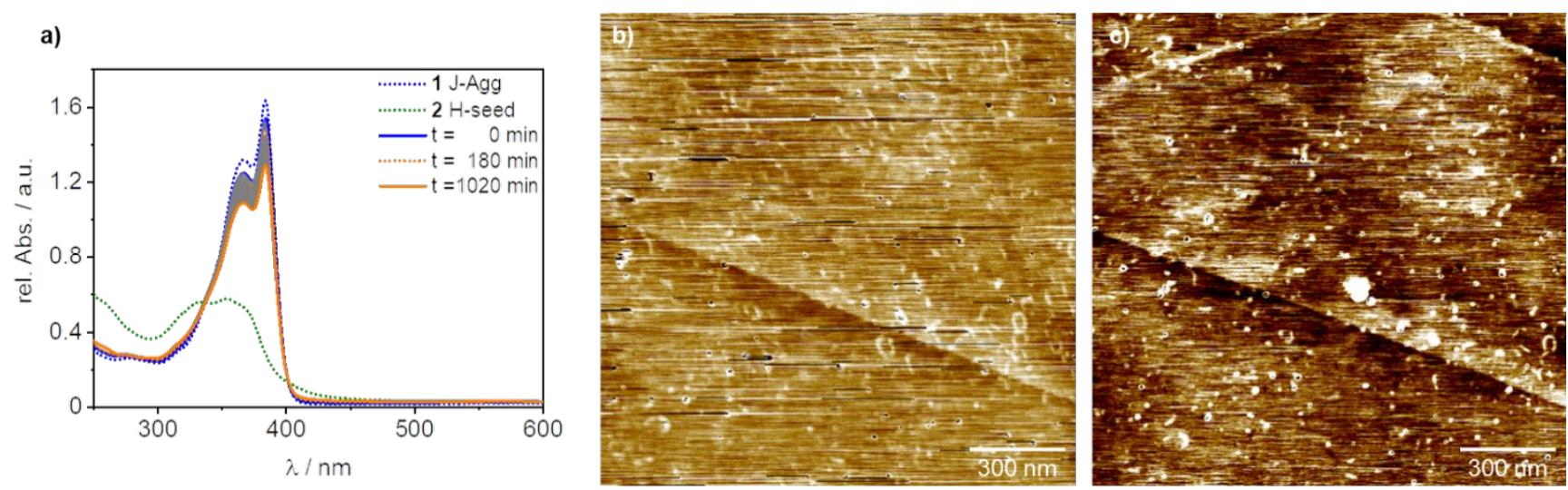

Figure S24. Hetero-seeding experiments with slipped aggregates of $\mathbf{1}$ and H-type seeds of 2. a) UV/Vis spectra of Slipped aggregates of $\mathbf{1}$ (blue, dotted), H-type seeds of 2 (green dotted) and time-dependent evolution of a 10-1 mixture of both (c $=10 \mu \mathrm{M}, T=298 \mathrm{~K}, \mathrm{MCH}-\mathrm{CHCl} 39-1)$. The mixture of H-type seeds and slipped aggregates was sonicated at $\mathrm{t}=180 \mathrm{~min}$ (orange, dotted), in order to open closed-ring slipped aggregates. b,c) AFMimages of a solution of sonicated slipped aggregates of $\mathbf{1}$ (drop casted onto HOPG), showing opened ring-structures and spherical polymer-fragments. 


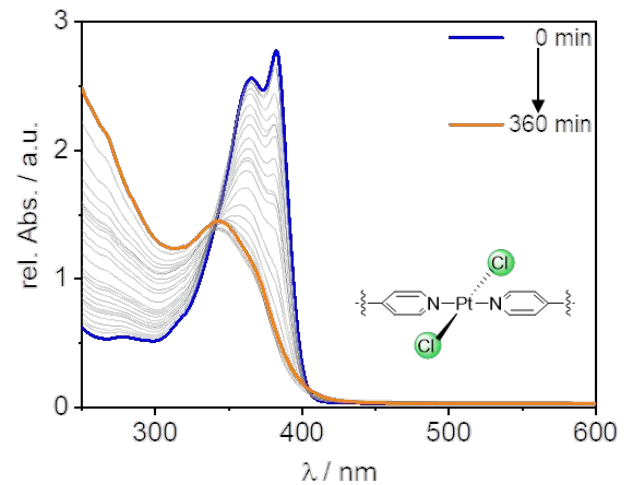

Figure S25. Time-dependent development of the absorption spectra of a solution of slipped aggregates of $\mathbf{1}$ upon prolonged sonication.
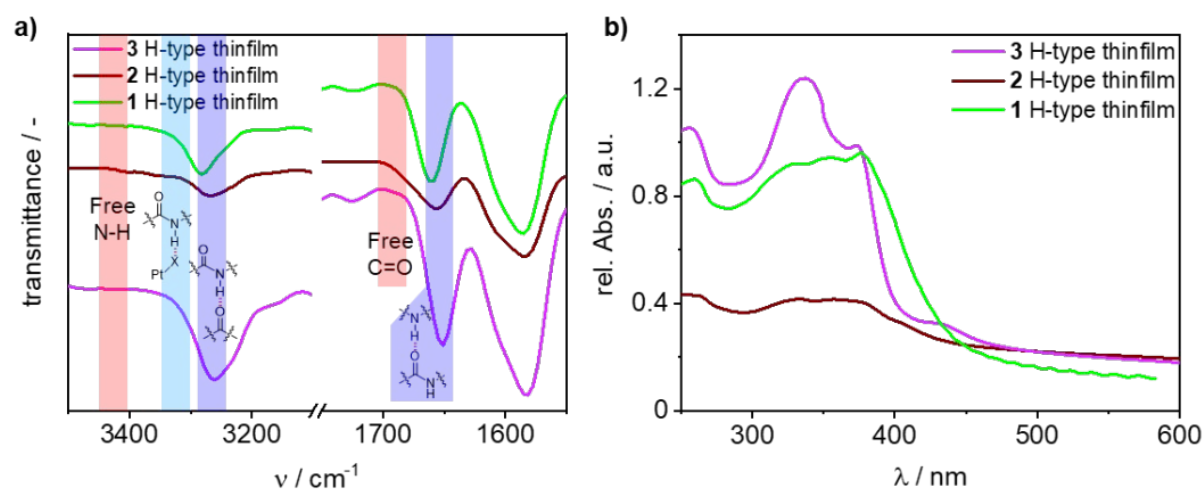

Figure S26. a) Complied FT-IR spectra of H-type aggregates of $\mathbf{1}-\mathbf{3}$ in the $\mathrm{N}-\mathrm{H}$ and amide I stretching region. The colored areas mark the spectral region in which the bands of non-hydrogen bonded (red), $\mathrm{N}-\mathrm{H} \cdots \mathrm{Cl}$ hydrogen bonded (cyan) and amide-to-amide H-bonded species (blue) appear. b) corresponding UV/Vis spectra illustrating the presence of H-type polymers in the thin films.

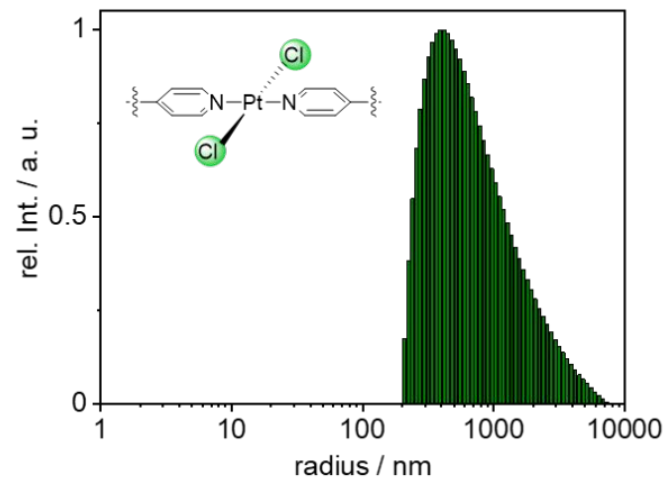

Figure S27. DLS size-distributions obtained of solutions of H-type aggregates of $\mathbf{1}(\mathrm{MCH}, \mathrm{c}=10 \mu \mathrm{M}, \mathrm{T}=293 \mathrm{~K})$.
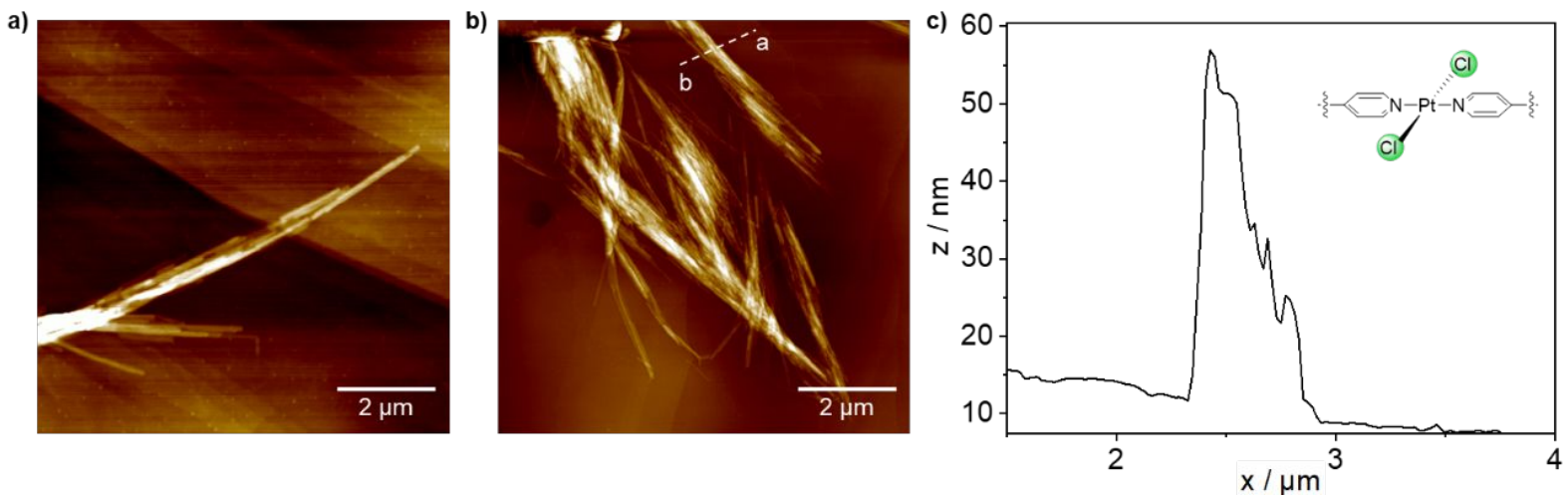

Figure S28. a,b) AFM images of H-type aggregates of 1, drop casted onto HOPG (10 $\mu \mathrm{M}, T=298 \mathrm{~K})$. c) Height profile analysis along the a-b axis in (b). 

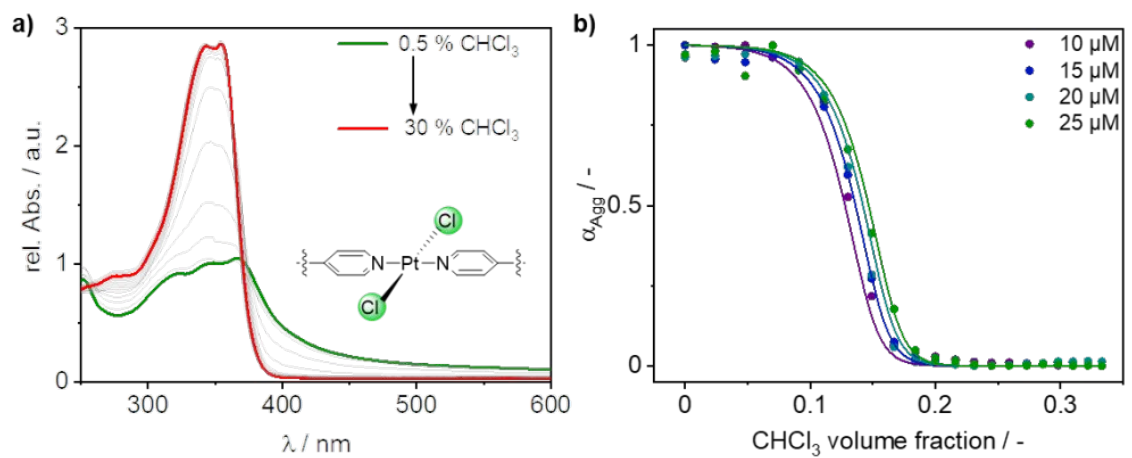

Figure S29. Denaturation studies of H-type aggregates of 1. a) UV/Vis spectra showing direct disassembly into the monomer (c $=20 \mu \mathrm{M}, T=298 \mathrm{~K})$ b) Aggregation curves derived from the apparent absorption at $348 \mathrm{~nm}$ at different concentrations and fit curves obtained with the cooperative denaturation model.

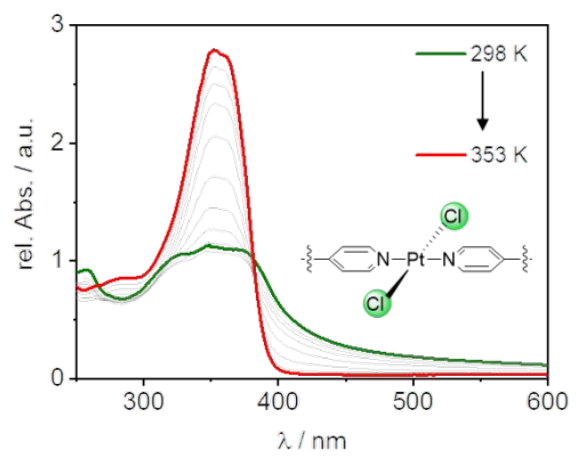

Figure S30. UV/Vis-heating studies of H-type aggregates of $\mathbf{1}$ showing direct disassembly into the monomer $(\mathrm{c}=25 \mu \mathrm{M})$. 


\section{Theoretical Calculations}

The geometries of monomers, dimers and tetramers in a slipped and parallel packing mode of all three complexes were optimized using the semiempirical PM7 method ${ }^{5}$ as implemented in the MOPAC package. ${ }^{6}$ Dispersion interactions were considered and all calculations were carried out in vacuum. The reliability of the optimized structures was checked via frequency calculations, through the absence of negative (imaginary) frequencies.

Density Functional Theory (DFT) calculations were performed using the QChem package ${ }^{7}$ (version 5.3.1) to further refine the geometry of all pre-optimized dimers via a fully unconstrained geometry optimization. The long side chains were substituted by methoxy groups ( $\rightarrow$ compounds 1-OMe, 2-OMe, 3-OMe) to make the DFT calculations feasible. The DFT functional B3LYP ${ }^{8,9}$ was used, together with the basis set 6-31G for Pt, Cl, Br, I and for the light atoms the basis set 3-21G was adopted. For the four heavier atoms the LANL2DZ effective core potential was also used. The Grimme dispersion correction was employed in all calculations.

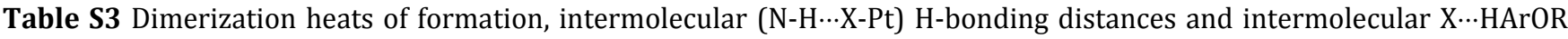
bond distances obtained from semiempirical PM7 calculations in vacuum. Data for the slipped aggregate (stabilized by NH...X-Pt H-bonds).

\begin{tabular}{|c|c|c|c|}
\hline X-aggregate (slipped) & $\Delta \mathrm{H}_{\mathrm{f}} / \mathrm{kJ} \mathrm{mol}^{-1}$ & $\mathrm{~N}-\mathrm{H} \cdots \mathrm{X} / \AA$ & 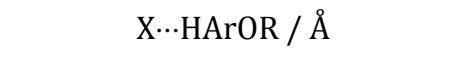 \\
\hline Cl-monomer & -1984.60 & - & - \\
\hline Cl-dimer & -4429.93 & $2.63 / 2.68(\varnothing=2.66)$ & $3.09 / 3.02(\varnothing=3.06)$ \\
\hline Cl-tetramer & -9465.13 & $\begin{array}{c}2.55 / 2.60 / 2.99 / 2.62 / 2.62 / 3.00 \\
(\varnothing=2.73)\end{array}$ & $\begin{array}{c}2.79 / 4.00 / 3.67 / 3.05 / 3.03 / 2.77 \\
(\varnothing=3.22)\end{array}$ \\
\hline Br-monomer & -1943.62 & - & - \\
\hline Br-dimer & -4297.37 & $2.84 / 2.87(\varnothing=2.86)$ & $3.25 / 3.33(\varnothing=3.29)$ \\
\hline Br-tetramer & -9009.46 & $\begin{array}{c}2.67 / 2.81 / 2.84 / 2.99 / 2.82 / 3.24 \\
(\varnothing=2.90)\end{array}$ & $\begin{array}{c}3.19 / 3.33 / 3.78 / 3.64 / 2.89 / 3.41 \\
(\varnothing=3.37)\end{array}$ \\
\hline I-monomer & -1873.56 & - & - \\
\hline I-dimer & -4154.57 & $3.02 / 3.28(\varnothing=3.15)$ & $3.64 / 3.43(\varnothing=3.54)$ \\
\hline I-tetramer & -8992.3 & $\begin{array}{c}3.57 / 3.08 / 3.18 / 3.16 / 3.54 / 3.02 \\
(\varnothing=3.26)\end{array}$ & $\begin{array}{c}3.13 / 3.38 / 3.64 / 4.48 / 3.28 / 3.56 \\
(\varnothing=3.58)\end{array}$ \\
\hline
\end{tabular}

$\Delta \mathrm{H}\left(\right.$ dimerization, $\left.\mathrm{kJ} \mathrm{mol}^{-1}\right)=-460.73(\mathrm{Cl}),-428.13(\mathrm{Br}),-407.45(\mathrm{I})$

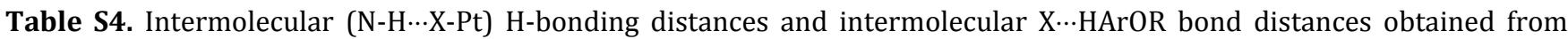
DFTcalculations in vacuum. Data for the slipped aggregate (stabilized by N-H $\cdots X$-Pt H-bonds).

\begin{tabular}{|l|c|c|}
\hline X-aggregate (slipped) & $\mathrm{N}-\mathrm{H} \cdots \mathrm{X} / \AA$ & $\mathrm{X} \cdots \mathrm{HArOR} / \AA$ \\
\hline Cl-dimer & $2.48 / 3.60(\varnothing=3.04)$ & $2.85 / 3.66(\varnothing=3.26)$ \\
\hline Br-dimer & $2.65 / 3.52(\varnothing=3.09)$ & $3.12 / 4.04(\varnothing=3.58)$ \\
\hline I-dimer & $2.83 / 3.75(\varnothing=3.29)$ & $3.26 / 4.09(\varnothing=3.68)$ \\
\hline
\end{tabular}


a)

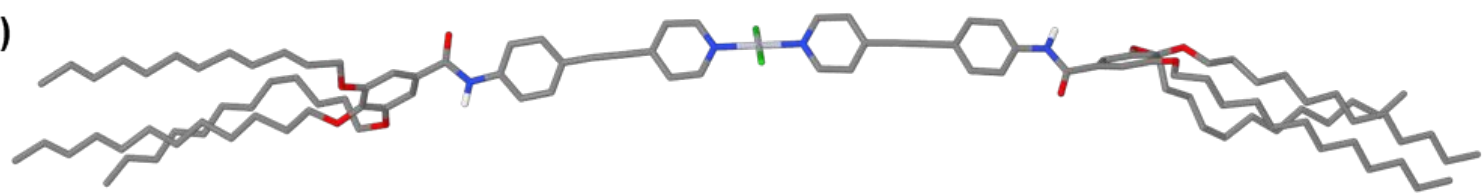

b)

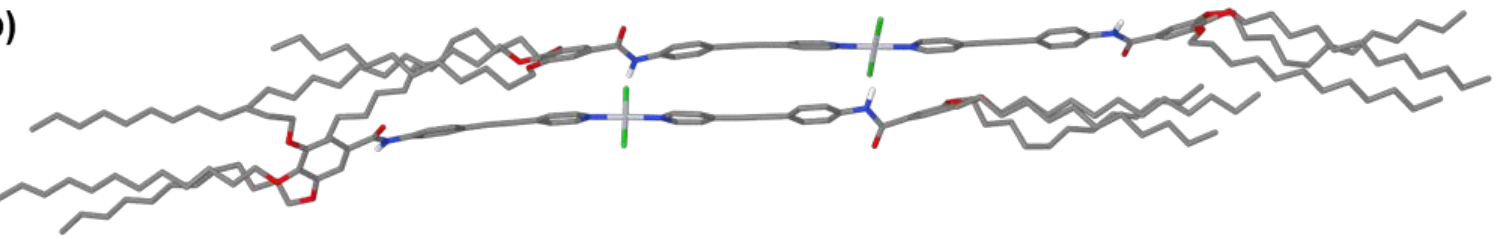

c)

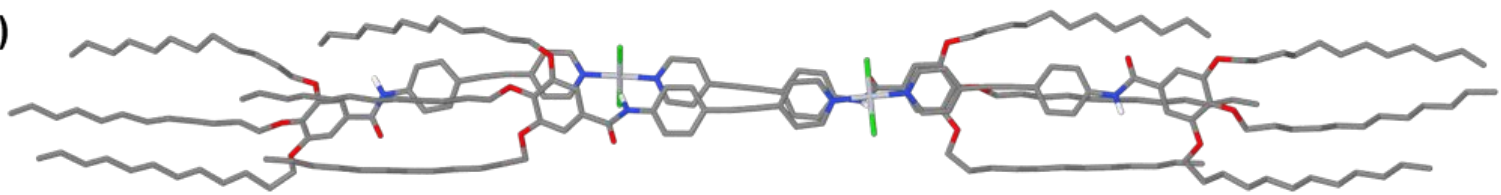

d)

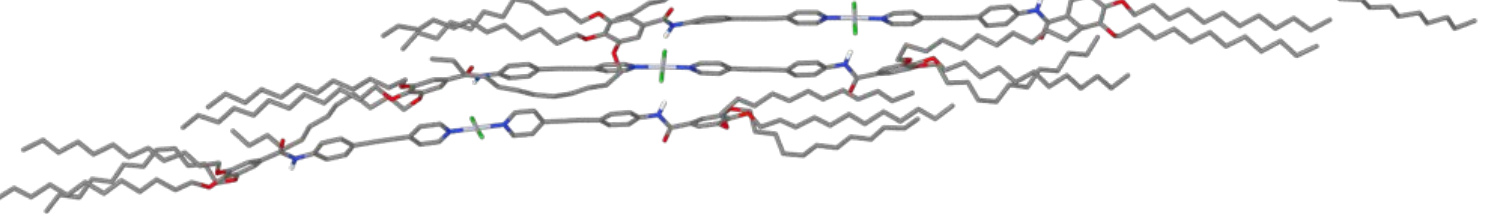

e)

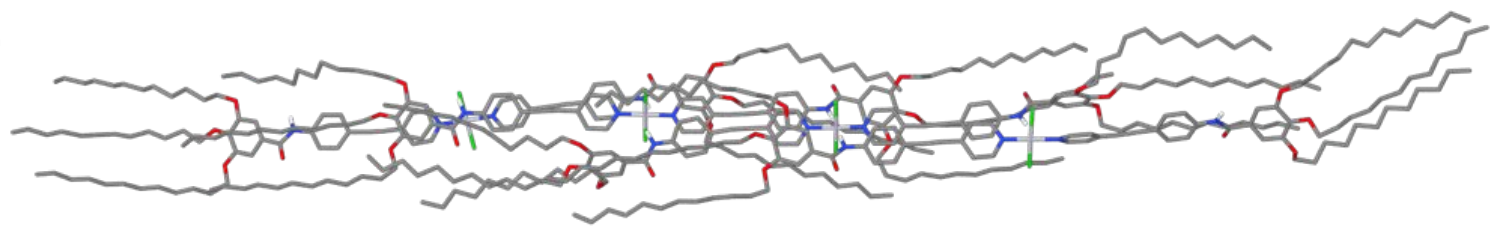

Figure S31. Geometry optimized structures (semiempirical PM7 method) of a slipped aggregate of 1. a) monomer, b) dimer side view, c) dimer topview, d) tetramer side view, e) tetramer top view.

a)
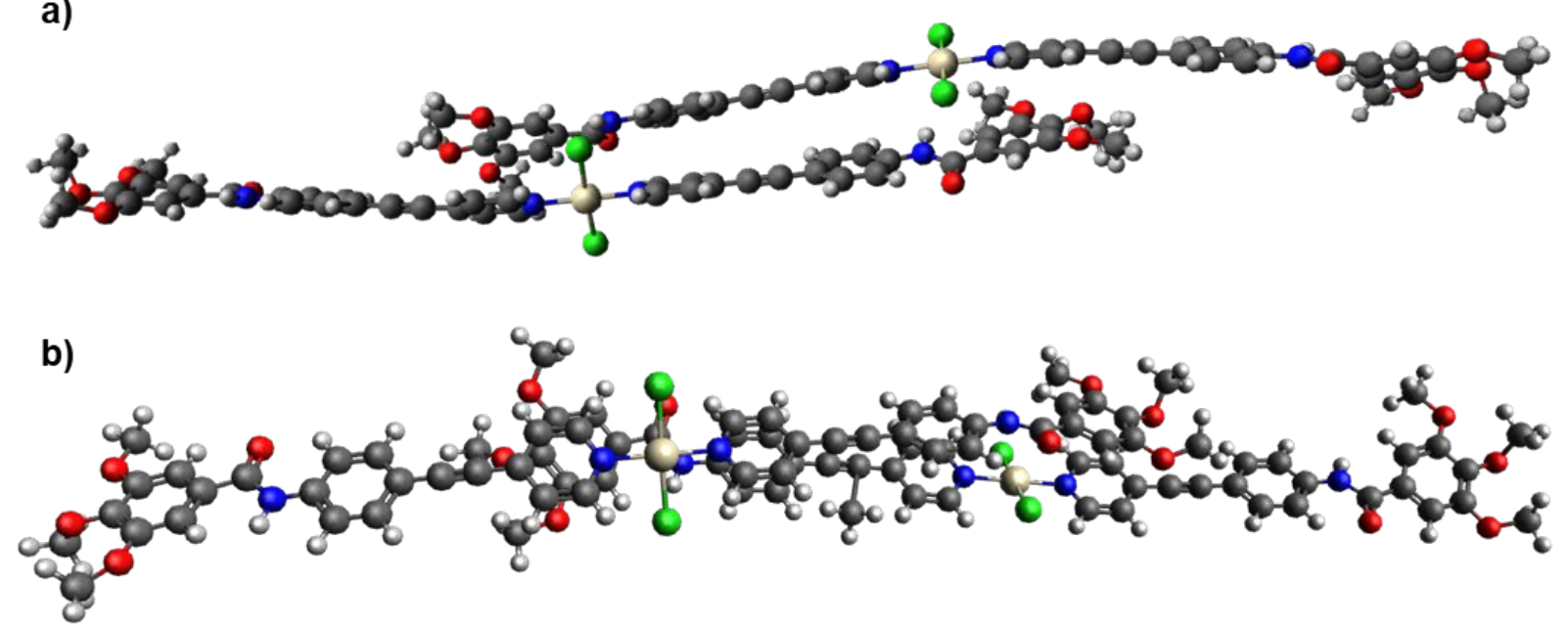

Figure S32. Geometry optimized structures (DFT) of a slipped aggregate of 1-OMe. a) side view, b) top-view. 
a)

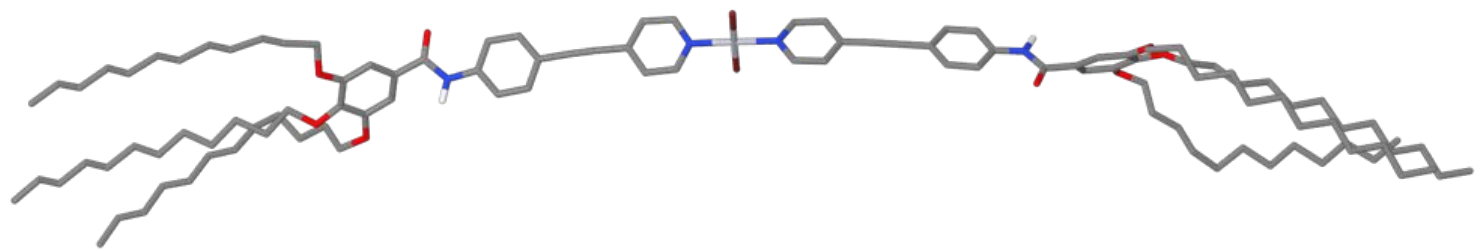

b)

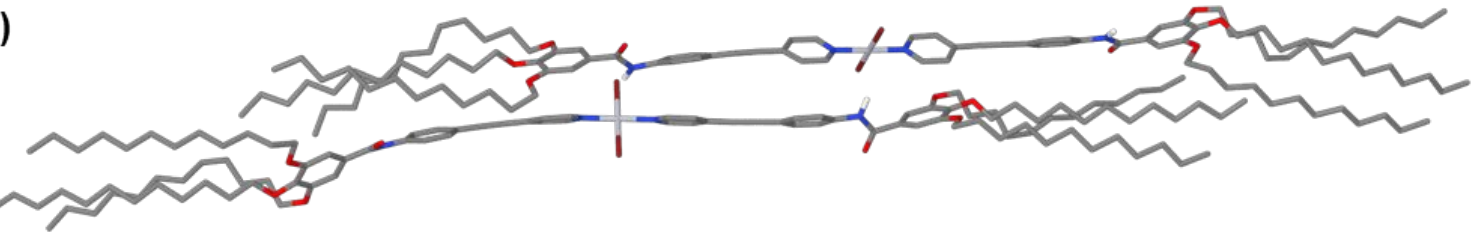

c)

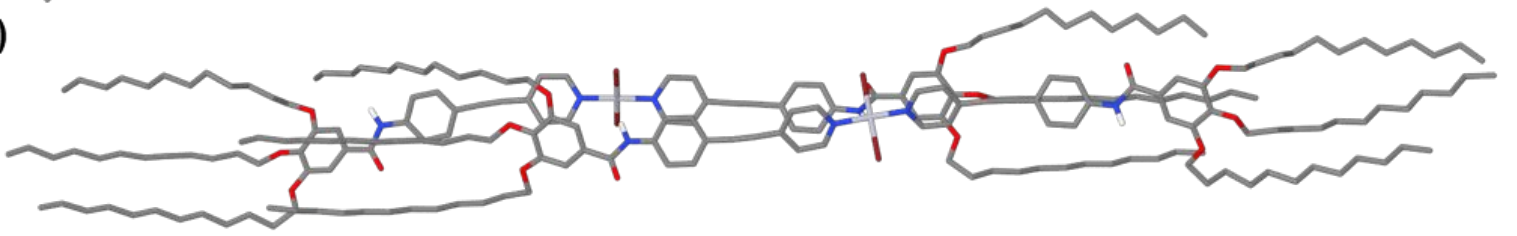

d)
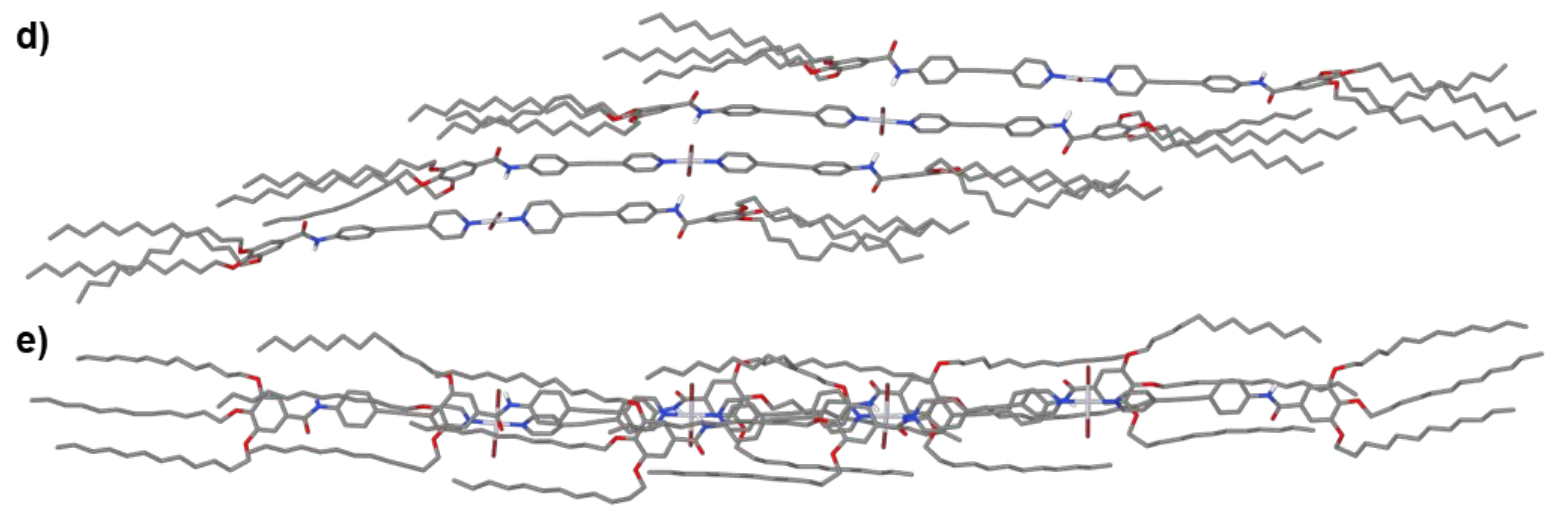

Figure S33. Geometry optimized structures (semiempirical PM7 method) of a slipped aggregate of 2. a) monomer, b) dimer side view, c) dimer topview, d) tetramer side view, e) tetramer top view.
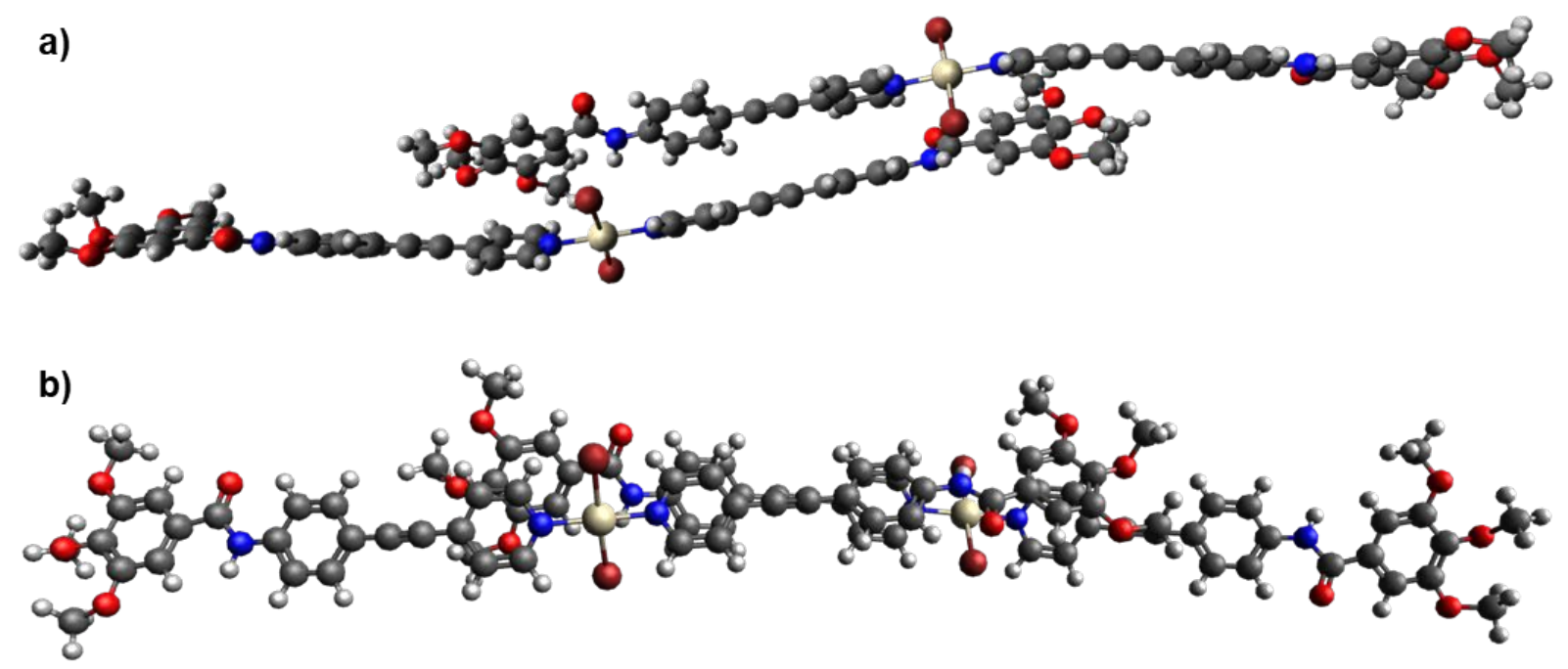

Figure S34. Geometry optimized structures (DFT) of a slipped aggregate of 2-0Me. a) side view, b) top-view. 
a)

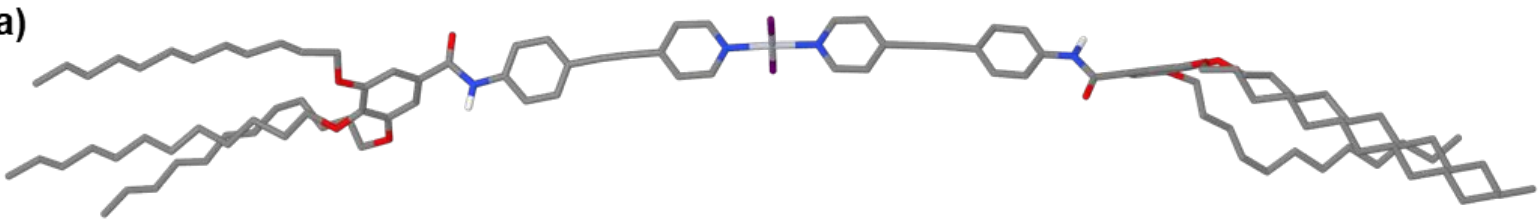

b)

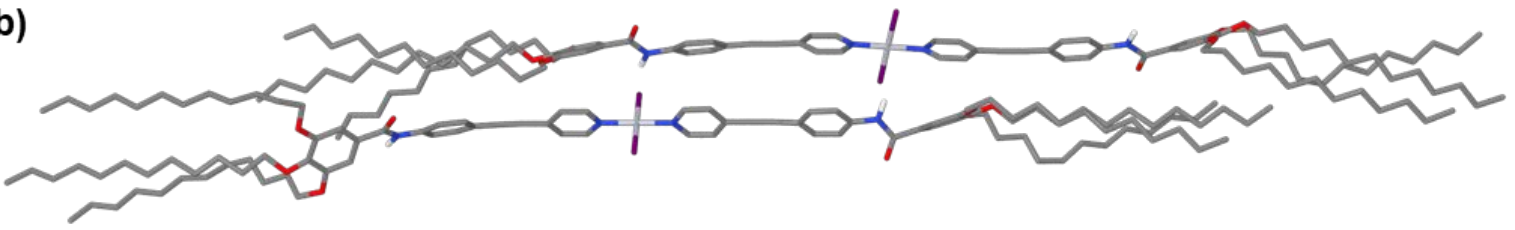

c)

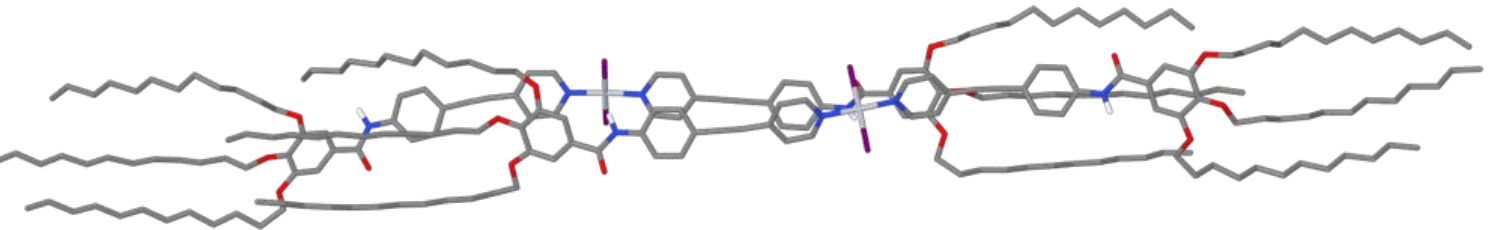

d)

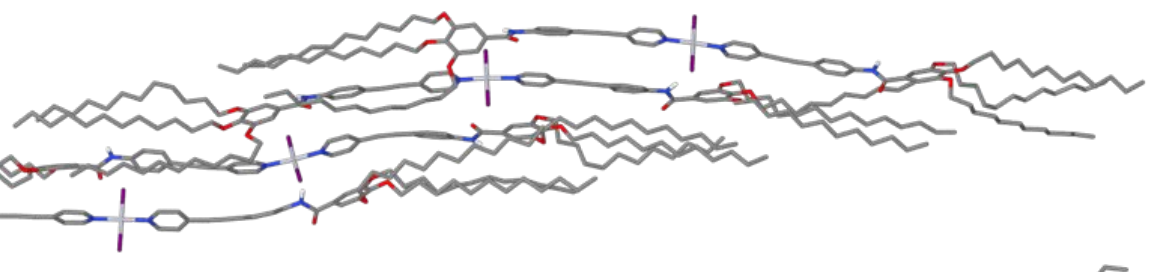

e)

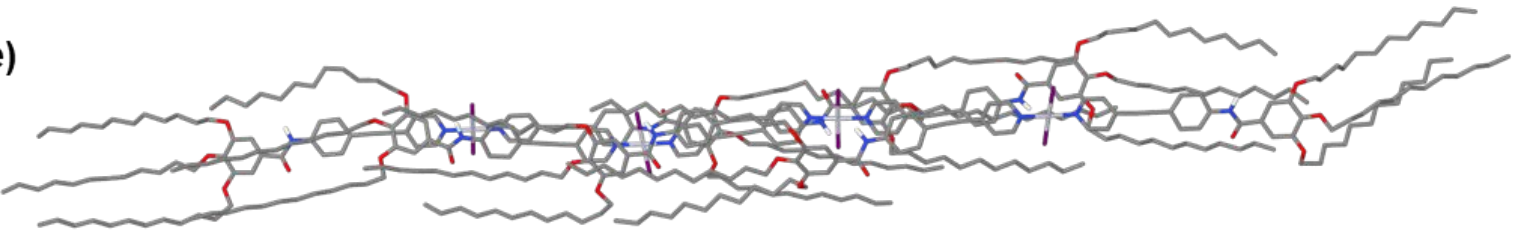

Figure S35. Geometry optimized structures (semiempirical PM7 method) of a hypothetic slipped aggregate of 3. a) monomer, b) dimer side view, c) dimer top-view, d) tetramer side view, e) tetramer top view. Note the strong, unfavorable twist along the long axis of the molecule, which does not occur for $\mathbf{1}$ or $\mathbf{2}$.
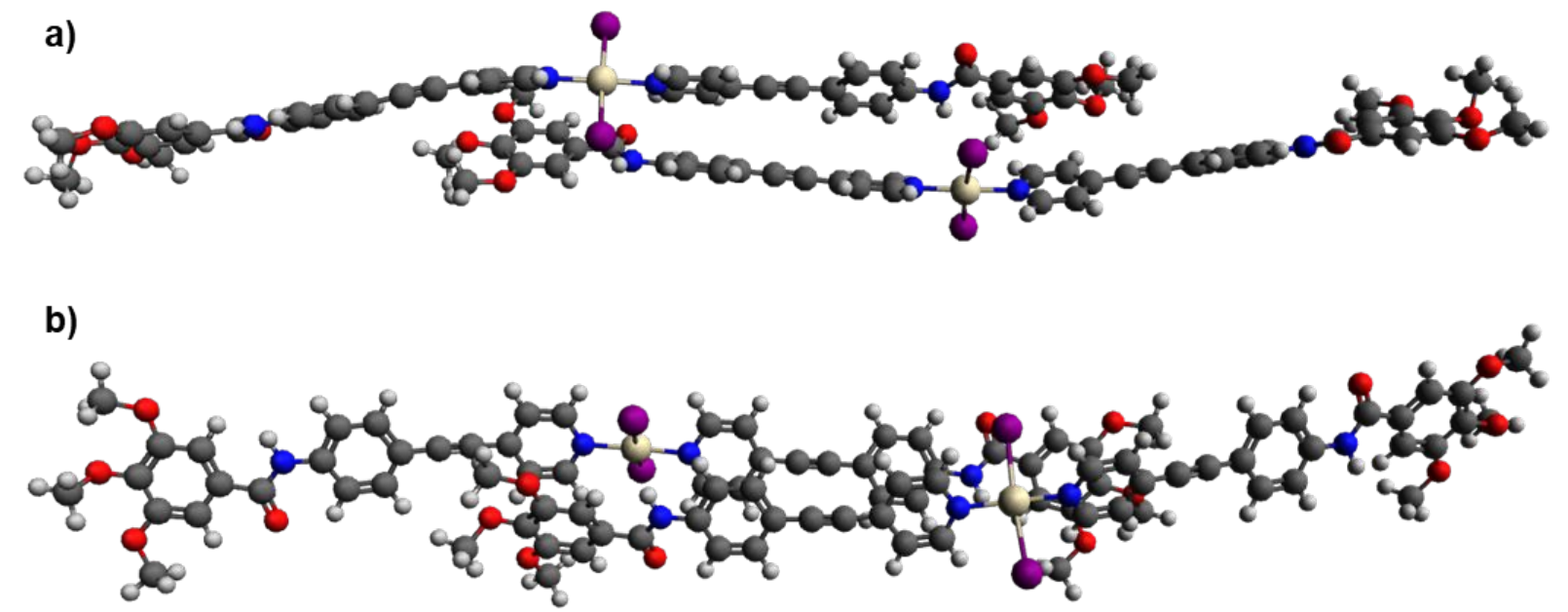

Figure S36. Geometry optimized structures (DFT) of a slipped aggregate of 3-OMe. a) side view, b) top-view. 
Table S5. Dimerization heats of formation, intermolecular ( $\mathrm{N}-\mathrm{H} \cdots \mathrm{O}=\mathrm{C}) \mathrm{H}$-bonding distances and intermolecular X-Pt and Pt-Pt distances obtained from semiempirical PM7 calculations in vacuum. Data for the parallel aggregate (stabilized by amide-toamide H-bonds).

\begin{tabular}{|c|c|c|c|c|}
\hline $\begin{array}{l}\text { X-aggregate } \\
\text { (slipped) }\end{array}$ & $\Delta \mathrm{H}_{\mathrm{f}} / \mathrm{kJ} \mathrm{mol}^{-1}$ & $\mathrm{~N}-\mathrm{H} \cdots \mathrm{O}=\mathrm{C} / \AA$ & $\mathrm{X} \cdots \mathrm{Pt} / \AA$ & Pt...Pt / $\AA$ \\
\hline Cl-monomer & -1984.60 & - & - & - \\
\hline Cl-dimer & -4571.98 & $1.99 / 2.06(\varnothing=2.03)$ & 4.08 & 4.4 \\
\hline Cl-tetramer & -9850.21 & $\begin{array}{c}2.00 / 1.91 / 1.97 / 2.11 / 1.85 / 1.96 \\
(\varnothing=1.97)\end{array}$ & 3.79 & $\begin{array}{c}4.09 / 4.04 / 4.11 \\
(\varnothing=4.08)\end{array}$ \\
\hline Br-monomer & -1943.62 & - & - & - \\
\hline Br-dimer & -4467.90 & $2.00 / 2.07(\varnothing=2.04)$ & 4.37 & 4.73 \\
\hline Br-tetramer & -9632.22 & $\begin{array}{c}1.98 / 1.87 / 1.96 / 1.96 / 1.85 / 2.12 \\
(\varnothing=1.96)\end{array}$ & 3.99 & $\begin{array}{c}4.32 / 4.20 / 4.37 \\
(\varnothing=4.30) \\
\end{array}$ \\
\hline I-monomer & -1873.56 & - & - & - \\
\hline I-dimer & -4339.44 & $1.97 / 2.10(\varnothing=2.04)$ & 4.73 & 5.15 \\
\hline I-tetramer & -9373.39 & $\begin{array}{c}1.99 / 1.88 / 1.94 / 1.96 / 1.85 / 2.08 \\
(\varnothing=1.95)\end{array}$ & 4.45 & $\begin{array}{c}4.74 / 4.26 / 4.80 \\
(\varnothing=4.60)\end{array}$ \\
\hline
\end{tabular}

$\Delta \mathrm{H}\left(\right.$ dimerization, $\left.\mathrm{kJ} \mathrm{mol}^{-1}\right)=-602.78(\mathrm{Cl}),-598.66(\mathrm{Br}),-592.32(\mathrm{I})$

$\pi$ - $\pi$ distances: $3.60 \AA$ ( $\mathrm{Cl}$ ), for $\mathrm{Br}$ and I the $\pi$-system is not very regular or planar to get a single $\pi$-distance

Table S6. Intermolecular $(\mathrm{N}-\mathrm{H} \cdots \mathrm{O}=\mathrm{C}) \mathrm{H}$-bonding distances and intermolecular X $\cdots \mathrm{Pt}$ and Pt $\cdots \mathrm{Pt}$ distances obtained from DFT calculations in vacuum. Data for the H-type aggregate (stabilized by amide-to-amide H-bonds).

\begin{tabular}{|l|c|c|c|}
\hline X-aggregate (H-type) & $\mathrm{N}-\mathrm{H} \cdots \mathrm{O}=\mathrm{C} / \AA$ & $\mathrm{X} \cdots \mathrm{Pt} / \AA$ & $\mathrm{Pt} \cdots \mathrm{Pt} / \AA$ \\
\hline Cl-dimer & $1.84 / 1.89(\varnothing=1.87)$ & $3.89 / 3.97(\varnothing=3.93)$ & 3.67 \\
\hline Br-dimer & $1.93 / 1.90(\varnothing=1.92)$ & $4.07 / 4.07(\varnothing=4.07)$ & 3.90 \\
\hline I-dimer & $1.80 / 1.91(\varnothing=1.86)$ & $4.23 / 4.23(\varnothing=4.23)$ & 4.19 \\
\hline
\end{tabular}


a)

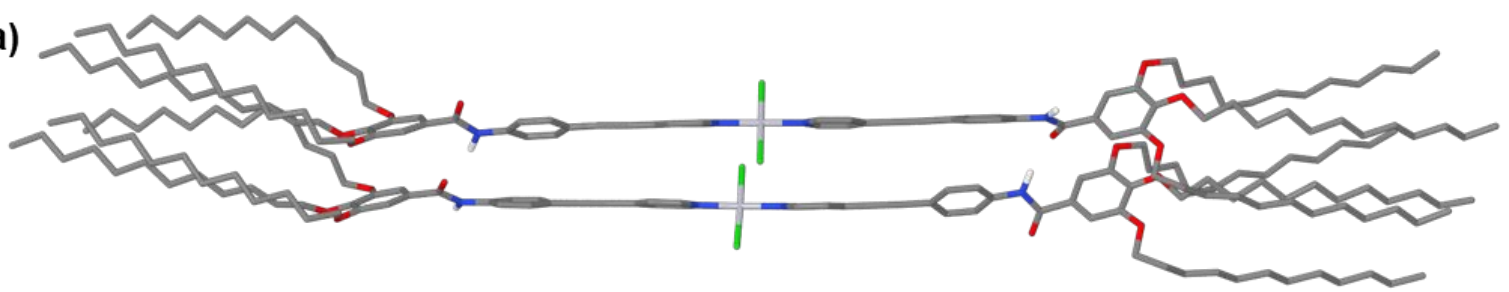

b)

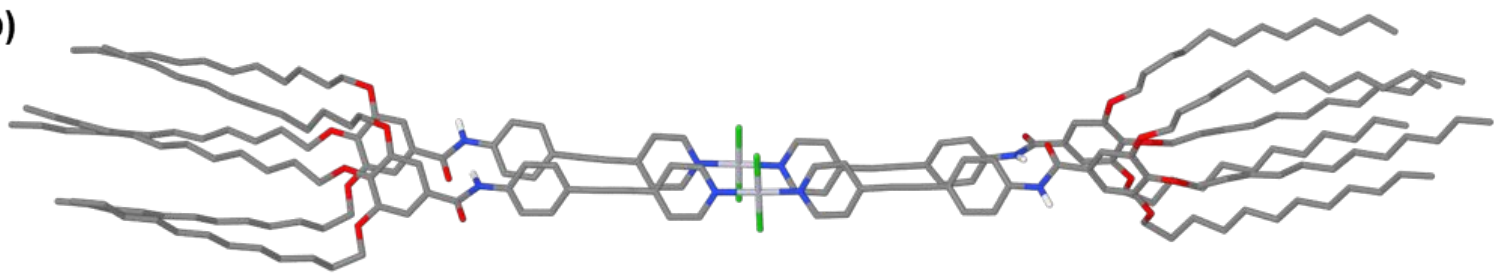

c)

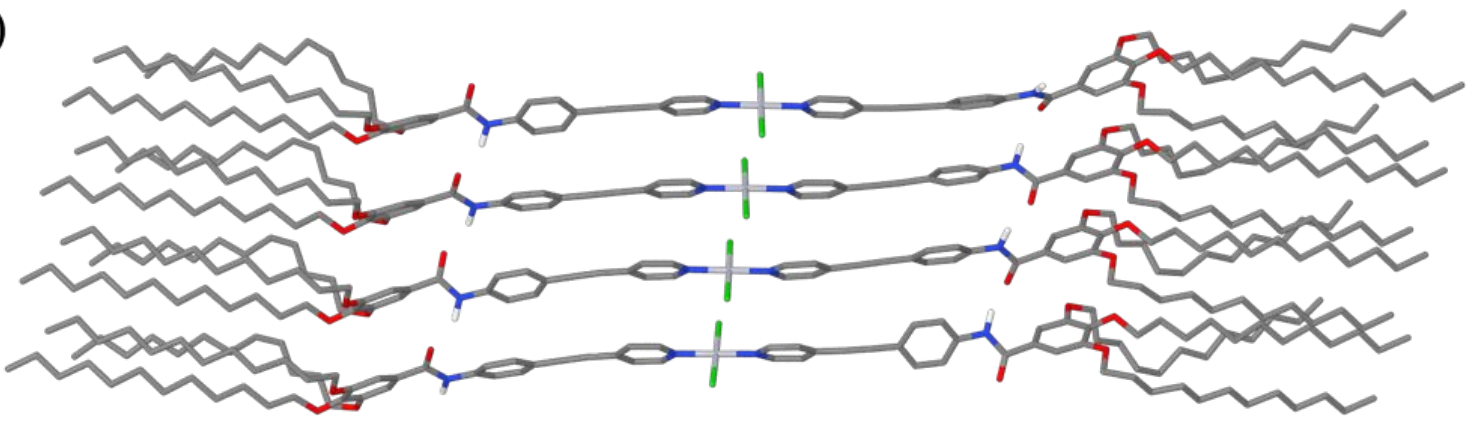

d)

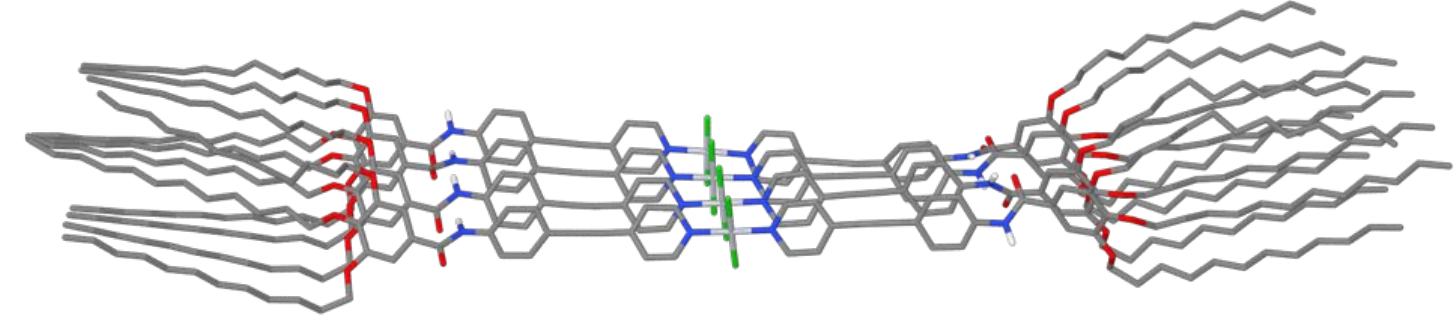

Figure S37. Geometry optimized structures (semiempirical PM7 method) of an H-type aggregate of 1. a) monomer, b) dimer side view, c) dimer topview, d) tetramer side view, e) tetramer top view.
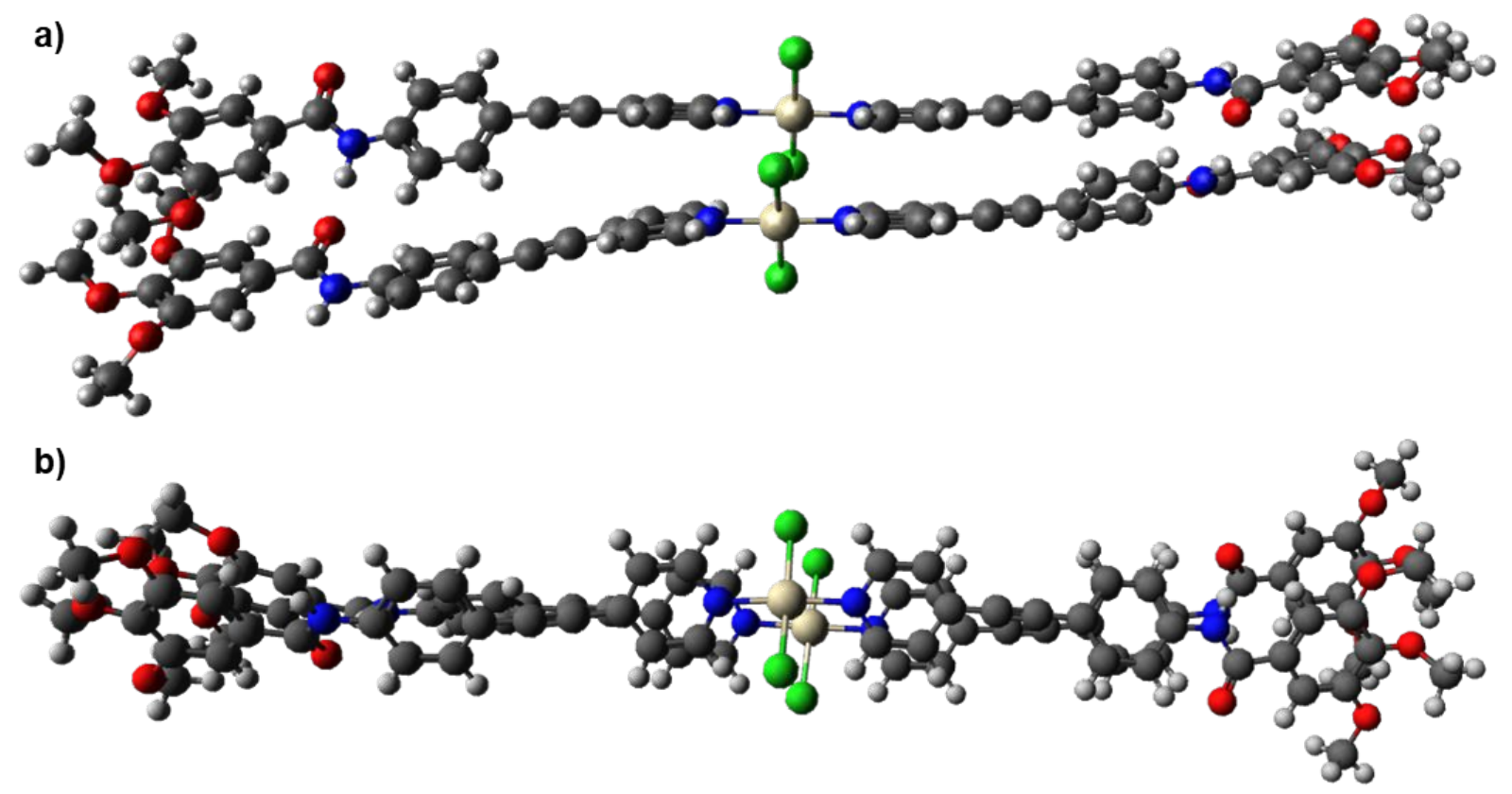

Figure S38. Geometry optimized structures (DFT) of a dimer of an H-type aggregate of 1-OMe. a) side view, b) top-view. 
a)

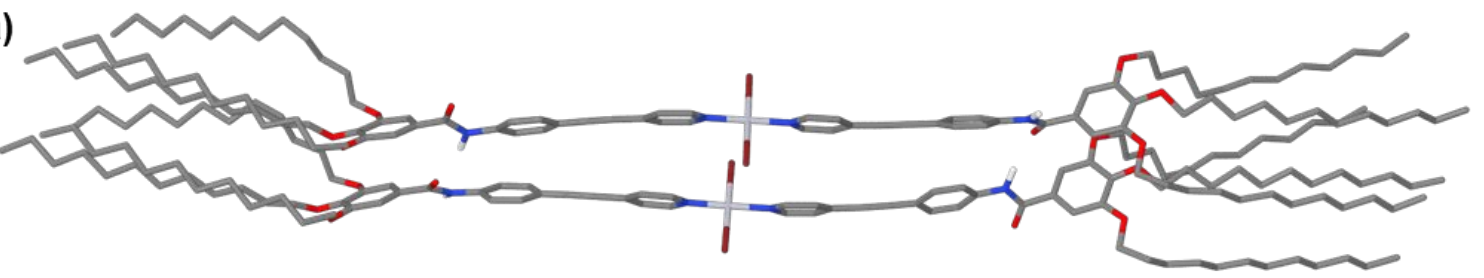

b)

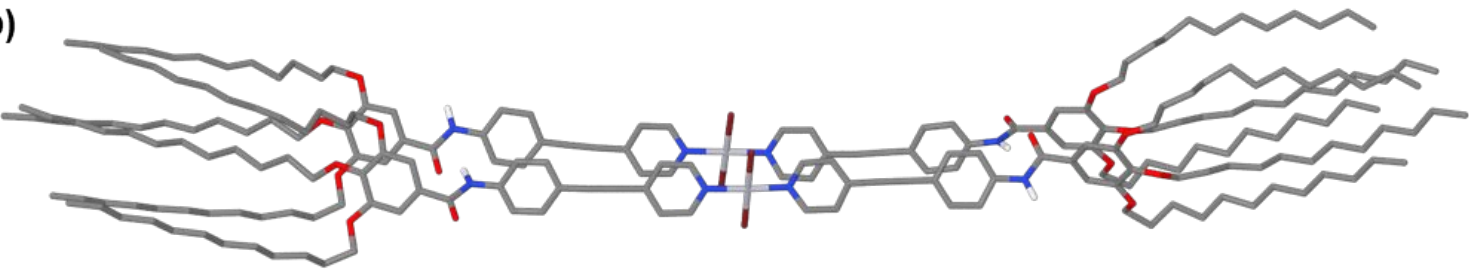

c)

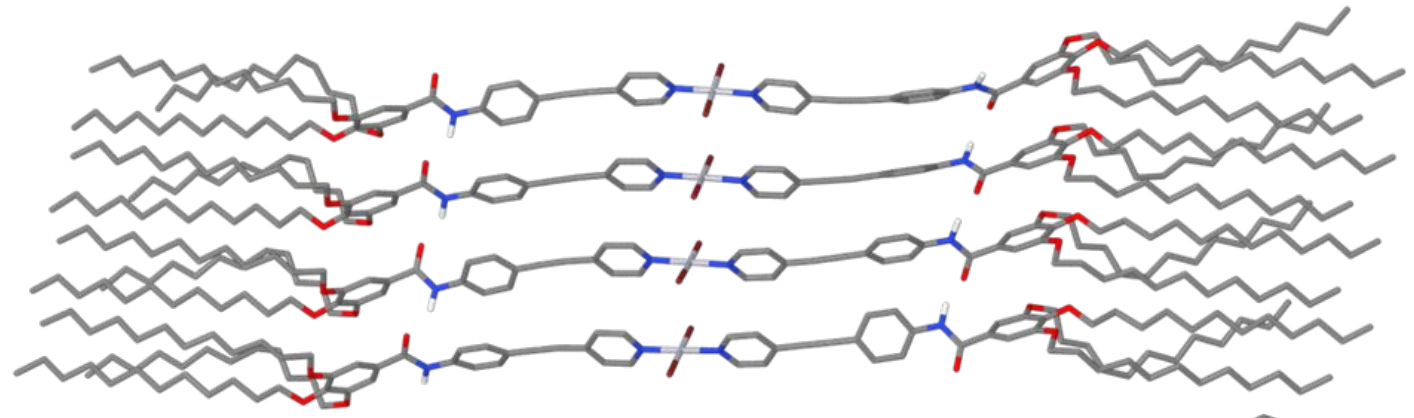

d)

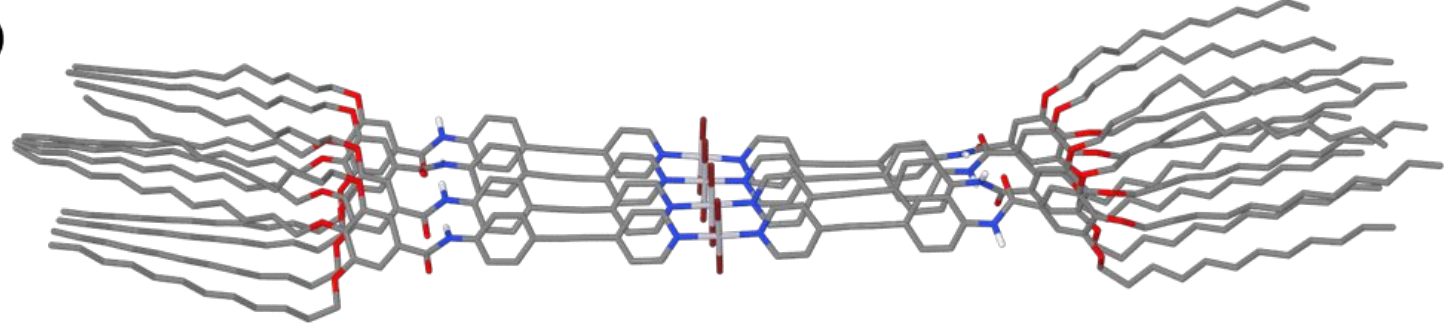

Figure S39. Geometry optimized structures (semiempirical PM7 method) of an H-type aggregate of 2. a) monomer, b) dimer side view, c) dimer topview, d) tetramer side view, e) tetramer top view.

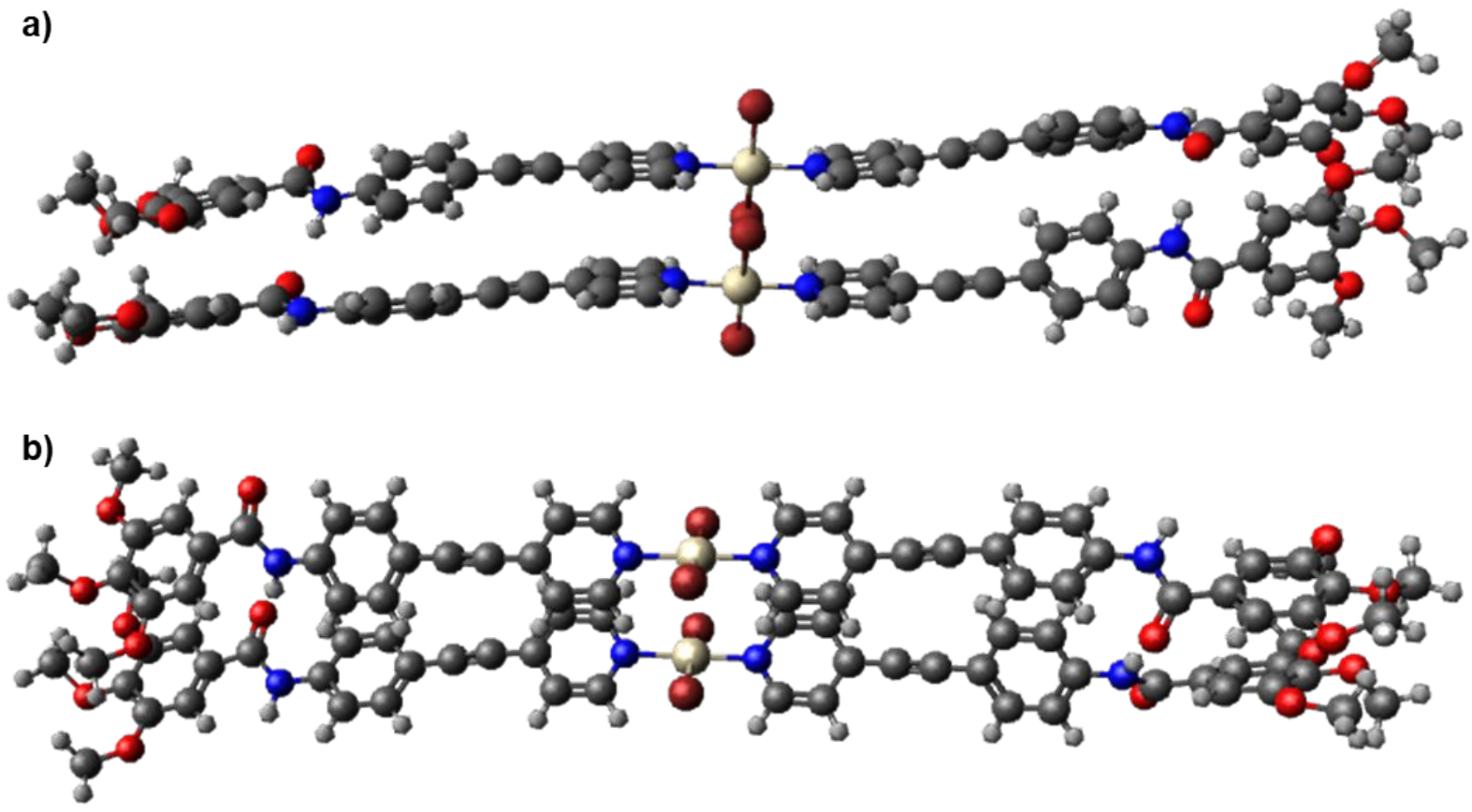

Figure S40. Geometry optimized structures (DFT) of a dimer of an H-type aggregate of 2-OMe. a) side view, b) top-view. 
a)

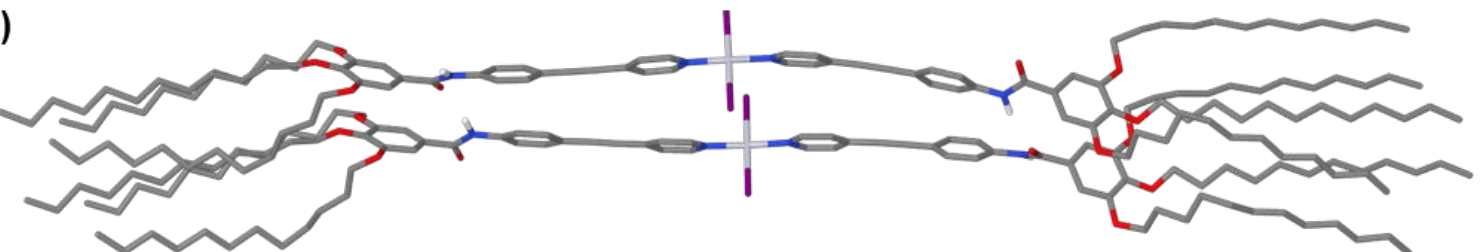

b)

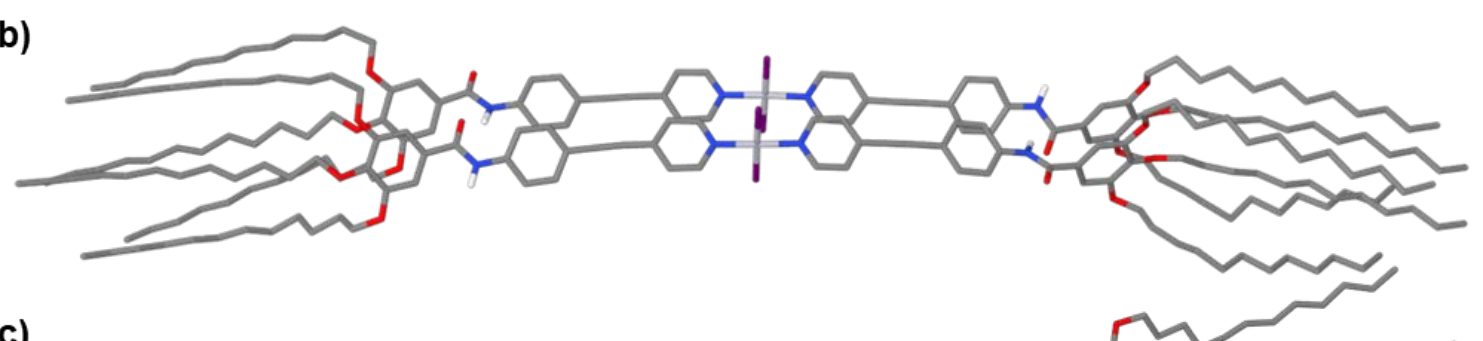

c)

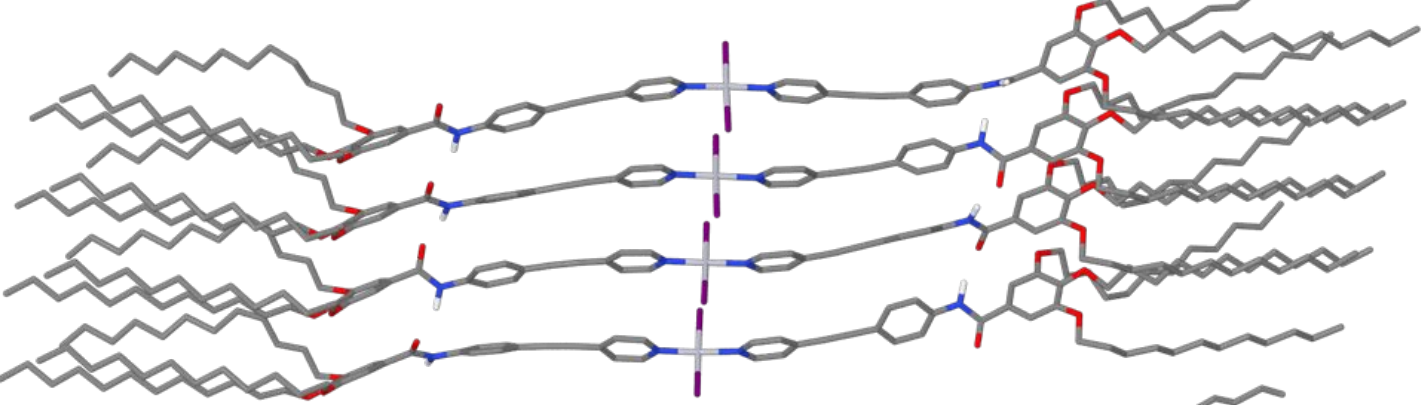

d)

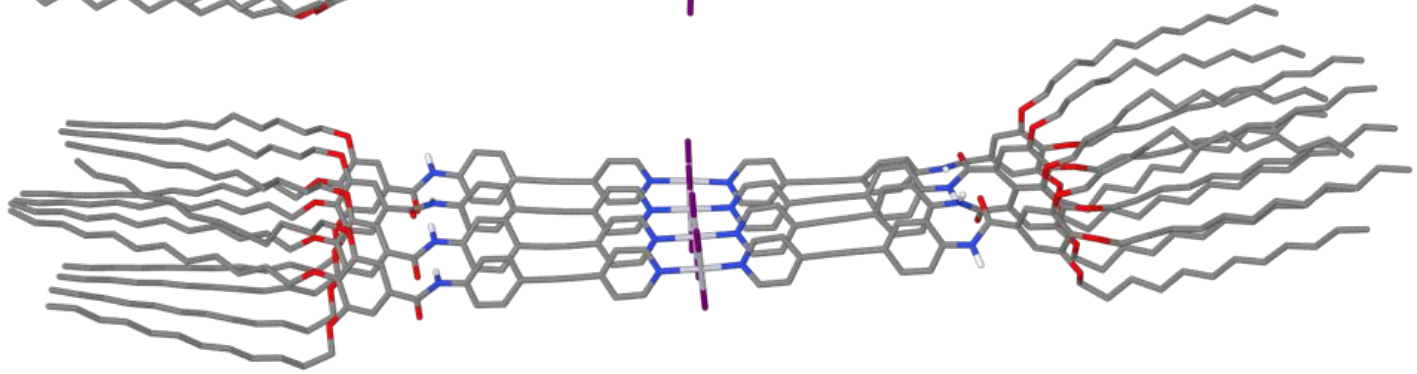

Figure S41. Geometry optimized structures (semiempirical PM7 method) of an H-type aggregate of 3. a) monomer, b) dimer side view, c) dimer topview, d) tetramer side view, e) tetramer top view.
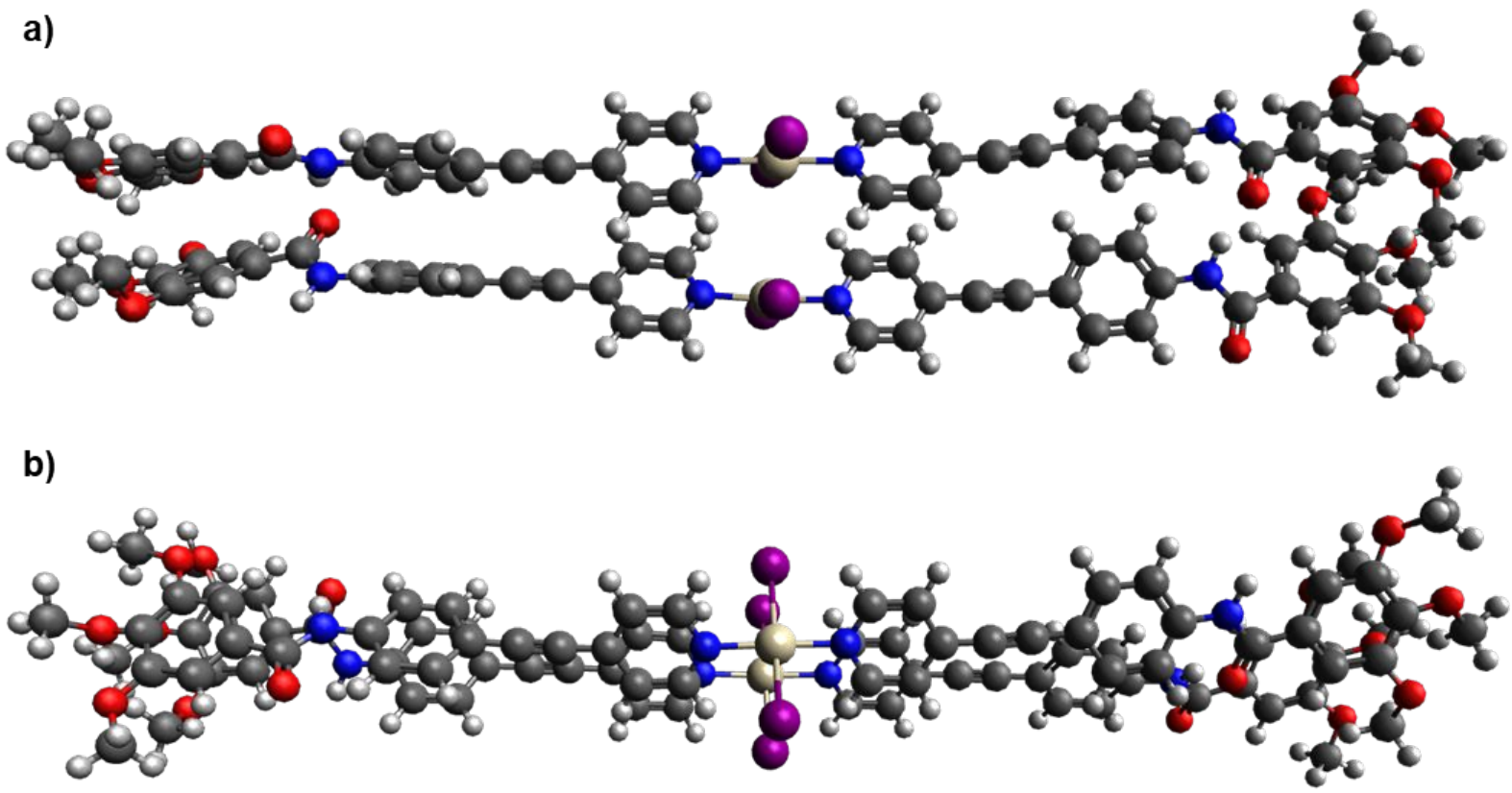

Figure S42. Geometry optimized structures (DFT) of a dimer of an H-type aggregate of 3-OMe. a) side view, b) top-view. 


\section{Experimental Section}

Materials and Methods

\section{General Procedures}

All solvents were dried according to standard procedures. Reagents were used as purchased. All air-sensitive reactions were carried out under argon or nitrogen atmosphere.

For all spectroscopic measurements, spectroscopic grade solvents were used.

\section{Colum Chromatography}

Preparative column chromatography was conducted in self-packed glass columns of different sizes with silica gel (Merck Silica 60, particle size $0.04-0.063 \mathrm{~nm})$.

\section{NMR measurements}

${ }^{1} \mathrm{H}$ and ${ }^{13} \mathrm{C}$ NMR spectra were recorded on a Bruker Avance II $300\left({ }^{1} \mathrm{H}: 300 \mathrm{MHz},{ }^{13} \mathrm{C}: 75 \mathrm{MHz}\right)$. and a Bruker Avance II $400\left({ }^{1} \mathrm{H}\right.$ : $400 \mathrm{MHz} ;{ }^{13} \mathrm{C}: 101 \mathrm{MHz}$ ). Additional $1 \mathrm{D}{ }^{1} \mathrm{H}$ as well as $2 \mathrm{D}$ spectra were recorded on an Agilent DD2 $500\left({ }^{1} \mathrm{H}: 500 \mathrm{MHz},{ }^{13} \mathrm{C}\right.$ : $126 \mathrm{MHz}$ ) and an Agilent DD2 $600\left({ }^{1} \mathrm{H}: 600 \mathrm{MHz},{ }^{13} \mathrm{C}: 151 \mathrm{MHz}\right)$ at a standard temperature of $298 \mathrm{~K}$ in deuterated solvents. The recorded spectra were referenced to the remaining resonance signals of the deuterated solvents. The coupling constant $\mathrm{J}$ of the measured spin multiplets is given in $\operatorname{Hertz}(\mathrm{Hz})$ and the chemical shift $\delta$ is given in reference to the chemical shift of trimethylsilane $(0 \mathrm{ppm})$. Multiplicities for proton signals are abbreviated as $s, d, t, q$ and $m$ for singlet, doublet, triplet, quadruplet and multiplet, respectively.

\section{Mass spectrometry (MS)}

MALDI mass spectra were recorded on a Bruker Daltronics Ultraflex ToF/ToF or a Bruker Daltronics Autoflex Speed with a SmartBeamTM NdYAF-Laser with a wavelength of $335 \mathrm{~nm}$. ESI mass spectra were measured on a Bruker MicrOToF system. The signals are described by their mass/charge ratio $(\mathrm{m} / \mathrm{z})$ in $\mathrm{u}$.

\section{UV-Vis spectroscopy}

UV-Vis absorption spectra were recorded on a JASCO $\mathrm{V}$-770 or a JASCO $\mathrm{V}-750$ with a spectral bandwidth of $1.0 \mathrm{~nm}$ and a scan rate of $1000 \mathrm{~nm} \mathrm{~min}^{-1}$ or on an Agilent Cary 4000 with a spectral bandwidth of $2 \mathrm{~nm}$ at a scan rate of $600 \mathrm{~nm} \mathrm{~min}{ }^{-1}$. Glass cuvettes with an optical length of $1 \mathrm{~cm}, 1 \mathrm{~mm}$ and $0.1 \mathrm{~mm}$ and $0.01 \mathrm{~mm}$ were used. All measurements were conducted in commercially available solvents in spectroscopic grade.

\section{FT-IR spectroscopy}

Solution and solid-state measurements were carried out using a JASCO-FT-IR-6800 equipped with a CaF 2 cell with a path length of $0.1 \mathrm{~mm}$.

\section{Atomic force microscopy (AFM)}

The AFM images were recorded on a Multimode ${ }^{\circledR} 8$ SPM System manufactured by Bruker AXS. The used cantilevers were AC200TS by Oxford Instruments with an average spring constant of $9 \mathrm{~N} \mathrm{~m}^{-1}$, an average frequency of $150 \mathrm{kHz}$, an average length of $200 \mu \mathrm{m}$, an average width of $40 \mu \mathrm{m}$ and an average tip radius of $7 \mathrm{~nm}$. All samples were drop casted or spin-coated at a spin velocity between 2000 and $4000 \mathrm{rpm}$ from freshly prepared solutions onto an HOPG surface:

\section{Dynamic Light Scattering (DLS)}

DLS spectra were been recorded on a CGS-3 Compact Goniometer System manufactured by $A L V$ GmbH, equipped with a HeNe laser with a wavelength of $632.8 \mathrm{~nm}(22 \mathrm{~mW})$ and an ALV/LSE-5004 Digital Correlator by ALV GmbH.

\section{Gel permeation chromatography (GPC)}

Gel permeation chromatography was performed on a Shimadzu prominence GPC system equipped with two Tosoh TSKgel columns (G2500H XL; $7.8 \mathrm{~mm}$ I.D. x $30 \mathrm{~cm}, 5 \mu \mathrm{m}$; Part. No. 0016135) with $\mathrm{CH}_{2} \mathrm{Cl}_{2}$ as eluent. The solvent flow was set to be $1 \mathrm{~mL} / \mathrm{min}$. Detection was carried out via a Shimadzu prominence SPD-M20A diode array detector (DAD). 


\section{Synthesis and Characterization}

The pyridine-based oligo(phenylene-ethynylene) (OPE) ligand with an amide-appended peripheral solubilizing moiety was synthesized according to previously published protocols involving a series of deprotection and coupling reactions (Scheme S1). ${ }^{10,11}$ Complex 1 bearing chloride ligands was synthesized according to a standard complexation protocol from the corresponding pyridine-OPE ligand and bis(benzonitrile)dichloroplatinum(II) in toluene at $110^{\circ} \mathrm{C} .{ }^{3}$ Due to the lack of similar commercial precursors bearing other halogens, 2 and 3 were synthesized starting from $\mathrm{PtBr}_{2}$ and $\mathrm{PtI}_{2}, \mathrm{respectively}_{\text {In a first }}$ step the inorganic compounds were converted into soluble complexes by treatment with tetrahydrothiophene in DCM. ${ }^{12}$ Subsequently, these precursor-complexes were reacted with the pyridine-OPE ligand in toluene at $110{ }^{\circ} \mathrm{C}$, yielding complexes 2 and 3. Initial efforts to synthesize an analogous fluorine-substituted complex were unsuccessful. This is not surprising, considering the scarce reports on $\mathrm{Pd}^{\mathrm{II}}$ and $\mathrm{Pt}^{\mathrm{II}}$ difluoride complexes. ${ }^{13-15}$ Commonly it is assumed that the compounds are hard to access due to a variety of reasons, but often this complex matter is reduced to the unfavorable interactions between the soft $\mathrm{Pt}^{\mathrm{II}}$ metal center and the hard fluoride anion. ${ }^{16-19}$

Scheme S1. Synthesis of the title compounds 1-3.

Synthesis of complex-precursors

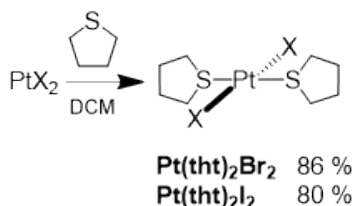

Synthesis of the ligand and complexes 1-3

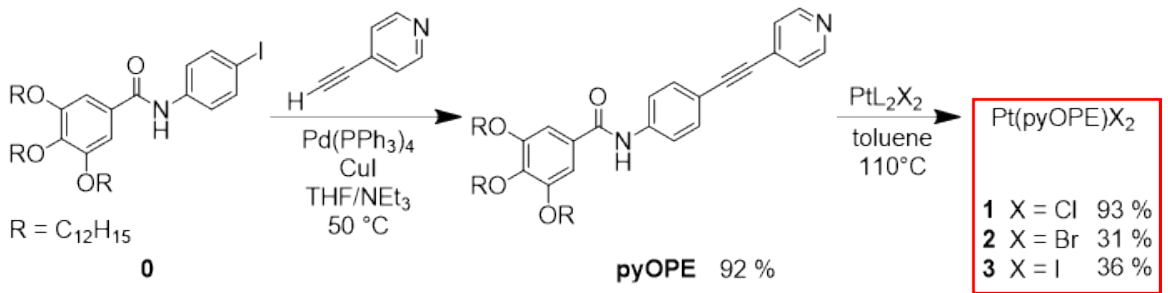

$\mathbf{P t}(\mathbf{t h t})_{2} \mathbf{I}_{2},{ }^{12} \mathrm{~N}$-(4-iodophenyl)-3,4,5-tridodecyloxy benzamide $\mathbf{0}^{10}$ and 4-ethynylpyridine ${ }^{11}$ were prepared following reported synthetic procedures modifications thereof and showed identical spectroscopic properties to those reported.

\section{Synthesis of the complex precursors $\mathrm{Pt}(\text { (tht })_{2} \mathrm{X}_{2}$}

$\mathrm{PtX}_{2}$ (1 eq) was suspended in dry DCM and tetrahydrothiophene (tht, 4 eq) was added. The reaction mixture was stirred at room temperature for 18 hours. The reaction solution was filtered through a syringe filter (teflon, $0.2 \mu \mathrm{M}$ ) to remove residual $\mathrm{PtX}_{2}$ and the filter was rinsed with DCM. The solvent was removed in vacuo and the product was washed with pentane.

\section{$\operatorname{Pt}(\text { tht })_{2} \mathrm{Br}_{2}$}

${ }^{1} \mathrm{H}$ NMR $\left(\mathrm{CDCl}_{3}, 400 \mathrm{MHz}\right) \delta(\mathrm{ppm})=3.76\left(\mathrm{~s}, \mathrm{br}, 4 \mathrm{H}, \mathrm{S}-\mathrm{CH}_{2}\right), 2.93\left(\mathrm{~s}, \mathrm{br}, 4 \mathrm{H}, \mathrm{S}-\mathrm{CH}_{2}\right), 2.22\left(\mathrm{~s}, \mathrm{br}, 4 \mathrm{H}, \mathrm{S}-\mathrm{CH}_{2}-\mathrm{CH}_{2}\right), 2.03(\mathrm{~s}, \mathrm{br}, 4 \mathrm{H}$, $\mathrm{S}-\mathrm{CH}_{2}-\mathrm{CH}_{2}$ ). Two sets of signals due to cis/trans mixture.

${ }^{13}$ C NMR $\left(75 \mathrm{MHz}, \mathrm{CDCl}_{3}\right) \delta(\mathrm{ppm})=39.08,29.98$. 


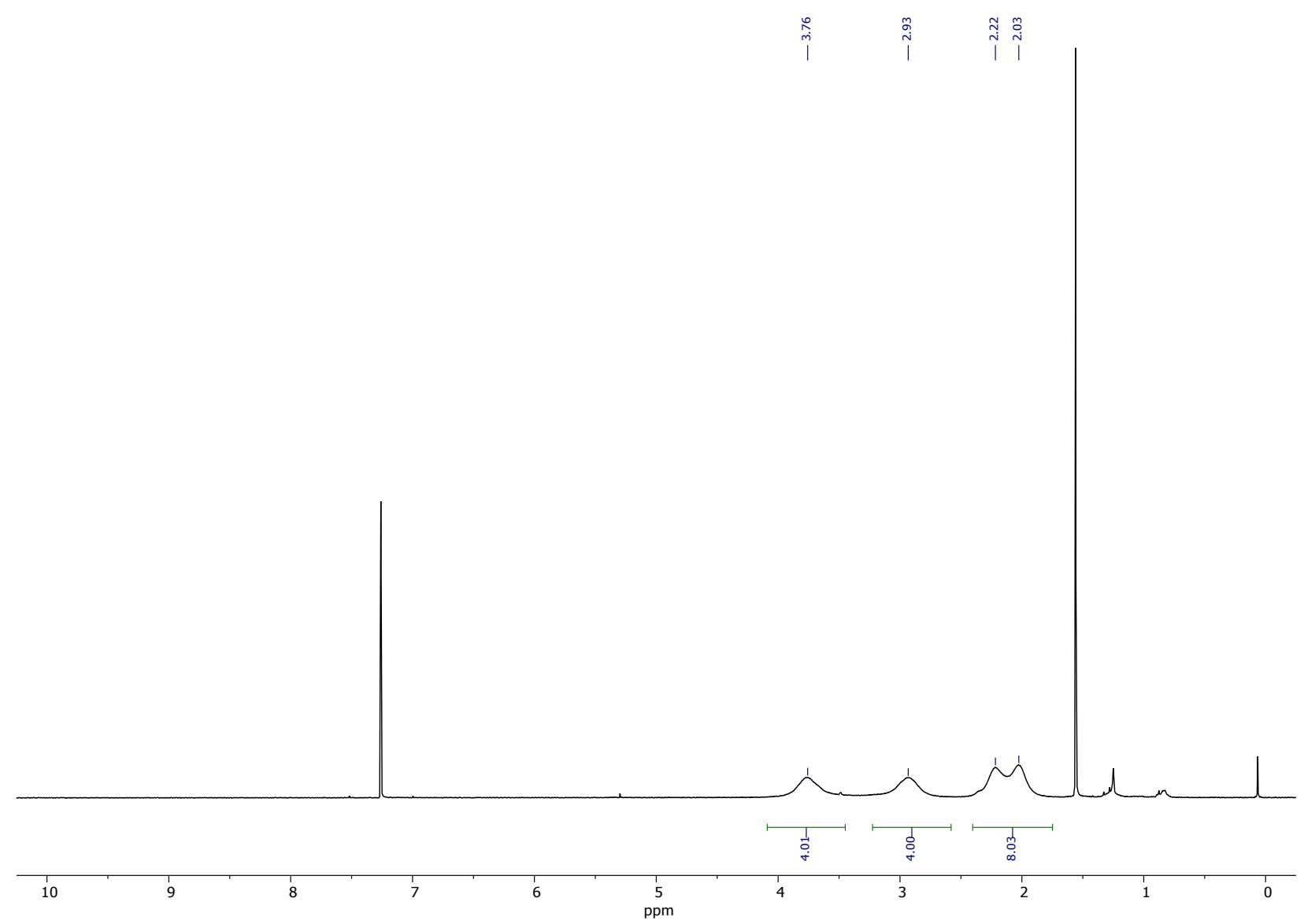

Figure S43. ${ }^{1} \mathrm{H}$ NMR $\left(\mathrm{CDCl}_{3}, 400 \mathrm{MHz}\right)$ spectrum of complex precursor $\mathbf{P t}(\mathbf{t h t})_{2} \mathbf{B r}_{2}$.

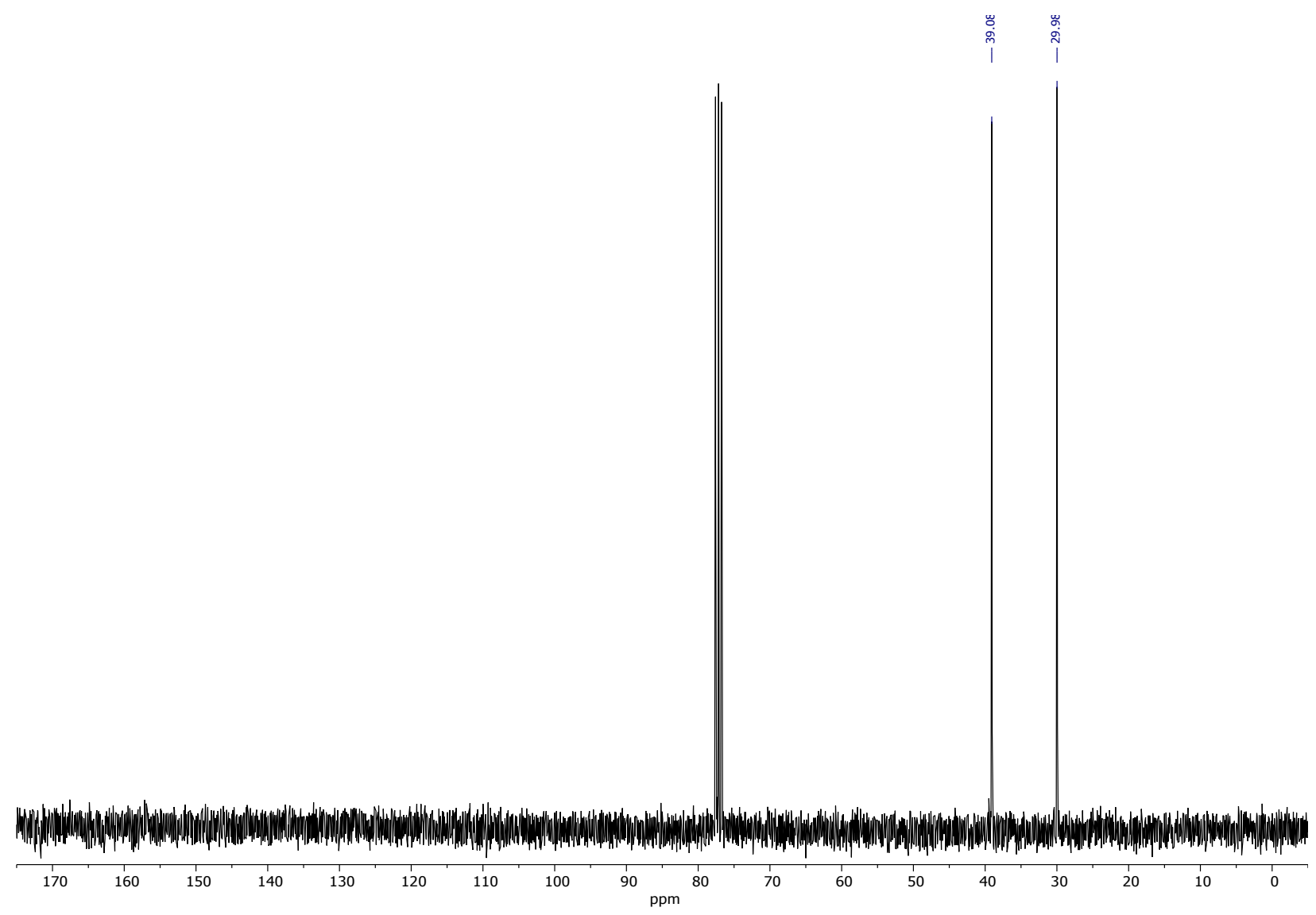

Figure S44. ${ }^{13} \mathrm{C}$ NMR $\left(\mathrm{CDCl}_{3}, 75 \mathrm{MHz}\right)$ spectrum of complex precursor $\mathbf{P t}(\text { tht })_{2} \mathbf{B r}_{2}$. 


\section{$\operatorname{Pt}(\text { tht })_{2} \mathbf{I}_{2}$}

${ }^{1} \mathrm{H}$ NMR $\left(\mathrm{CDCl}_{3}, 300 \mathrm{MHz}\right) \delta(\mathrm{ppm})=3.48\left(\mathrm{~s}, \mathrm{br}, 4 \mathrm{H}, \mathrm{S}-\mathrm{CH}_{2}\right), 2.11\left(\mathrm{~s}, \mathrm{br}, 4 \mathrm{H}, \mathrm{S}-\mathrm{CH}_{2}-\mathrm{CH}_{2}\right)$.

${ }^{13} \mathrm{C} \mathrm{NMR}\left(101 \mathrm{MHz}, \mathrm{CDCl}_{3}\right) \delta(\mathrm{ppm})=42.65,29.88$.

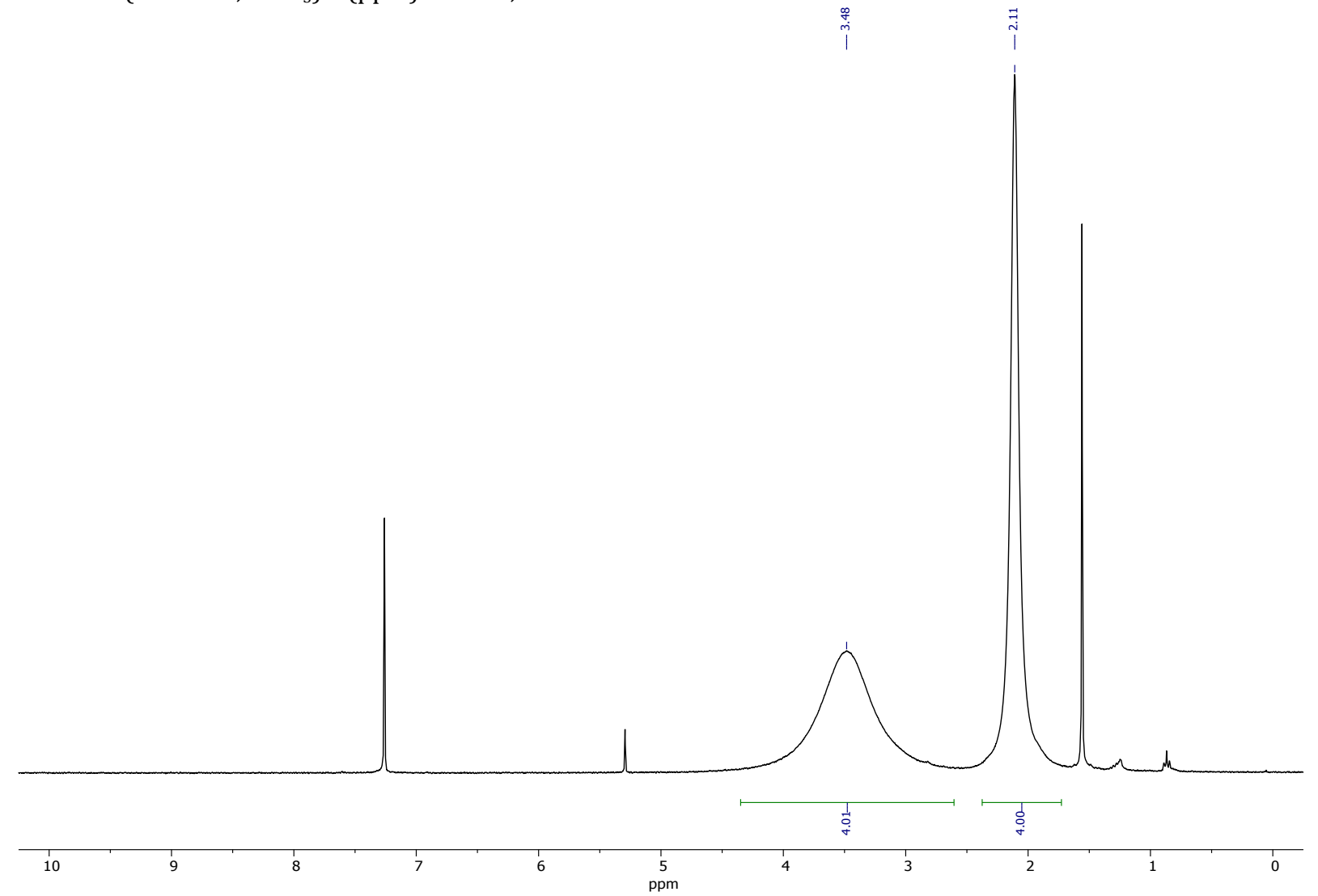

Figure S45. ${ }^{1} \mathrm{H}$ NMR $\left(\mathrm{CDCl}_{3}, 300 \mathrm{MHz}\right)$ spectrum of complex precursor $\mathbf{P t}(\mathbf{t h t})_{2} \mathbf{I}_{2}$.

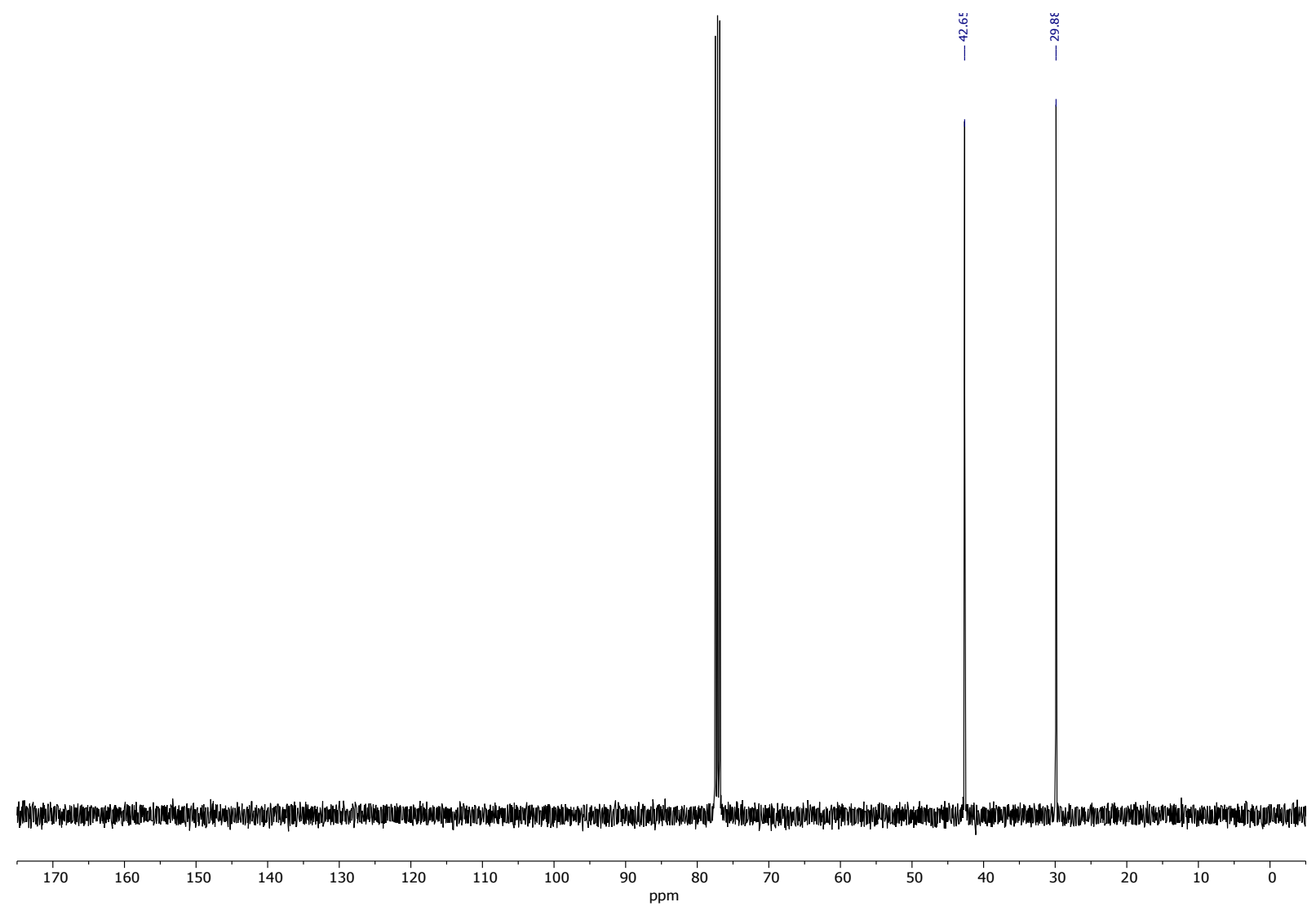

Figure S46. ${ }^{13} \mathrm{C}$ NMR $\left(\mathrm{CDCl}_{3}, 101 \mathrm{MHz}\right)$ spectrum of complex precursor $\mathbf{P t}(\mathbf{t h t})_{2} \mathbf{I}_{2}$. 
$\mathrm{N}$-(4-iodophenyl)-3,4,5-tridodecyloxy benzamide $\mathbf{0}$ (1 eq), tetrakis(triphenylphosphine)palladium(0) (0.05 eq) and copper(I) iodide (0.03 eq) were dissolved in a 1:1 mixture of degassed THF:NEt $\mathrm{T}_{3}$. The solution was stirred at $50{ }^{\circ} \mathrm{C}$ for 30

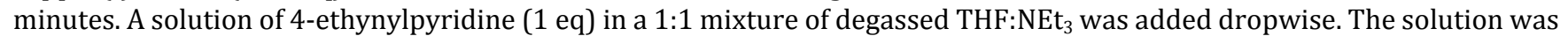
stirred at $50{ }^{\circ} \mathrm{C}$ for 18 hours. The solvents were removed in vacuo and the residue was dissolved in DCM and filtered through a thin layer of Celite $545^{\circledR}$. The solvent was removed in vacuo and the crude product was purified by column chromatography (DCM:EtOAc 20:1 $\rightarrow$ 10:1) yielding the product as a colorless solid (94\%).

${ }^{1} \mathbf{H}$ NMR $\left(\mathrm{CDCl}_{3}, 600 \mathrm{MHz}\right): \delta(\mathrm{ppm})=8.60\left(\mathrm{AA}^{\prime} \mathrm{XX}\right.$ ', 2H, $\left.\mathrm{H}_{1}\right), 7.86\left(\mathrm{~s}, 1 \mathrm{H}, \mathrm{H}_{5}\right), 7.68\left(\mathrm{~m}, 2 \mathrm{H}, \mathrm{H}_{4}\right), 7.56\left(\mathrm{~m}, 2 \mathrm{H}, \mathrm{H}_{3}\right), 7.37\left(\mathrm{AA}^{\prime} \mathrm{XX}\right.$, $\left.2 \mathrm{H}, \mathrm{H}_{2}\right), 7.04\left(\mathrm{~s}, 2 \mathrm{H}, \mathrm{H}_{6}\right), 4.02\left(\mathrm{~m}, 6 \mathrm{H}, \mathrm{OCH}_{2}\right), 1.82\left(\mathrm{~m}, 4 \mathrm{H}, \mathrm{C}^{2} \mathrm{H}_{2}^{\mathrm{a}}\right), 1.75\left(\mathrm{~m}, 2 \mathrm{H}, \mathrm{C}^{2} \mathrm{H}_{2}^{\mathrm{b}}\right), 1.47\left(\mathrm{~m}, 6 \mathrm{H}, \mathrm{C}^{3} \mathrm{H}_{2}\right), 1.31\left(\mathrm{~m}, 48 \mathrm{H}, \mathrm{C}^{4} \mathrm{H}_{2}-\right.$ $\left.\mathrm{C}^{11} \mathrm{H}_{2}\right), 0.88\left(\mathrm{~m}, 9 \mathrm{H}, \mathrm{CH}_{3}\right)$.

${ }^{13}$ C NMR $\left(\mathrm{CDCl}_{3}, 151 \mathrm{MHz}\right): \delta(\mathrm{ppm})=165.78,153.47,149.92,142.01,139.08,133.00,131.69,129.64,125.61,119.91,117.88$, 106.07, 93.99, 86.68, 73.75, 69.69, 32.09, 32.08, 30.48, 29.91, 29.89, 29.88, 29.85, 29.81, 29.79, 29.73, 29.56, 29.54, 29.52, 26.23, 22.85, 22.84, 14.26 . HR-MS (ESI-TOF, MeOH): calculated for $\mathrm{C}_{56} \mathrm{H}_{87} \mathrm{~N}_{2} \mathrm{O}_{4}{ }^{+}[\mathrm{M}+\mathrm{H}]^{+}:$
found:

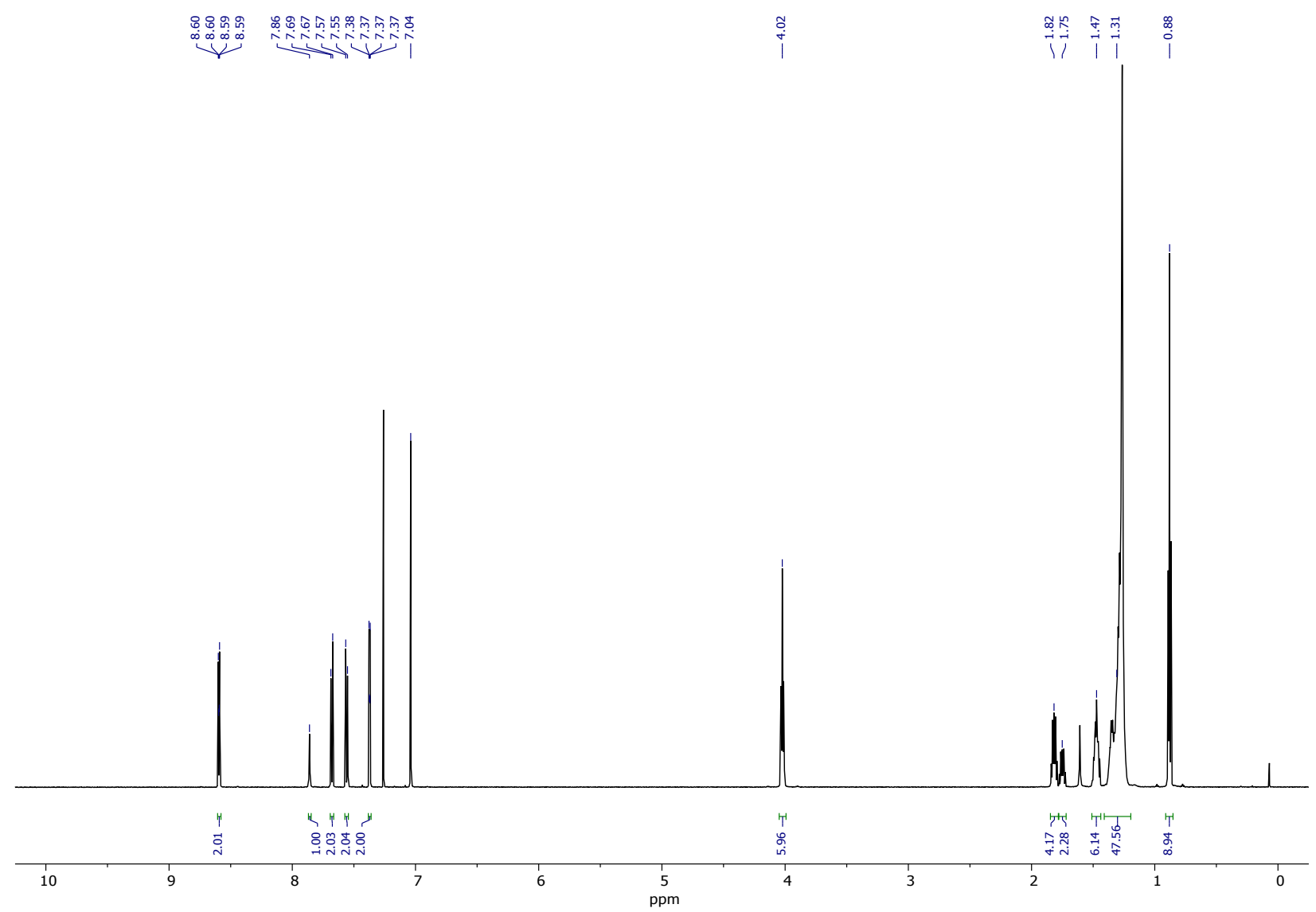

Figure S47. ${ }^{1} \mathrm{H} \mathrm{NMR}\left(\mathrm{CDCl}_{3}, 600 \mathrm{MHz}\right)$ spectrum of ligand pyOPE. 


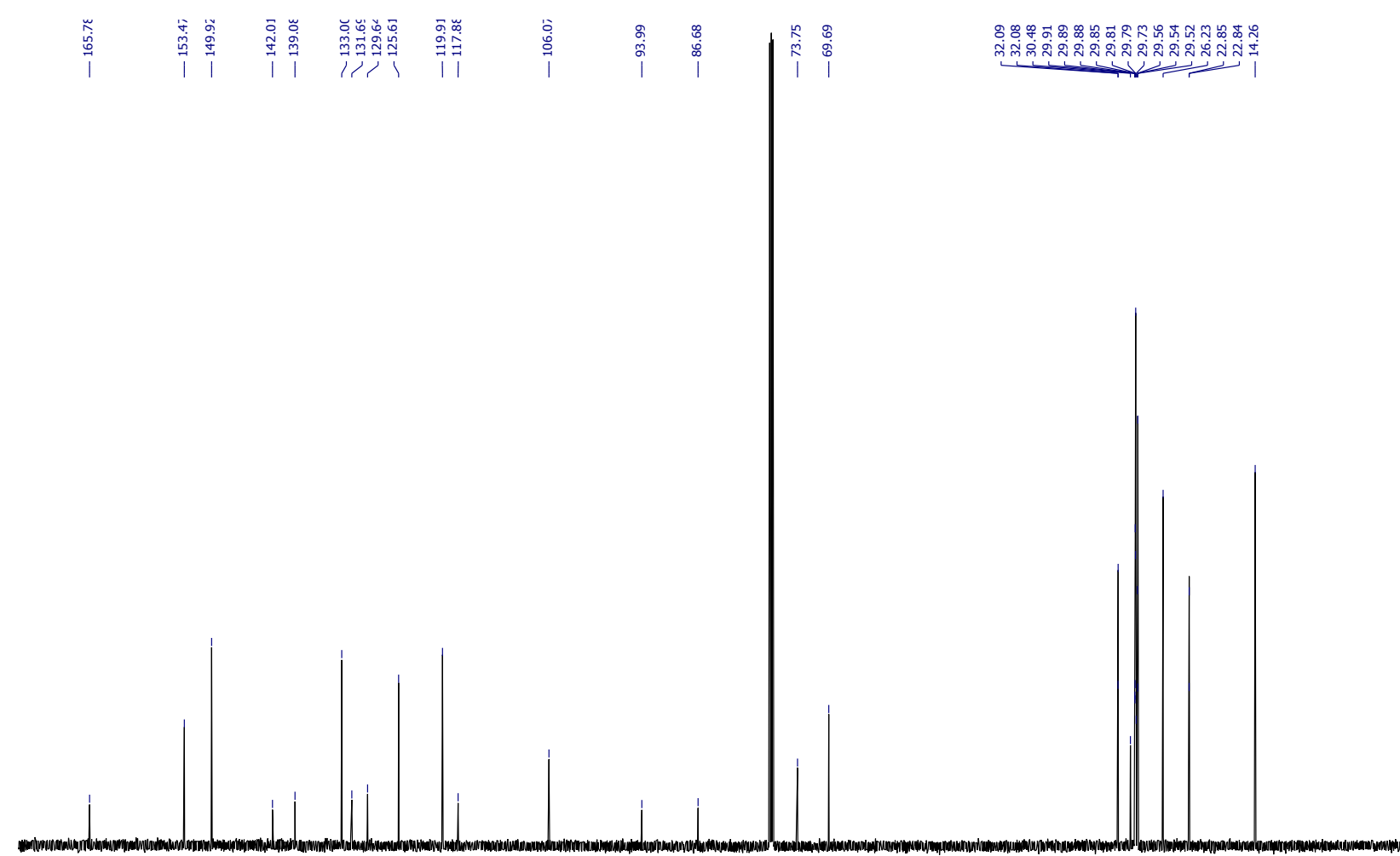

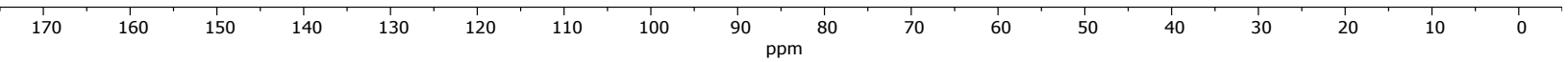

Figure S48. ${ }^{13} \mathrm{C}$ NMR $\left(\mathrm{CDCl}_{3}, 151 \mathrm{MHz}\right)$ spectrum of compound pyOPE.

\section{Synthesis of complex 1}

Ligand (pyOPE) (500 mg, 587 $\mu \mathrm{mmol}, 2 \mathrm{eq})$ and $\mathrm{Pt}(\mathrm{PhCN})_{2} \mathrm{Cl}_{2}(139 \mathrm{mg}, 294 \mu \mathrm{mol}, 1 \mathrm{eq})$ were placed in pressure tube and were subjected to five vacuum/Ar cycles. Subsequently, $5 \mathrm{~mL}$ of dry, degassed toluene were added, the tube was sealed and the reaction mixture was stirred at $110^{\circ} \mathrm{C}$ for one week. The solvents were evaporated and the resulting crude solid was purified first by column chromatography (DCM:EtOAc 20:1 $\rightarrow$ 10:1) and finally by size exclusion chromatography. The title compound was obtained as a pale-yellow solid (540 mg, $274 \mu \mathrm{mol}, 93 \%$ ).

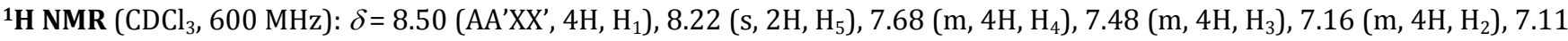
$\left(\mathrm{s}, 4 \mathrm{H}, \mathrm{H}_{6}\right), 4.01\left(\mathrm{~m}, 12 \mathrm{H}, \mathrm{OCH}_{2}\right), 1.78\left(\mathrm{~m}, 12 \mathrm{H}, \mathrm{C}^{2} \mathrm{H}_{2}\right), 1.46\left(\mathrm{~m}, 12 \mathrm{H}, \mathrm{C}^{3} \mathrm{H}_{2}\right), 1.30\left(\mathrm{~m}, 96 \mathrm{H}, \mathrm{C}^{4} \mathrm{H}_{2}-\mathrm{C}^{11} \mathrm{H}_{2}\right), 0.88\left(\mathrm{~m}, 18 \mathrm{H}, \mathrm{CH}_{3}\right)$.

${ }^{13} \mathrm{C} \mathrm{NMR}\left(\mathrm{CDCl}_{3}, 151 \mathrm{MHz}\right): \delta(\mathrm{ppm})=165.67,153.19,152.60,141.61,139.87,134.20,133.09,129.30,126.65,119.55,116.56$, $106.01,98.53,85.44,73.49,69.38,31.93,31.92,30.36,29.75,29.74,29.70,29.70,29.66,29.65,29.58,29.42,29.39,29.36$, $29.35,26.10,26.08,22.68,14.11,14.10$.

HR-MS (ESI-Orbitrap, $\mathrm{MeOH} / \mathrm{CHCl}_{3}$ ): $\quad$ calculated for $\mathrm{C}_{112} \mathrm{H}_{172} \mathrm{~N}_{4} \mathrm{O}_{8} \mathrm{Cl}_{2} \mathrm{PtNa}^{+}[\mathrm{M}+\mathrm{Na}]^{+}$: found: 1991.2109 


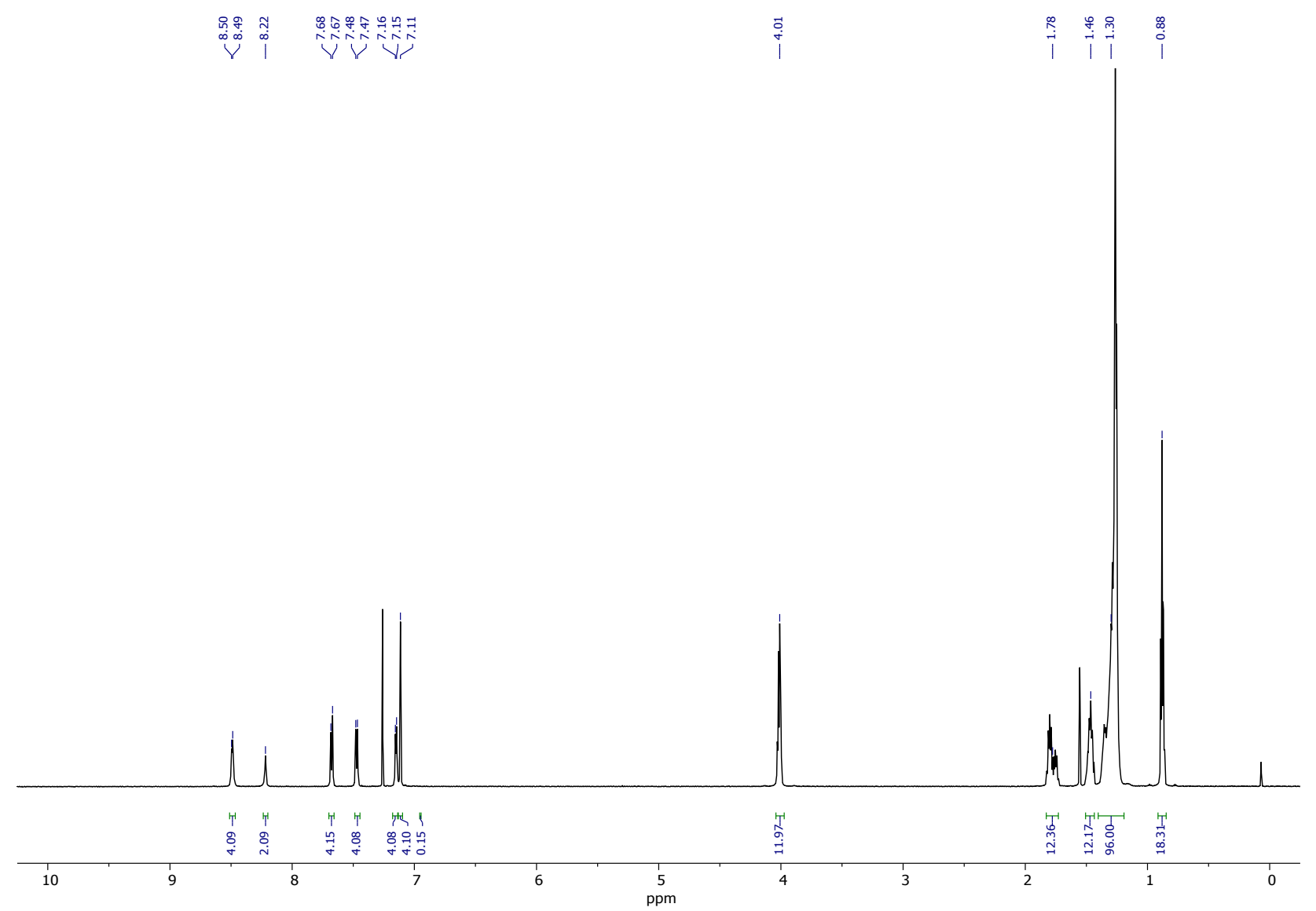

Figure S49. ${ }^{1} \mathrm{H}$ NMR $\left(\mathrm{CDCl}_{3}, 600 \mathrm{MHz}\right)$ spectrum of compound 1.

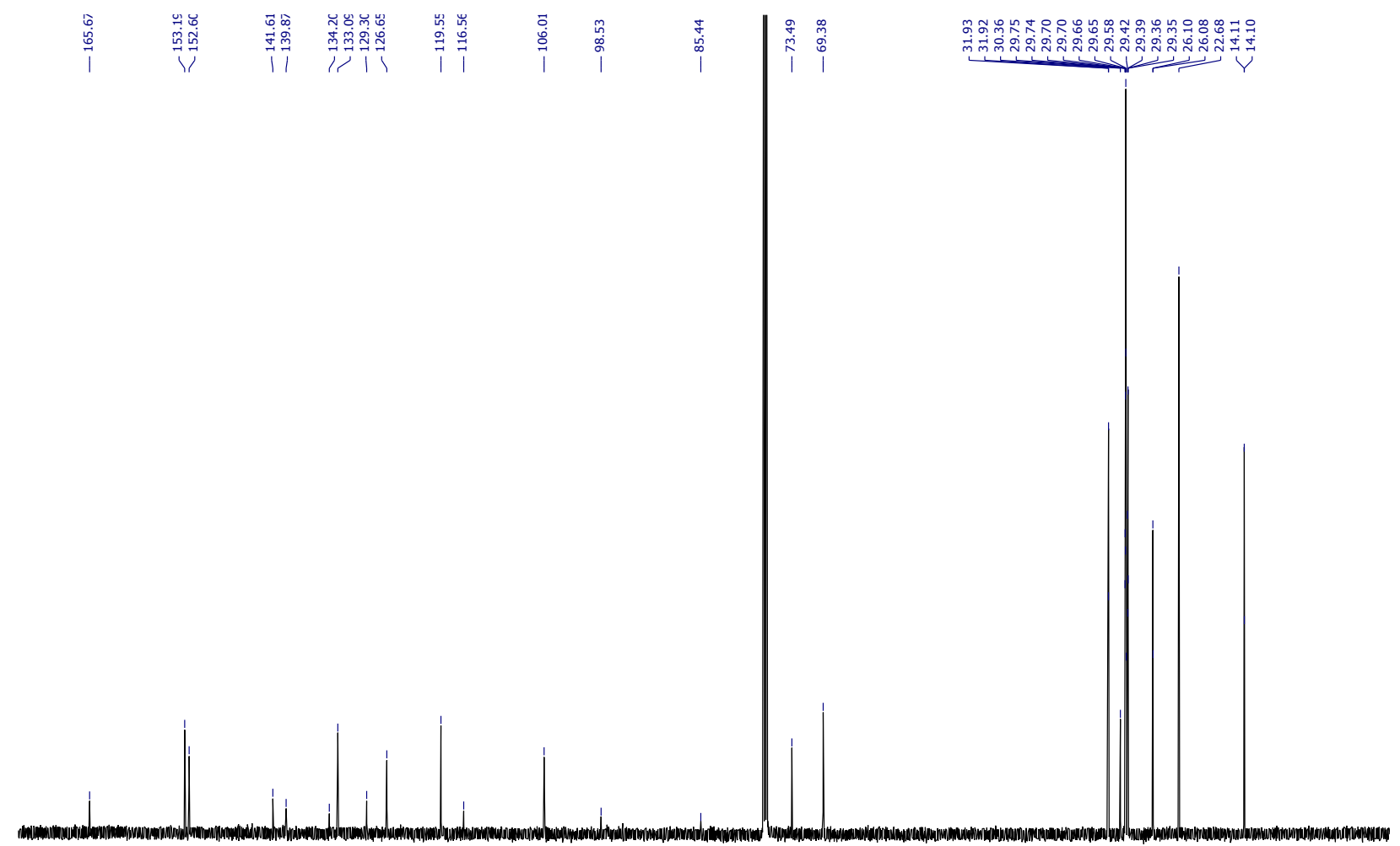

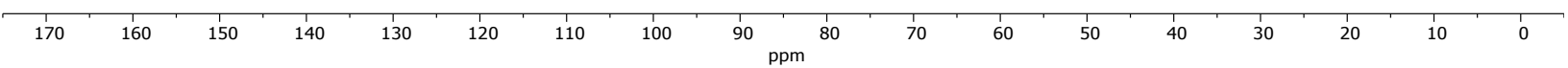

Figure S50. ${ }^{13} \mathrm{C}$ NMR $\left(\mathrm{CDCl}_{3}, 151 \mathrm{MHz}\right)$ spectrum of compound $\mathbf{1}$. 


\section{Synthesis of complexes $2+3$}

Ligand (pyOPE) (2 eq) and Pt(tht) $)_{2} \mathrm{X}_{2}$ (1 eq) were placed in a pressure tube and were subjected to five vacuum/Ar cycles. Subsequently, $3 \mathrm{~mL}$ of dry, degassed toluene were added and the mixture was heated to $110^{\circ} \mathrm{C}$ and stirred at this temperature for one week. The brown reaction-solution was brought to room temperature and cooled in a freezer for $1 \mathrm{~h}$, resulting in the precipitation of a yellow/brown solid. The cold solution was filtered and the solid residue was washed several times with cold toluene, cold DCM and cold pentane. The solid was dried in vacuo yielding the product as a bright yellow (2) or orange (3) powder.

Table S7. Experimental conditions and yields for compounds $2(X=B r)$ and $\mathbf{3}(\mathrm{X}=\mathrm{I})$ :

\begin{tabular}{|l|l|l|}
\hline Compound & ${\text { Scale }[\boldsymbol{\mu m o l}]^{*}}^{*}$ & Yield [\%] \\
\hline $\mathbf{2}$ & 94 & 31 \\
\hline $\mathbf{3}$ & 80 & 36 \\
\hline
\end{tabular}

*The scale is given with reference to $\mathrm{Pt}(\mathrm{tht})_{2} \mathrm{X}_{2}(1 \mathrm{eq})$.

\section{Characterization of complex 2}

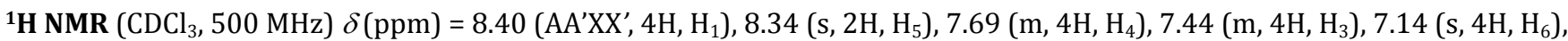
$7.05\left(\mathrm{AA}^{\prime} \mathrm{XX}, 4 \mathrm{H}, \mathrm{H}_{2}\right), 4.04\left(\mathrm{~m}, 12 \mathrm{H}, \mathrm{OCH}_{2}\right) 1.78\left(\mathrm{~m}, 12 \mathrm{H}, \mathrm{C}^{2} \mathrm{H}_{2}\right), 1.35\left(\mathrm{~m}, 108 \mathrm{H}, \mathrm{C}^{3} \mathrm{H}_{2}-\mathrm{C}^{11} \mathrm{H}_{2}\right), 0.88\left(\mathrm{~m}, 18 \mathrm{H}, \mathrm{CH}_{3}\right)$.

${ }^{13} \mathrm{C}$ NMR $\left(126 \mathrm{MHz}, \mathrm{CDCl}_{3}\right) \delta(\mathrm{ppm})=165.66,153.84,153.28,141.75,139.77,133.99,133.13,129.36,126.74,119.62,116.69$, 106.03 , 73.55, 69.48, 31.95, 31.93, 30.36, 29.76, 29.76, 29.75, 29.71, 29.71, 29.67, 29.66, 29.59, 29.42, 29.41, 29.40, 29.37, 29.36, 26.10, 26.09, 22.70, 14.12 .

HR-MS (ESI-Orbitrap, $\mathrm{MeOH} / \mathrm{CHCl}_{3}$ ):

calculated for $\mathrm{C}_{112} \mathrm{H}_{172} \mathrm{~N}_{4} \mathrm{O}_{8} \mathrm{Br}_{2} \mathrm{PtH}_{2}{ }^{2+}[\mathrm{M}+2 \mathrm{H}]^{2+}$ :

1029.56725

found: 1029.56857

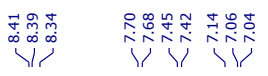

\section{$\stackrel{8}{+\infty}$}
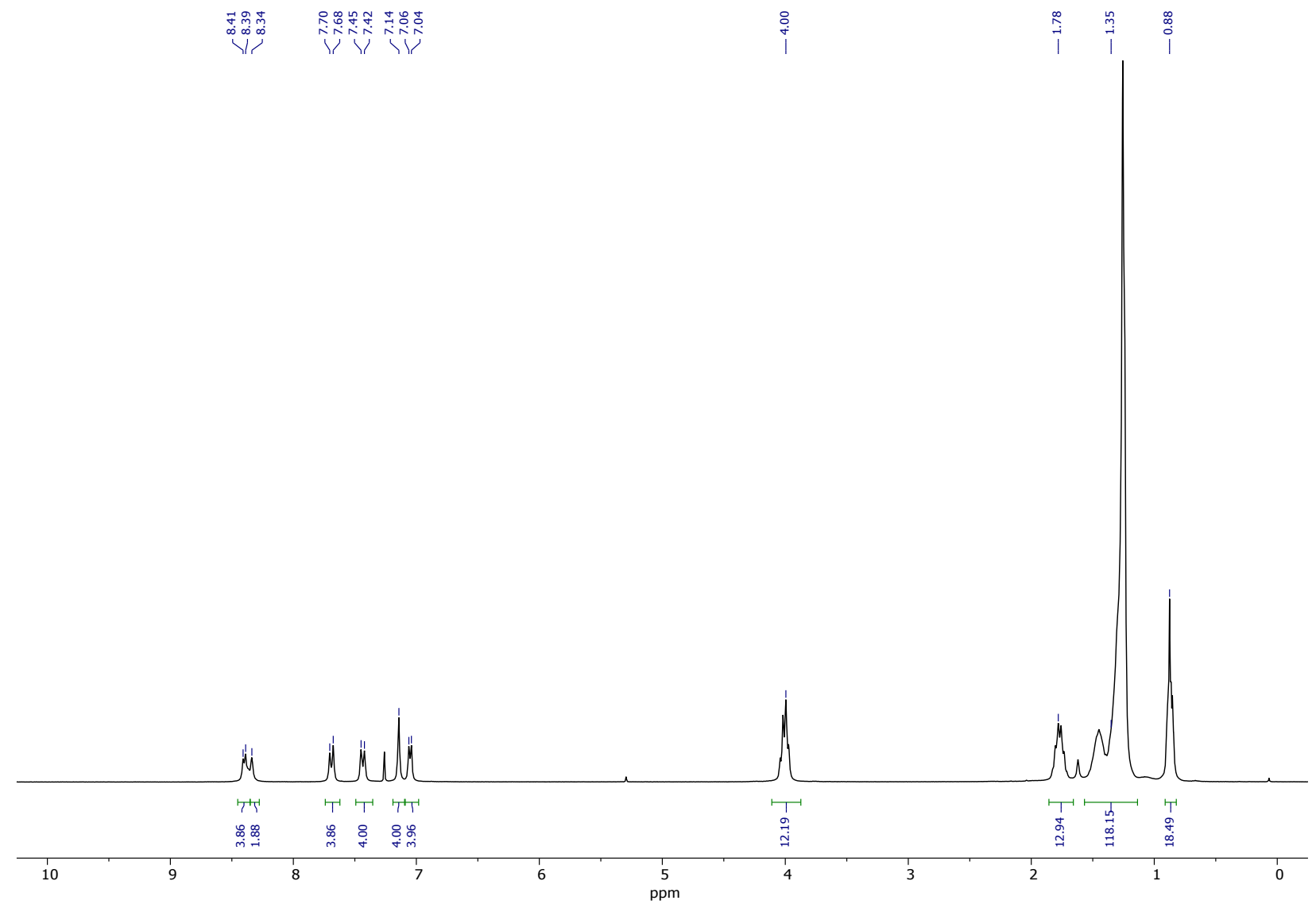

Figure S51. ${ }^{1} \mathrm{H}$ NMR $\left(\mathrm{CDCl}_{3}, 300 \mathrm{MHz}\right)$ spectrum of complex 2. 


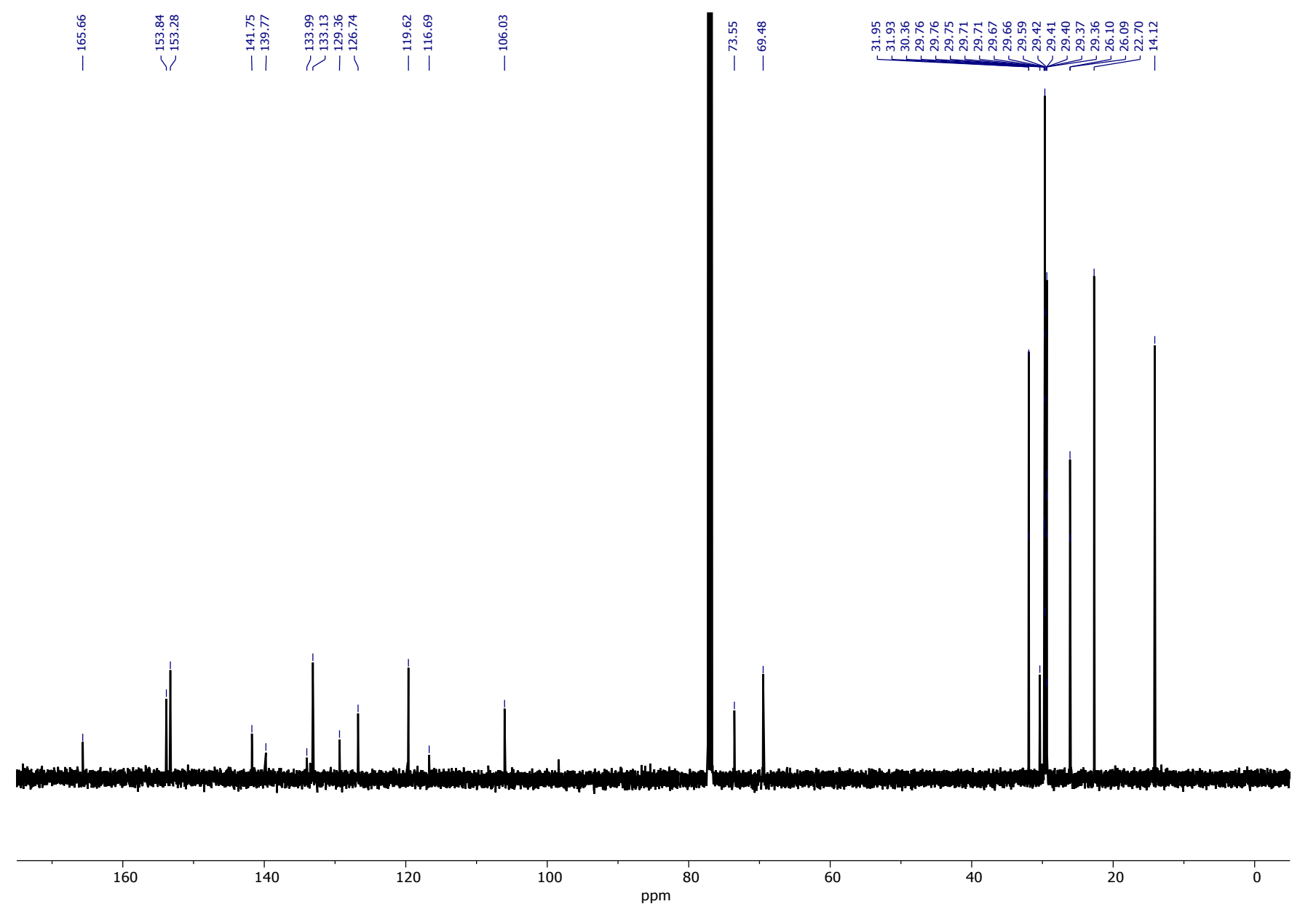

Figure S52. ${ }^{13} \mathrm{C}$ NMR $\left(\mathrm{CDCl}_{3}, 126 \mathrm{MHz}\right)$ spectrum of complex 2 .

\section{Characterization of complex 3}

${ }^{1} \mathrm{H}_{\mathrm{NMR}}\left(\mathrm{CDCl}_{3}, 500 \mathrm{MHz}\right) \delta(\mathrm{ppm})=8.85\left(\mathrm{AA}^{\prime} \mathrm{XX} \mathrm{X}^{\prime}, 4 \mathrm{H}, \mathrm{H}_{1}\right), 7.84\left(\mathrm{~s}, 2 \mathrm{H}, \mathrm{H}_{5}\right), 7.70\left(\mathrm{~m}, 4 \mathrm{H}, \mathrm{H}_{4}\right), 7.56\left(\mathrm{~m}, 4 \mathrm{H}, \mathrm{H}_{3}\right), 7.25\left(\mathrm{AA}^{\prime} \mathrm{XX}^{\prime}\right.$, $\left.4 \mathrm{H}, \mathrm{H}_{2}\right), 7.06\left(\mathrm{~s}, 4 \mathrm{H}, \mathrm{H}_{6}\right), 4.04\left(\mathrm{~m}, 12 \mathrm{H}, \mathrm{OCH}_{2}\right) 1.80\left(\mathrm{~m}, 12 \mathrm{H}, \mathrm{C}^{2} \mathrm{H}_{2}\right), 1.48\left(\mathrm{~m}, 12 \mathrm{H}, \mathrm{C}^{3} \mathrm{H}_{2}\right) 1.30\left(\mathrm{~m}, 96 \mathrm{H}, \mathrm{C}^{4} \mathrm{H}_{2}-\mathrm{C}^{11} \mathrm{H}_{2}\right), 0.88(\mathrm{t}$, $J=6.9 \mathrm{~Hz}, 18 \mathrm{H})$.

${ }^{13} \mathrm{C}$ NMR $\left(126 \mathrm{MHz}, \mathrm{CDCl}_{3}\right) \delta(\mathrm{ppm})=165.76,155.17,153.50,142.09,126.97,119.88,116.97,106.14,98.32,69.73,32.10$, $32.08,30.49,29.91,29.90,29.89,29.86,29.82,29.80,29.73,29.56,29.55,29.52,26.24,22.85,14.27,1.18$.

HR-MS (ESI-Orbitrap, $\mathrm{MeOH} / \mathrm{CHCl}_{3}$ ): found:

calculated for $\mathrm{C}_{112} \mathrm{H}_{172} \mathrm{~N}_{4} \mathrm{O}_{8} \mathrm{I}_{2} \mathrm{PtNa}_{2}{ }^{2+}[\mathrm{M}+2 \mathrm{Na}]^{2+}$ : $\quad$ 1098.53624, 1098.53620 


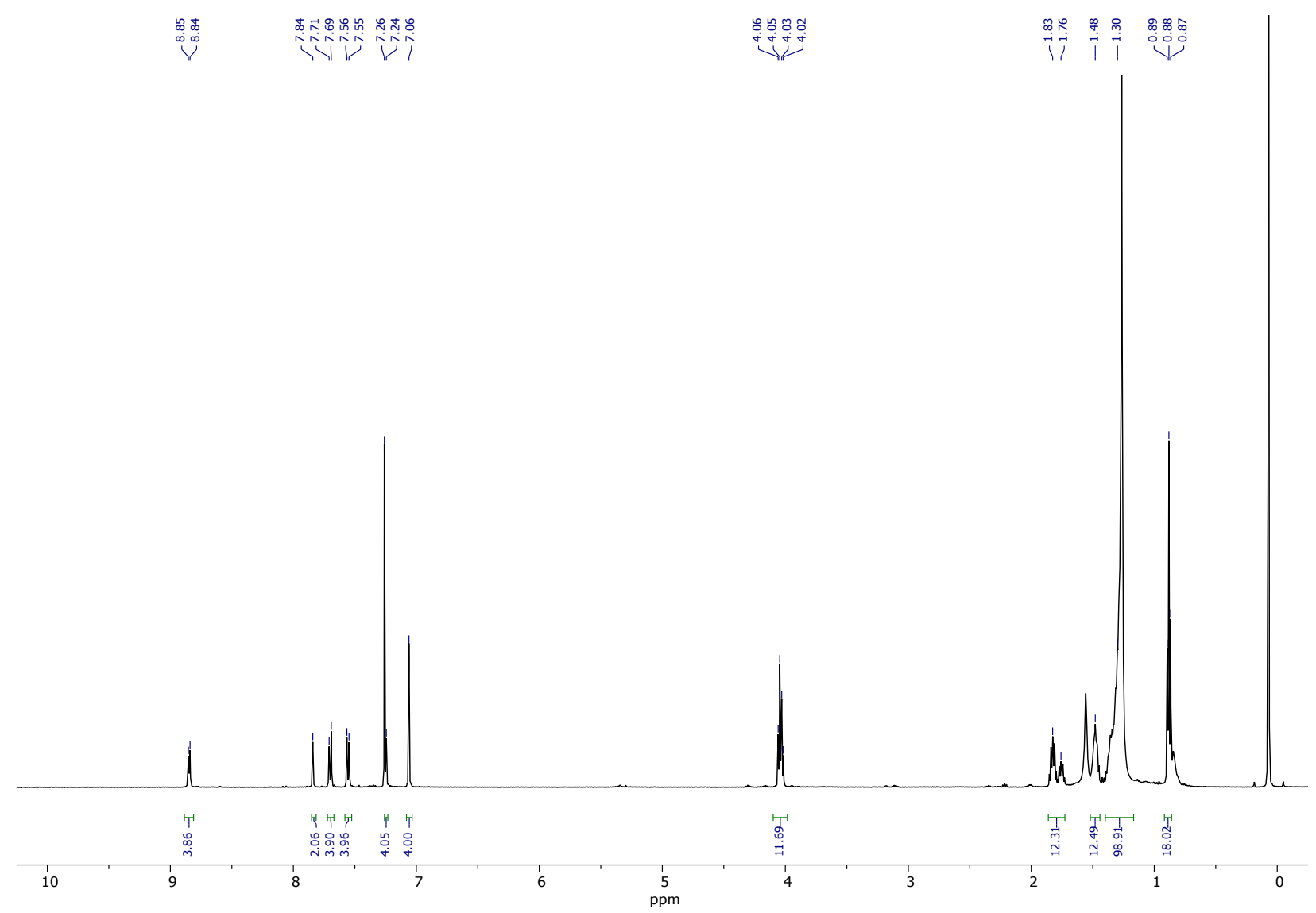

Figure S53. ${ }^{1} \mathrm{H}$ NMR $\left(\mathrm{CDCl}_{3}, 500 \mathrm{MHz}\right)$ spectrum of complex 3.

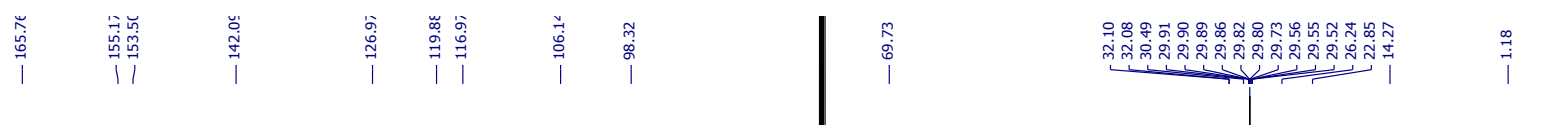
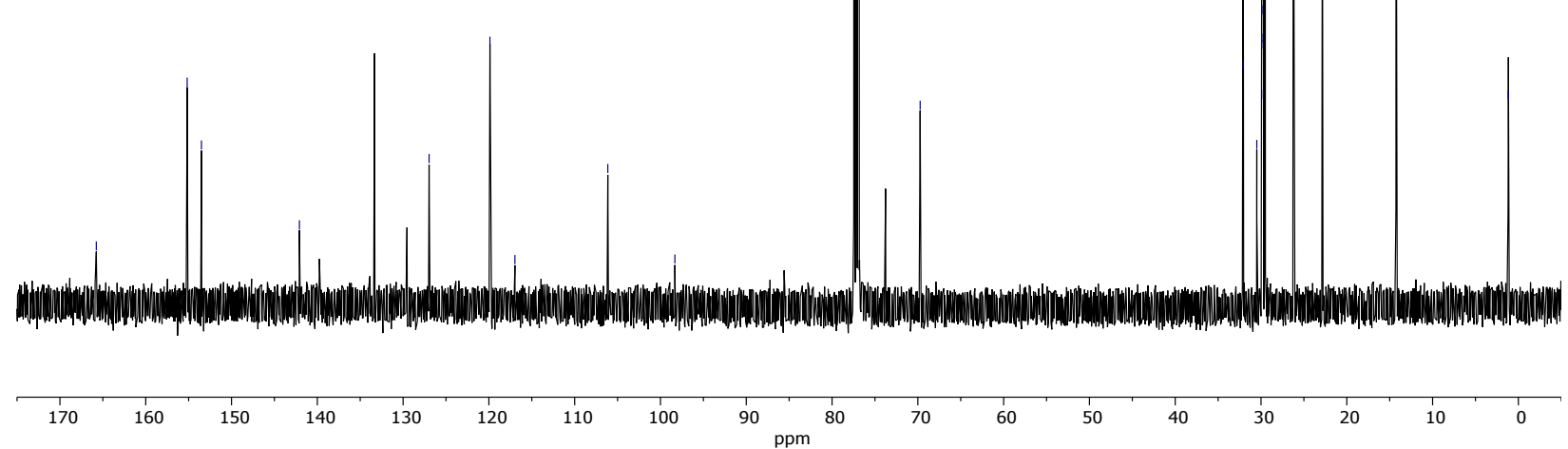

Figure S54. ${ }^{13} \mathrm{C}$ NMR $\left(\mathrm{CDCl}_{3}, 126 \mathrm{MHz}\right)$ spectrum of complex 3. 


\section{References}

(1) Korevaar, P. A.; Schaefer, C.; de Greef, Tom F. A.; Meijer, E. W. Controlling Chemical Self-Assembly by Solvent-Dependent Dynamics. J. Am. Chem. Soc. 2012, 134, 13482-13491.

(2) Goldstein, R. F.; Stryer, L. Cooperative polymerization reactions. Analytical approximations, numerical examples, and experimental strategy. Biophys. J. 1986, 50, 583-599.

(3) Langenstroer, A.; Kartha, K. K.; Dorca, Y.; Droste, J.; Stepanenko, V.; Rodrigo Q., A.; Hansen, M. R.; Sánchez, L.; Fernández, G. Unravelling Concomitant Packing Polymorphism in Supramolecular Polymers. J. Am. Chem. Soc. 2019, 141, 5192-5200.

(4) Matern, J.; Kartha, K. K.; Sánchez, L.; Fernández, G. Consequences of hidden kinetic pathways on supramolecular polymerization. Chem. Sci. 2020, 11, 6780-6788.

(5) Stewart, J. J. P. Optimization of parameters for semiempirical methods VI: more modifications to the NDDO approximations and reoptimization of parameters. J. Mol. Model. 2013, 19, 1-32.

(6) James J. P. Stewart. MOPAC2016; Stewart Computational Chemistry. http://openmopac.net/.

(7) Shao, Y.; Gan, Z.; Epifanovsky, E.; Gilbert, A. T.B.; Wormit, M.; Kussmann, J.; Lange, A. W.; Behn, A.; Deng, J.; Feng, X.; Ghosh, D.; Goldey, M.; Horn, P. R.; Jacobson, L. D.; Kaliman, I.; Khaliullin, R. Z.; Kuś, T.; Landau, A.; Liu, J.; Proynov, E. I.; Rhee, Y. M.; Richard, R. M.; Rohrdanz, M. A.; Steele, R. P.; Sundstrom, E. J.; Woodcock, H. L.; Zimmerman, P. M.; Zuev, D.; Albrecht, B.; Alguire, E.; Austin, B.; Beran, G. J. O.; Bernard, Y. A.; Berquist, E.; Brandhorst, K.; Bravaya, K. B.; Brown, S. T.; Casanova, D.; Chang, C.-M.; Chen, Y.; Chien, S. H.; Closser, K. D.; Crittenden, D. L.; Diedenhofen, M.; DiStasio, R. A.; Do, H.; Dutoi, A. D.; Edgar, R. G.; Fatehi, S.; Fusti-Molnar, L.; Ghysels, A.; Golubeva-Zadorozhnaya, A.; Gomes, J.; Hanson-Heine, M. W.D.; Harbach, P. H.P.; Hauser, A. W.; Hohenstein, E. G.; Holden, Z. C.; Jagau, T.-C.; Ji, H.; Kaduk, B.; Khistyaev, K.; Kim, J.; Kim, J.; King, R. A.; Klunzinger, P.; Kosenkov, D.; Kowalczyk, T.; Krauter, C. M.; Lao, K. U.; Laurent, A. D.; Lawler, K. V.; Levchenko, S. V.; Lin, C. Y.; Liu, F.; Livshits, E.; Lochan, R. C.; Luenser, A.; Manohar, P.; Manzer, S. F.; Mao, S.-P.; Mardirossian, N.; Marenich, A. V.; Maurer, S. A.; Mayhall, N. J.; Neuscamman, E.; Oana, C. M.; Olivares-Amaya, R.; O’Neill, D. P.; Parkhill, J. A.; Perrine, T. M.; Peverati, R.; Prociuk, A.; Rehn, D. R.; Rosta, E.; Russ, N. J.; Sharada, S. M.; Sharma, S.; Small, D. W.; Sodt, A.; Stein, T.; Stück, D.; Su, Y.-C.; Thom, A. J.W.; Tsuchimochi, T.; Vanovschi, V.; Vogt, L.; Vydrov, O.; Wang, T.; Watson, M. A.; Wenzel, J.; White, A.; Williams, C. F.; Yang, J.; Yeganeh, S.; Yost, S. R.; You, Z.-Q.; Zhang, I. Y.; Zhang, X.; Zhao, Y.; Brooks, B. R.; Chan, G. K.L.; Chipman, D. M.; Cramer, C. J.; Goddard, W. A.; Gordon, M. S.; Hehre, W. J.; Klamt, A.; Schaefer, H. F.; Schmidt, M. W.; Sherrill, C. D.; Truhlar, D. G.; Warshel, A.; Xu, X.; Aspuru-Guzik, A.; Baer, R.; Bell, A. T.; Besley, N. A.; Chai, J.-D.; Dreuw, A.; Dunietz, B. D.; Furlani, T. R.; Gwaltney, S. R.; Hsu, C.-P.; Jung, Y.; Kong, J.; Lambrecht, D. S.; Liang, W.; Ochsenfeld, C.; Rassolov, V. A.; Slipchenko, L. V.; Subotnik, J. E.; van Voorhis, T.; Herbert, J. M.; Krylov, A. I.; Gill, P. M.W.; Head-Gordon, M. Advances in molecular quantum chemistry contained in the Q-Chem 4 program package. Mol. Phys. 2015, 113, 184-215.

(8) Lee, C.; Yang, W.; Parr, R. G. Development of the Colle-Salvetti correlation-energy formula into a functional of the electron density. Phys. Rev. B 1988, 37, 785-789.

(9) Becke, A. D. Density-functional thermochemistry. III. The role of exact exchange. J. Chem. Phys. 1993, 98, 5648-5652.

(10) Coelho, J. P.; Mayoral, M. J.; Camacho, L.; Martín-Romero, M. T.; Tardajos, G.; López-Montero, I.; Sanz, E.; Ávila-Brande, D.; GinerCasares, J. J.; Fernández, G.; Guerrero-Martínez, A. Mechanosensitive Gold Colloidal Membranes Mediated by Supramolecular Interfacial SelfAssembly. J. Am. Chem. Soc. 2017, 139, 1120-1128.

(11) Gunawardana, C. A.; Sinha, A. S.; Desper, J.; Đaković, M.; Aakeröy, C. B. Supramolecular Chemistry of Some Metal Acetylacetonates with Auxiliary Pyridyl Sites. Cryst. Growth Des. 2018, 18, 6936-6945.

(12) Usón, R.; Forniés, J.; Tomás, M.; Ara, I. Synthesis and reactivity of trinuclear complexes of platinum containing the single bridging ligand $\mathrm{SC}_{4} \mathrm{H}_{8}$. Molecular structure of $\left[\mathrm{NBu}_{4}\right]_{2}$ [trans- $\left.\mathrm{PtCl}_{2}\left\{\left(\mu-\mathrm{SC}_{4} \mathrm{H}_{8}\right) \mathrm{Pt}\left(\mathrm{C}_{6} \mathrm{~F}_{5}\right)_{3}\right\}_{2}\right]$. J. Chem. Soc., Dalton Trans. 1989, $1011-1016$.

(13) Yahav, A.; Goldberg, I.; Vigalok, A. Difluoro Complexes of Platinum(II) and -(IV) with Monodentate Phosphine Ligands: An Exceptional Stability of $\mathrm{d}^{6}$ Octahedral Organometallic Fluorides. Inorg. Chem. 2005, 44, 1547-1553.

(14) Grushin, V. V.; Marshall, W. J. trans-Difluoro Complexes of Palladium(II). J. Am. Chem. Soc. 2009, 131, 918-919.

(15) Yahav, A.; Goldberg, I.; Vigalok, A. Synthesis of the Elusive $\left(\mathrm{R}_{3} \mathrm{P}\right)_{2} \mathrm{MF}_{2}(\mathrm{M}=\mathrm{Pd}, \mathrm{Pt})$ Complexes. J. Am. Chem. Soc. 2003, 125, 1363413635.

(16) Grushin, V. V. Palladium Fluoride Complexes: One More Step toward Metal-Mediated C-F Bond Formation. Chem. Eur. J. 2002, 8, 1006-1014.

(17) Murphy, E. F.; Murugavel, R.; Roesky, H. W. Organometallic Fluorides: Compounds Containing Carbon-Metal-Fluorine Fragments of d-Block Metals. Chem. Rev. 1997, 97, 3425-3468.

(18) Doherty, N. M.; Hoffmann, N. W. Transition-metal fluoro compounds containing carbonyl, phosphine, arsine, and stibine ligands. Chem. Rev. 1991, 91, 553-573.

(19) Mezzetti, A.; Becker, C. Swimming against the Stream? A Discussion of the Bonding in $\mathrm{d}^{6}$ and $\mathrm{d}^{8}$ Fluoro Complexes and Its Consequences for Catalytic Applications. Helv. Chim. Acta 2002, 85, 2686-2703. 